Review

\title{
Diketopyrrolopyrrole Fluorescent Probes, Photophysical and Biological Applications
}

\author{
Muhammad Aminu Auwalu (D) and Shanshan Cheng*(D) \\ Department of Chemistry, Tianjin Key Laboratory of Molecular Optoelectronic Sciences, School of Science, Tianjin \\ University, Tianjin 300072, China; maa@tju.edu.cn \\ * Correspondence: chengss@tju.edu.cn; Tel.: +86-0228-3613-171
}

check for

updates

Citation: Auwalu, M.A.; Cheng, S. Diketopyrrolopyrrole Fluorescent Probes, Photophysical and Biological Applications. Chemosensors 2021, 9, 44. https://doi.org/10.3390/ chemosensors 9030044

Academic Editor: Bolze Frederic

Received: 17 January 2021

Accepted: 22 February 2021

Published: 26 February 2021

Publisher's Note: MDPI stays neutral with regard to jurisdictional claims in published maps and institutional affiliations.

Copyright: (C) 2021 by the authors. Licensee MDPI, Basel, Switzerland. This article is an open access article distributed under the terms and conditions of the Creative Commons Attribution (CC BY) license (https:// creativecommons.org/licenses/by/ $4.0 /)$.

\begin{abstract}
Biological applications of fluorescent probes are rapidly increasing in the supramolecular chemistry research field. Several organic dyes are being utilized currently in developing and advancing this attractive research area, of which diketopyrrolopyrrole (DPP) organic dyes show an exceptional photophysical features (high-fluorescence quantum yield (FQY), good photochemical and thermal stability) that are essential properties for biological applications. Great efforts have been made in recent years towards developing novel fluorescent DPPs by different chemists for such applications, and some positive results have been reported. As a result, this review article gives an account of the progress that has so far been made very recently, mainly within the last decade, in that we selectively focus on and discuss more from 2015 to present on some recent scholarly achievements of fluorescent DPPs: quantum yield, aggregation-induced emission (AIE), solid-state emission, bio-imaging, cancer/tumor therapy, mitochondria staining and some polymeric fluorescent DPPs. Finally, this review article highlights researchers working on luminescent DPPs and the future prospects in some key areas towards designing DPP-based fluorescent probes in order to boost their photophysical and biological applications more effectively.
\end{abstract}

Keywords: diketopyrrolopyrrole; fluorescence; quantum yield; aggregation-induced emission; solidstate emission; bio-imaging; cancer therapy

\section{Introduction}

Fluorescent probes (FPs) are materials possessing fluorescence properties, like fluorescence time, wavelength and emission intensity, changes resulting from specific interactions with target species for sensing and visualizing of some biological molecules as a result of their progressiveness, noninvasiveness and high sensitivity with spatial resolution, hence gaining considerable research interests [1-5]. Over the years, organic dyes (ODs) have been used in the fluorescence research field to develop probes for different kinds of applications, such as optoelectronics [6], organic electronics light-emitting devices like OLEDs, solar cells and OFETs [7-11], with detection of essential biological components, bio-imaging and even therapy of cancer/tumor cells [12-15]. Such utilized novel FPs are mostly from coumarin, rhodamine, fluorescein, cyanine, boron-dipyrromethane (BODIPY) and diketopyrrolopyrrole (DPP). This is because these fluorogenic core units possess outstanding photoluminescence (PL) properties [16].

Coumarin dyes absorb and emit in the visible region high-energy wavelength (blueshift) [17], thus limiting their biological applications. High-fluorescence quantum yields (FQYs) and good photostability are characteristic features of fluorescein $[18,19]$ and rhodamine [20] dyes, but such dyes have a poor role to play biologically, as their absorption/emission maxima takes place mostly below $600 \mathrm{~nm}$ [16], hence limiting their biological applications. Undoubtedly, cyanine dyes biologically have a good role to play [21-25]; however, their moderately low photostability hindered their biological applications, as well in relation to fluorescein and rhodamine dyes. Derivatives of BODIPY possessed excellent 
absorption/emission maxima along with high molar absorptivity, thus attracting research interest, too $[26,27]$. BODIPY and DPP dyes both possessed features suitably useful in supramolecular chemistry (SC) the (high FQY, excellent absorption/emission and very good photostability).

However, during the past decade, DPP dyes have auspiciously attracted considerable research attention in this research field, especially in biological systems. This is as a result of fluorescent DPPs' inherent absorption and emission properties to have predominantly moves red-shift in an electromagnetic spectrum. Essentially important is the fact the typical DPP skeleton without any optimization fluoresce strongly above $500 \mathrm{~nm}$ (greenyellow) [28], and proper functionalization moves it easily to red-shift to the near-infrared region (NIR) high wavelength (low-energy) with improved absorption/emission above $600 \mathrm{~nm}$, and even far red to (800-900) nm, hence establishing strongly optimized DPP fluorophores with narrow NIR and far-red absorption at lower energy. This is because fluorophores fluorescing at higher wavelengths are essentially important biologically, as their cells and water absorption, autofluorescence and light scattering are greatly reduced, thus facilitating light penetrability deeply and hence boosting their therapeutic/diagnostic functions more effectively. Moreover, DPP dyes are deeply bright red dyeing pigments, and, accordingly, now their molar absorptivity is relatively high, $\sim 76,000 \mathrm{M}^{-1} \mathrm{~cm}^{-1}$, close to that of BODIPY, displaying $~ 80,000 \mathrm{M}^{-1} \mathrm{~cm}^{-1}$. These mentioned ODs' photophysical properties are briefly summarized in Table 1 , below.

Table 1. Compared photophysical features of diketopyrrolopyrrole (DPP) with mostly used organic dyes.

\begin{tabular}{ccccccc}
\hline Organic Dyes & Abs./Em. (nm) & $\begin{array}{c}\text { Abs./Em. Max } \\
\mathbf{( n m )}\end{array}$ & Photostability & $\begin{array}{c}\text { Molar Absorptivity } \\
\mathbf{( M}^{-\mathbf{1}} \mathbf{~} \mathbf{m}^{-\mathbf{1}} \mathbf{)}\end{array}$ & FQY & Reference \\
\hline DPP & $400-500 / 500-600$ & $500>/ 650>$ & Extremely High & 76,000 & $0.4-0.9$ & This work \\
Rhodamine & $470-528 / 510-610$ & $530 / 621$ & Good & 116,000 & 0.95 & {$[16]$} \\
Fluorescein & $400-550 / 460-700$ & $494 / 512$ & High & 80,000 & 0.92 & {$[16]$} \\
Cyanine & $400-800 / 500-800$ & $473 / 767$ & High & 100,000 & $0.12-0.67$ & {$[16]$} \\
Coumarin & $320-520 / 450-590$ & $420 / 460$ & Moderately & 20,000 & 0.78 & {$[16]$} \\
BODIPY & $500-645 / 506-760$ & $503 / 512$ & Extremely High & 80,000 & 0.92 & {$[16]$} \\
\hline
\end{tabular}

Abs, absorption; Em, emission; DPP, diketopyrropyrrole; BODIPY, boron-dipyrromethane; FQY, high-fluorescence quantum yield.

DPP-based FPs has a very high FQY, good light and are thermally stable (excellently photostable), thus serving as unique building blocks for various applications primarily for field-effect transistors [29-31] and photovoltaic cells [32-34] due to their substantial semiconducting properties. Different DPP-based FPs are being reported from the literature for numerous applications which can be categorically classified as; those for analytes (ions, ROS, thiols, $\mathrm{pH}, \mathrm{CO}_{2}$ and $\mathrm{H}_{2}$ ) [35-37] molecular/and or bio-imaging probes [38], the near-infrared (NIR) dyes [39] and even luminescent polymer probes [40], etc. However, despite such developmental progress for this class of high performing DPP-based FPs, yet their potentials to detect essentially important biological molecules is still infantile. Considering the DPP's exceptional photophysical features, recently efforts are being made towards advancing its FPs and investigating their biosensing capability [41-43] in order to lessen such challenges and difficulties precisely for SC using various strategies owing to the low-energy of its optimized fluorophore essentially useful, especially in solutions and membrane-like systems.

These peculiar properties have made DPP fluorophore currently attracting significant research attention, as many research papers are reported. Thus, this review article specifically focuses on studying FPs in ODs, precisely DPP, because of its distinct brilliant red-dyeing pigment, considering its positive applications in SC, as compared to the most commonly used ODs, as briefly discussed in this section. 


\subsection{Overview of the Typical DPP Fluorophore}

The DPP skeletal unit possesses actively different functionalities (Figure 1), the bis(lactam) unit having functional groups of alkenes and carbonyl (ketones) coupled with secondary amidic hydrogen $(\mathrm{N}-\mathrm{H})$, which undergoes different functionalization to generate various derivatives of the DPP materials. The DPP core unit is obviously known for its high electron affinity, thus making it suitable for both electrophilic and nucleophilic substitution reaction. DPPs have been recently attracting considerable research attention, simply due to their outstanding and distinctive photophysical features, as summarized in Table 1, above. Therefore, they are unique semiconductor building blocks for both hybrid and purely organic materials, with remarkable semiconducting properties primarily for Organic Field-Effect Transistors (OFETs), photovoltaic devices and other relevant organic optoelectronics application. The typical DPP fluorophore absorbs and fluoresces strongly, exceeding $500 \mathrm{~nm}$ (green-yellow emission) without modification, and proper optimization moves it easily red-shift; thus, such modified low-energy fluorophores are useful for biosensing and bio-imaging applications, as briefly introduced and discussed. These features have caused DPP to attract considerable research attention for the past few years and present. Such FPs, with their several applications, were recently reviewed by References $[3,4,9,44]$, for further readings.

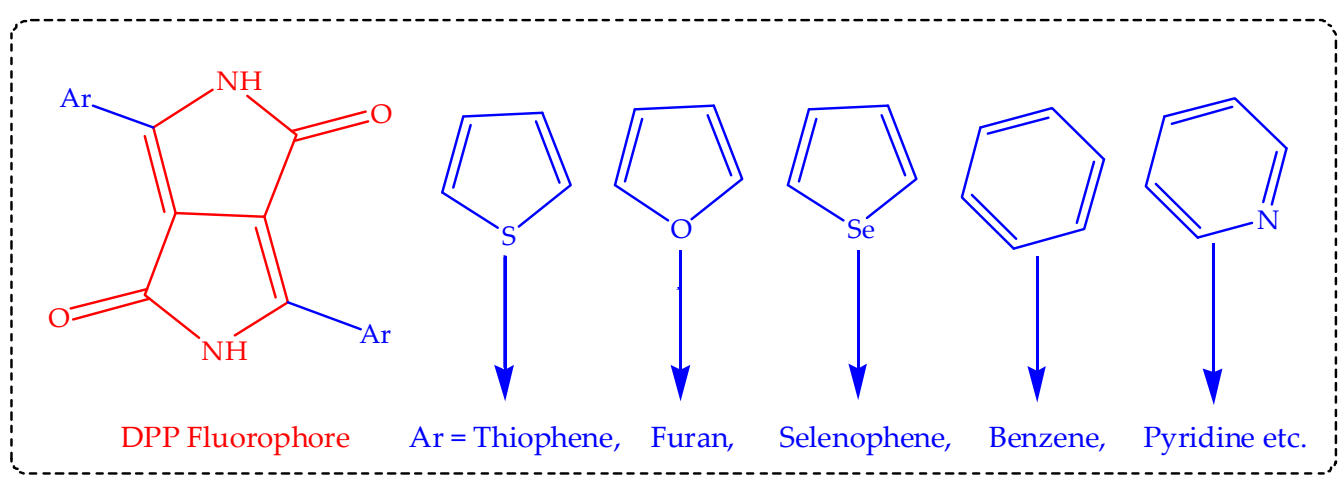

Figure 1. The typical DPP fluorophore's skeleton structure with some representative various donor units.

\subsection{Functionalization of the DPP-Core Unit}

Researchers working with this commonly used OD of DPP fluorophore generally adopt two strategic approaches in modifying the DPP core (Figure 2) to develop new derivative with desirable features in mind for different optoelectronics application. These two approaches are (1) functionalizing the DPP core with redox active metals at amide hydrogen $(\mathrm{N}-\mathrm{H})$ and (2) functionalization through side-chain engineering at either the $(\mathrm{N}-$ $\mathrm{H})$ or the carbonyl center $(\mathrm{C}=\mathrm{O})$ of the of the typical DPP core unit. Moreover, when DPP is optimized at the amide position, it mostly generates new derivatives with improved FQY, and when the core unit is functionalized at the $3 / 6$ position, the resulting DPP derivatives displayed improved absorption/emission to NIR region of an electromagnetic spectrum. These modification strategies are all briefly discussed in the following subsections below. 


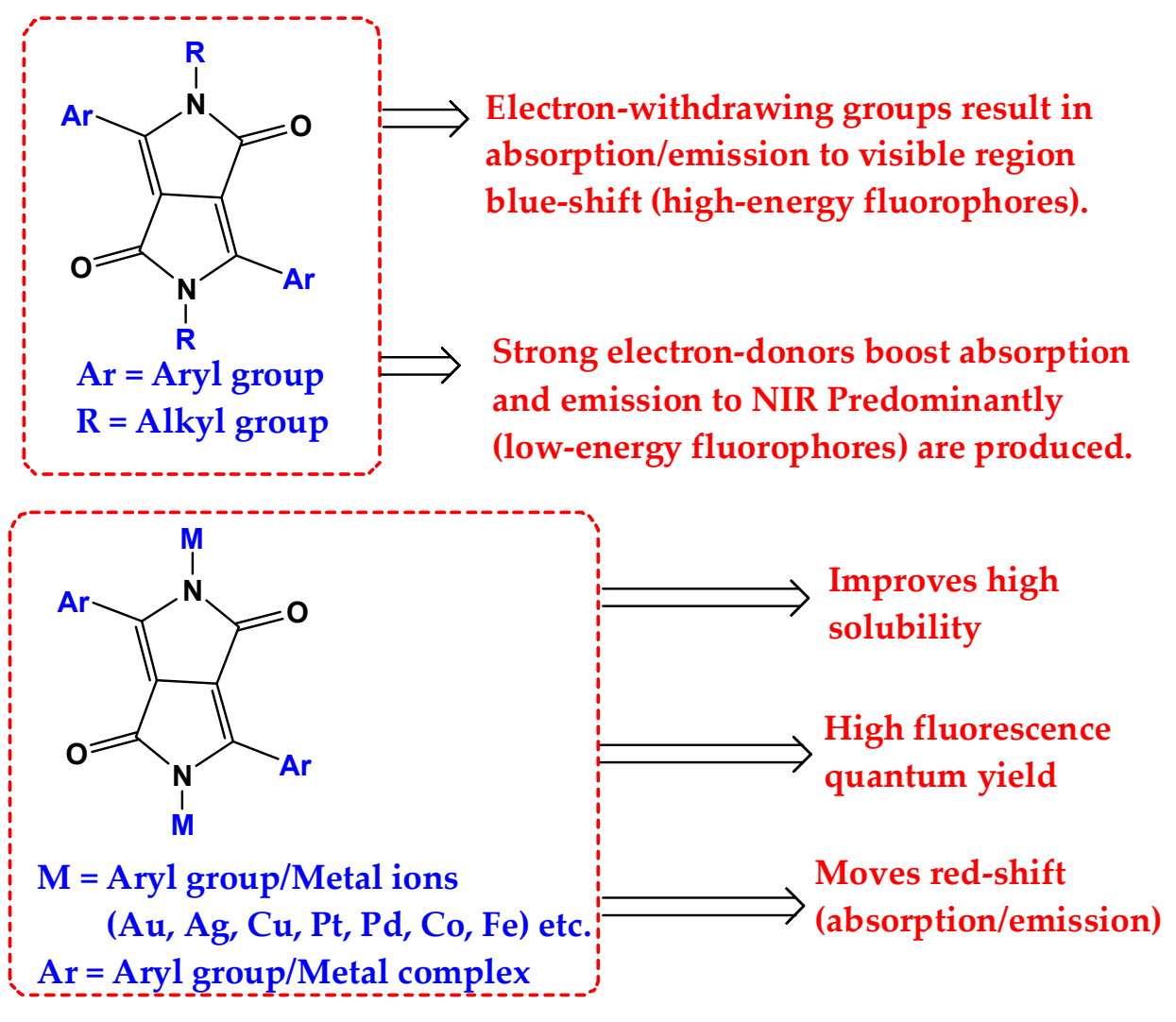

Figure 2. The general DPP core modification strategies using different electrondonating/withdrawing groups at lactam nitrogen and 3/6 positions.

\subsubsection{Side-Chain Engineered DPP Materials}

Side-chain engineering (SCE) is proven effective in organic chemistry in optimizing different materials, purposely to fine-tune the materials' intrinsic properties for a particular application [45-48]. Such substituent groups are principally employed as solubilizing groups; however, their potential is far beyond that. In a nutshell, through side-chain engineering of small molecules and conjugated organic materials, their chemical properties can be enhanced to attain certain features that are suitable for specific application. For both small and organic conjugated molecules (polymers), SCE is being used to primarily boost the materials electronic (absorption/emission), optoelectronics properties and even sensing functionality (detection of different analytes) in supramolecular chemistry significantly.

Scientifically, small molecules are easy to purify; as is obviously known, the purity of a material is of crucial importance to device performance, and the $\pi-\pi$ overlapping of small organic molecules ensures the efficient flow of charges. Additionally, small molecules are easy to form single-crystal or 2D-crystal thin-films. However, for polymers, the molecular weight and side-chain of polymers are varied, because they are more difficult to purify and do not guarantee good charge transport like small molecules; therefore, they often result in having lower mobility compared to organic small molecules, generally. Thus, in small molecules, there exist free mobile electrons that can resonate within the molecular framework due the relatively high materials purity, which provides efficient flow of charges. Furthermore, in small molecules, the band gap is relatively small compared to conjugated organic materials like polymers, due to poor purity, which lessens the efficient flow of charges within the polymer backbone, thus affecting the luminescent properties of emissive polymers like polymeric fluorescent materials. Therefore, the ease for purification and high purity of small organic molecules makes them appealing, and they attract much attention in developing new molecules of DPP-based FPs, and these are reasons why a lot of FPs are from organic small molecules. Scheme 1 below shows some representative of the different 
small molecules FPs of DPP based on the SCE strategy. Moreover, these probes show absorption and emission in NIR (red-shift).
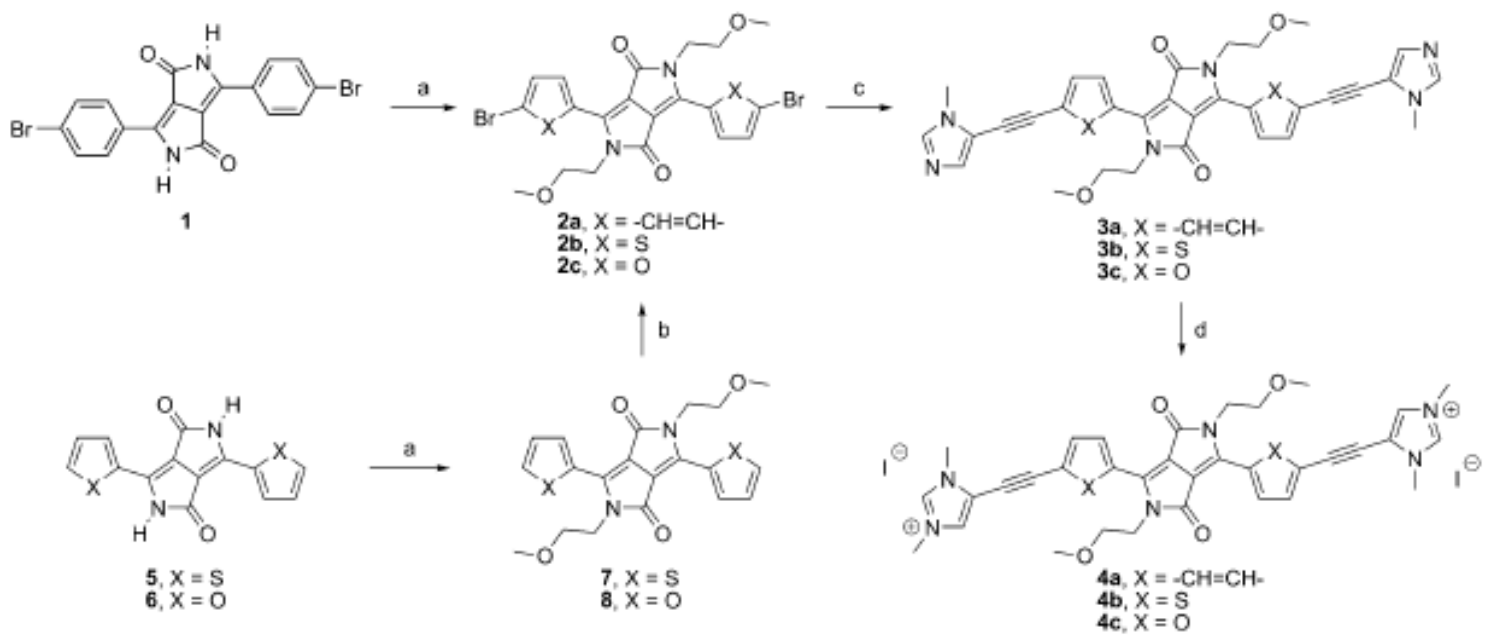

(a)

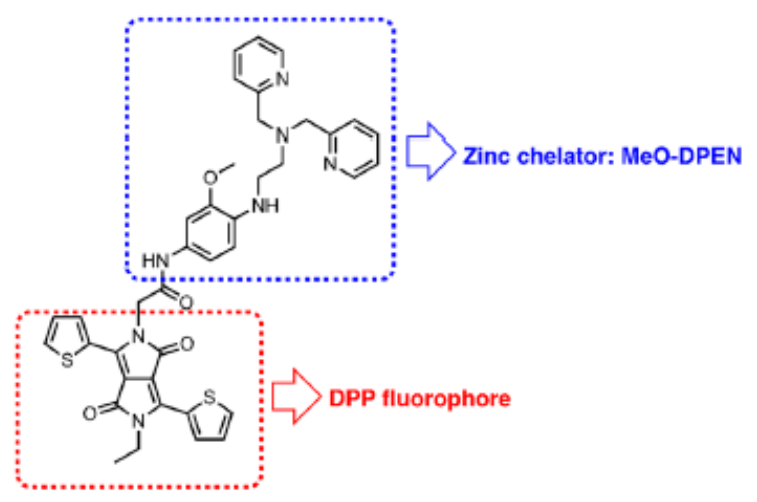

DPP-C2

(b)
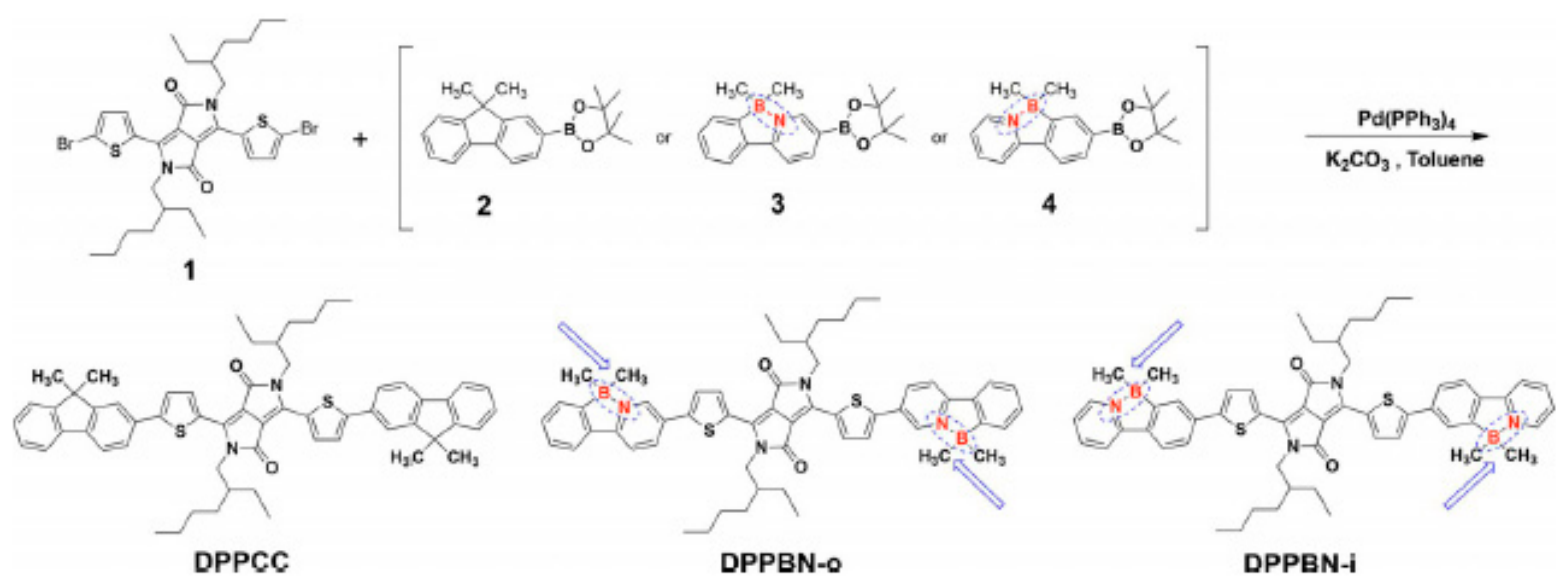

(c)

Scheme 1. Fluorescent DPP-based small molecules. (a) Produced cationic DPP probes. Copyright, 2015, reproduced with permission from Reference [41]. (b) DPP-C2 molecule structure. Copyright, 2019, reproduced with permission from Reference [49]. (c) Synthesized pathway of three structural DPPs composed of diverse side chains. Arrows directing the local dipole moments. Copyright, 2019, reproduced with permission from Reference [50]. 
Accordingly, these side-chains are being introduced onto the DPP core in two different ways, either by modifying the $\mathrm{N}-\mathrm{H}$ or $\mathrm{C}=\mathrm{O}$ functional groups. When optimized at the $\mathrm{N}-\mathrm{H}$ reactive center, the resulting new fluorophore mostly absorbs and emits in the visible region (blue-shift), though few of such derivatives absorb and emit at NIR (red-shift). On the other hand, when optimized at the carbonyl center ( $\mathrm{C}=\mathrm{O}$ ketone functional group), the resulting new material derivatives predominantly move red-shift and emit far above $600 \mathrm{~nm}$, giving near-infrared and far-infrared materials (low-energy). Scheme 2 illustrates some small molecule DPP FPs, along with representative DPP polymer FPs for various applications in solar cells, aggregation-induced emission (AIE), and solid-state emission, as discussed under the polymeric fluorescent DPP section. Again, these fluorophores absorb and emit in the NIR (red-shift).

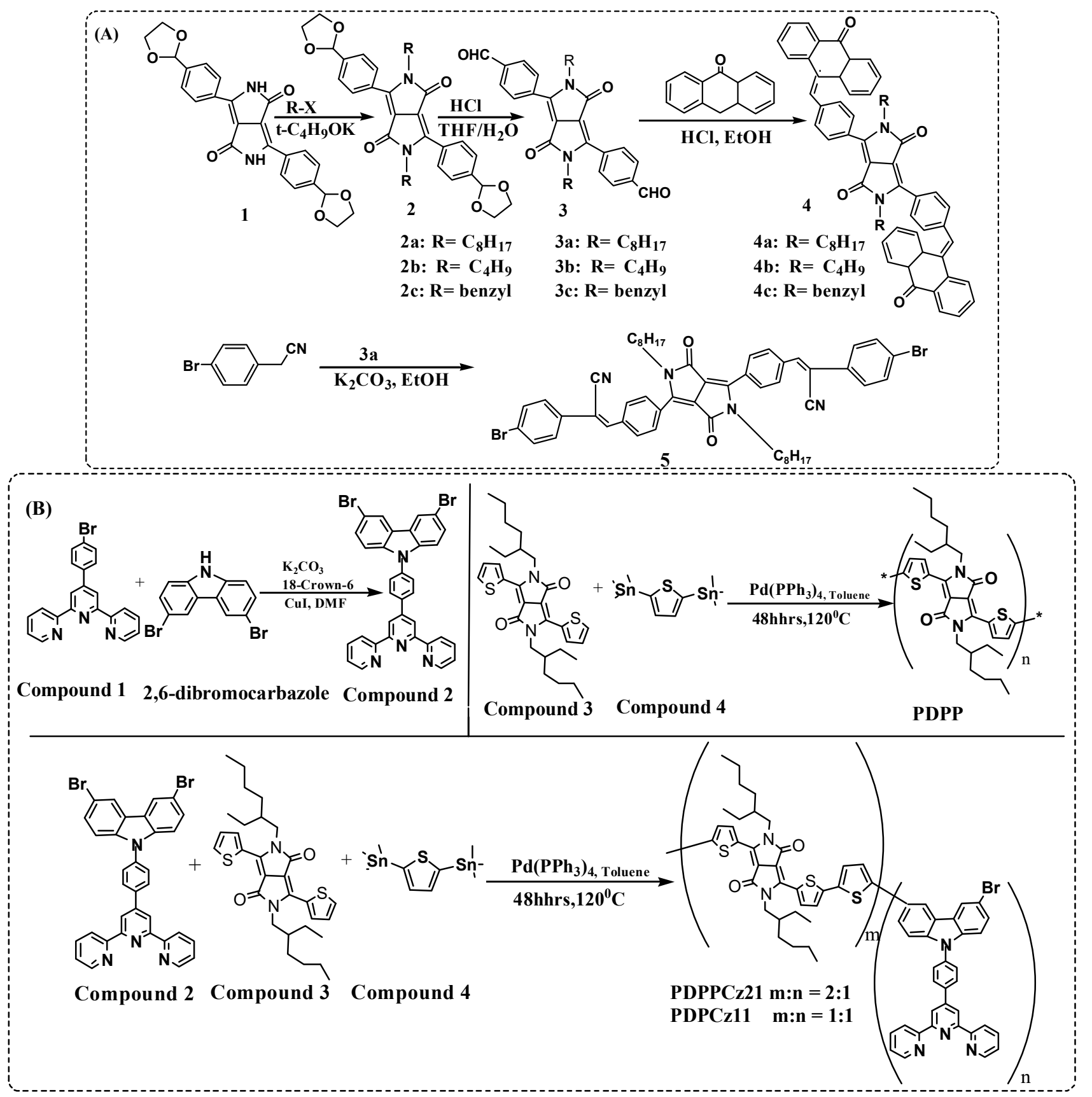

Scheme 2. Cont. 

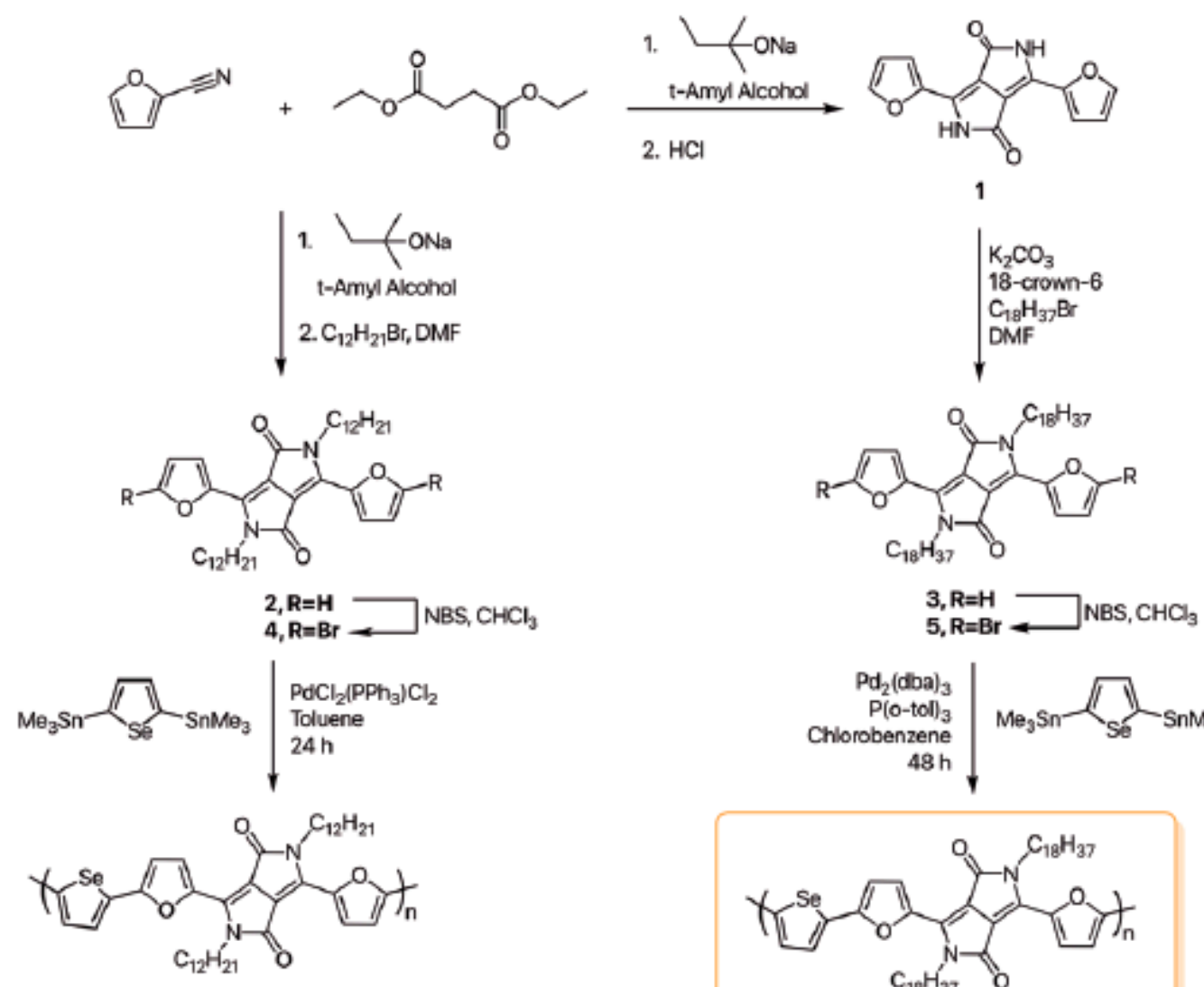

PFDPPSe-C12 (insoluble)
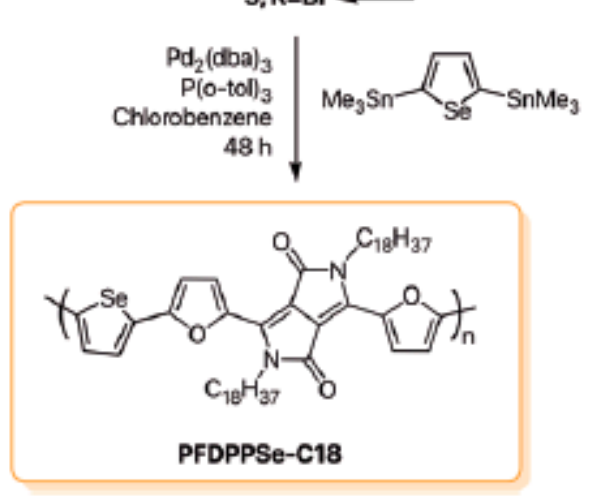

(C)

Scheme 2. (A) Synthesized small molecule DPPs 2-5 pathway. Copyright, 2011, reproduced with permission from Reference [51]. (B) DPP polythiophene polymers. Copyright, 2015, reproduced with permission from Reference [41]. (C) DPP furan-based selenophene polymers. Copyright, 2018, reproduced with permission from Reference [52].

\subsubsection{Metal-Functionalized DPP Materials}

Though DPPs are recently being considered as exceptional and distinct red OD pigments, they are insoluble in organic solvents. Their outstanding photophysical properties are influenced by the intermolecular H-bonding, which results in depressing the FQYs of DPP FPs, thus limiting their various applications in fluorescence research field. It follows that alkylating and arylating of the lactam nitrogen with diverse moieties significantly weakens the intermolecular H-bonding by improving the materials photophysical properties efficiently. It is crucially important to note that metallic ions interaction with receptor groups (electron-donating unit), significantly weakening the resulting materials' conjugation length, leading to a reduction in the potential of the donor unit to effectively donate electrons, thus making such materials absorb and emit in the blue-shifted region (high-energy wavelength). Alternatively, such interaction with acceptor unit moves the materials' absorption/emission to NIR (red-shifted), as a result of improvement in material's conjugation. Such a conjugation system enhancement raises the electron-withdrawing ability of the optimized acceptor component.

Accordingly, these desirable photophysical features can be further boosted by exploiting coordination chemistry [53], where the lactam nitrogen can be coordinated with metal ions to generate promising class of complexes with innovative properties, like higher solubility and higher FQY combined with bathochromic absorption for optoelectronic applications as demonstrated in (Figure 2). In 2000, Lorenz et al. synthesized a series 
of DPP complexes with gold $(\mathrm{Au})$, silver $(\mathrm{Ag})$ and copper $(\mathrm{Cu})$ elements. The authors prepared DPP dianions by reacting 2 moles hexamethyldisilazane sodium salt, which was additionally treated by using copper(I) chloride and silver(I) tetrafluoroborate triphenylphosphanes, and for gold, they used triphenylphosphane of gold(I) chloride. This synthetic approach reduces the likelihood of the formation of polymers, due to the steric effect of the bulkier triphenylphosphane substituent; the resulting complexes of $\mathrm{Cu}, \mathrm{Ag}$ and Au were made. The FQY of the starting DPP material was $50 \%$ and consequently, the metal complex of $\mathrm{Cu}$ displayed a $20 \%$ decline, which resulted from fluorescence quenching (FQ) induced by copper ion. Subsequently, the FQY was further decreased to $17 \%$ for the silver-complex derivative. However, gold complexes displayed a very strong unexpected increased to around $99 \%$, hence implying the effectiveness of sterically gold substituent in boosting the FQY of the DPP fluorophore [54]. Later, in 2002, the same authors further exploit the use of platinum $(\mathrm{Pt})$ and palladium $(\mathrm{Pd})$ metal complexes, and their resulting complexes with DPP showed negative results, and nearly 15\% FQY was observed for the platinum complex and almost indefinable discovered for palladium complexes [55].

This provides an insight that the vigilant choice of redox energetic metals possessing electron-donating capability undoubtedly offers competent metal complexes of DPP with desirable features applicable for non-linear optics, solar cells and photothermal cancer treatment.

\section{Fluorescence Quantum Yields (FQYs) and Emission Properties of DPP FPs}

The FQY of materials in other words quantum efficiency is solely the ability of the material's fluorophore to generate high output power (emitted photons divide by absorbed photons). Moreover, the extent with which the glowing material is able to emit the absorbed light (fluoresce), the better the FQY of that material. A 100\% FQY is obtained when the output power $=1$, this can only be achieved when the absorbed photons are equal to the emitted photons (1:1 ratio).

$$
\text { Quantum Yield }=\frac{\text { emitted photons }}{\text { absorbed photons }}
$$

Generally, most glowing materials possess a wider excitation and emission spectrum in nature; however, materials with evidently well-defined excitation and emission maxima are crucial and essentially more suitable in fluorescence microscopy like imagine. For this reason, DPP-based FPs are specifically selected owing to the DPPs obviously known very clear distinct absorption and emission wavelengths starting from 400 and $500 \mathrm{~nm}$, respectively [16], in an electromagnetic spectrum for almost all of its derivatives. Furthermore, such DPP FPs are rarely observed (especially in solid-state) among its several derivatives, according to the literature. Table 2, below, summarizes their different photophysical features.

As seen above, Table 2 summarizes the different photophysical features of some representative DPP FPs recently published, in that almost all of these probes displayed red-shifted absorption and emission in NIR, presenting appreciable FQY, molar absorptivity, Stokes's shift with good results for AIE, solid-state emission and biological applications. Precisely, their FQYs were quenched in some cases, which was likely due to solvent effect (polarity) or substituent effect incorporated with the DPP fluorophore being it electron-donating or electron-withdrawing groups. In essence, it is obviously known that donating groups result in generating the resulting fluorophores to predominantly absorb/emit in red-shift region; however, this depends on the donor group's interaction strength with the material's fluorophore through intramolecular charge transfer (ICT). However, withdrawing groups generally leads to developing fluorophores predominantly moves to blue-shift region low wavelength (high-energy) again, which relies on the withdrawing group strength and its interaction with the material's fluorophore under investigation via ICT. So in a nut shell, strong donating groups are essentially preferred when optimizing DPP fluorophore especially for biological applications, as such optimized fluorophores are significantly useful in SC, owing to their low-energy, which is very vital for fluorescence studies (bio-imaging, therapy, etc.), due to their deeper tissue penetration ability with nearly zero cytotoxicity. However, fluorescence 
turn-on/quenching events do happen particularly for applications in biological systems while interacting with target species; an example from Table 2 is briefly discussed, herein, under the molecular/bio-imaging probes section.

Table 2. Summarized the photophysical features of variously studied fluorescent DPPs.

\begin{tabular}{|c|c|c|c|c|c|c|c|}
\hline DPP FPs & Solvent & Abs./Em. (nm) & FQY (\%) & $\left(\mathrm{M}^{-1} \mathrm{~cm}^{-1}\right)$ & Stoke's Shift $\left(\mathrm{cm}^{-1}\right)$ & FL (ns) & Reference \\
\hline $7 \mathrm{e}\left(\mathrm{NMe}_{2}\right)$ & DCM & $684 / 753$ & 3 & 76,800 & 1322 & & {$[56]$} \\
\hline $7 \mathrm{a}(\mathrm{H})$ & DCM & $613 / 637$ & 36 & 44,500 & 600 & & [56] \\
\hline $7 \mathrm{~b}(\mathrm{Me})$ & DCM & $621 / 646$ & 32 & 54,700 & 610 & & [56] \\
\hline $7 \mathrm{f}(\mathrm{CN})$ & DCM & $622 / 656$ & 32 & 44,800 & 795 & & [56] \\
\hline 7c (SMe) & DCM & $627 / 664$ & 14 & 57,000 & 873 & & [56] \\
\hline 7d (OMe) & DCM & $631 / 662$ & 22 & 57,100 & 740 & & [56] \\
\hline PDPP-Hex & Toluene & $470 / 535$ & 85 & 12,600 & $0.32(\mathrm{eV})$ & 6.8 & [57] \\
\hline TDPP-Hex & Toluene & $510-525 / 565$ & 79 & 27,600 & $0.04(\mathrm{eV})$ & 6.2 & [57] \\
\hline SeDPP-Hex & Toluene & $550-562 / 580$ & 66 & 33,600 & $0.07(\mathrm{eV})$ & 5.5 & [57] \\
\hline FDPP-QAS & $\mathrm{H}_{2} \mathrm{O}$ & $534 / 588$ & 55 & & & & [58] \\
\hline TDPP-QAS & $\mathrm{H}_{2} \mathrm{O}$ & $530 / 595$ & 47 & & & & [58] \\
\hline SeDPP-QAS & $\mathrm{H}_{2} \mathrm{O}$ & $537 / 618$ & 42 & & & & [58] \\
\hline Phenyl DPP 5 & PBS & $455 / 515$ & 57 & 22,700 & & 6.5 & [59] \\
\hline Thienyl DPP 6 & PBS & $530 / 596$ & 49 & 30,700 & & 4.4 & [59] \\
\hline Optimized & PBS & $456 / 548$ & 76 & 14,700 & \multirow{4}{*}{1350} & 6.5 & \multirow{2}{*}{ [59] } \\
\hline phenyl DPP 7 & $\mathrm{H}_{2} \mathrm{O}$ & $456 / 548$ & 94 & 14,700 & & 6.8 & \\
\hline Optimized & PBS & $527 / 594$ & 56 & 18,100 & & 4.8 & \multirow{2}{*}{ [59] } \\
\hline thienyl DPP 8 & $\mathrm{H}_{2} \mathrm{O}$ & $527 / 594$ & 80 & 18,100 & & 5.0 & \\
\hline \multirow{2}{*}{ DPP 1} & DCM & $609 / 636$ & 48 & 43 & 27 & & \multirow{2}{*}{ [42] } \\
\hline & PBS & $576 / 635$ & 2.0 & 42 & 59 & & \\
\hline \multirow{2}{*}{ DPP 2} & DCM & $608 / 635$ & 42 & 48 & 27 & & \multirow{2}{*}{ [42] } \\
\hline & PBS & $575 / 637$ & 1.0 & 47 & 62 & & \\
\hline \multirow{2}{*}{ PhDPP } & $\mathrm{MeCN}$ & $471 / 556$ & 20 & & 3246 & 6.21 & \multirow{2}{*}{ [15] } \\
\hline & $\mathrm{H}_{2} \mathrm{O}$ & $481 / 604$ & 8 & & 4234 & 2.33 & \\
\hline \multirow{2}{*}{ PhODPP } & DMSO, & $475 / 550$ & 95 & & 2871 & 6.38 & \multirow{2}{*}{ [15] } \\
\hline & $\mathrm{H}_{2} \mathrm{O}$ & $481 / 599$ & 56 & & 4096 & 3.62 & \\
\hline M1 (OMe) & $\mathrm{THF}$ & $501 / 646$ & 1.1 & & & 0.32 & [60] \\
\hline M2 (OMe) & $\mathrm{THF}$ & $514 / 625$ & 1.3 & & & 0.53 & [60] \\
\hline D1 no OMe & $\mathrm{THF}$ & $495 / 611$ & 46.2 & & & 2.89 & [60] \\
\hline D2 no OMe & THF & $506 / 595$ & 67.5 & & & 3.10 & [60] \\
\hline T2 (TPA) & THF & $503 / 590$ & 71 & 30,000 & 87 & & [61] \\
\hline D2 (TPA) & THF & $504 / 602$ & 68 & 42,200 & 98 & & [61] \\
\hline M2 (TPA) & $\mathrm{THF}$ & $513 / 625$ & 2 & 50,500 & 112 & & [61] \\
\hline
\end{tabular}

FPs, fluorescent probes; FL, fluorescence lifetime.

Series of DPP-based FPs coded (7a-f), bearing peripheral substituents of $(\mathrm{H}, \mathrm{Me}, \mathrm{CN}$, $\mathrm{SMe}, \mathrm{OMe}$ and $\mathrm{NMe}_{2}$ ), were synthesized, purposely, to study their photophysical effects both for electron-donating and withdrawing substituents to the central DPP for different chromophores independently (Figure 3) ${ }^{56}$ [56], as very little is known from the literature for this strategic approach. Only derivative 7e having strong electron-donating $\mathrm{NMe}_{2}$ was strongly quenched by displaying FQY of 3\% with absorption and emission peaks nearing the infrared region at 684 and $753 \mathrm{~nm}$, respectively, tested in DCM solution; however, this nonstructural emissive band exhibited the biggest Stoke's shift of $1322 \mathrm{~cm}^{-1}$ among them. The bathochromically shifted absorption band along with observed FQ of 7e results from the planarity via extended $\pi-\pi$ conjugation system of the bithiophene motifs towards the acceptor unit (the central DPP). Consequently, FQY for 7a $(\mathrm{H})$ was $36 \%$, whereas both $7 \mathrm{~b}$ and $7 f$ with Me and CN, respectively, show 32\%, and were also red-shifted to NIR with absorption and emission bands range of 613 to $656 \mathrm{~nm}$. 

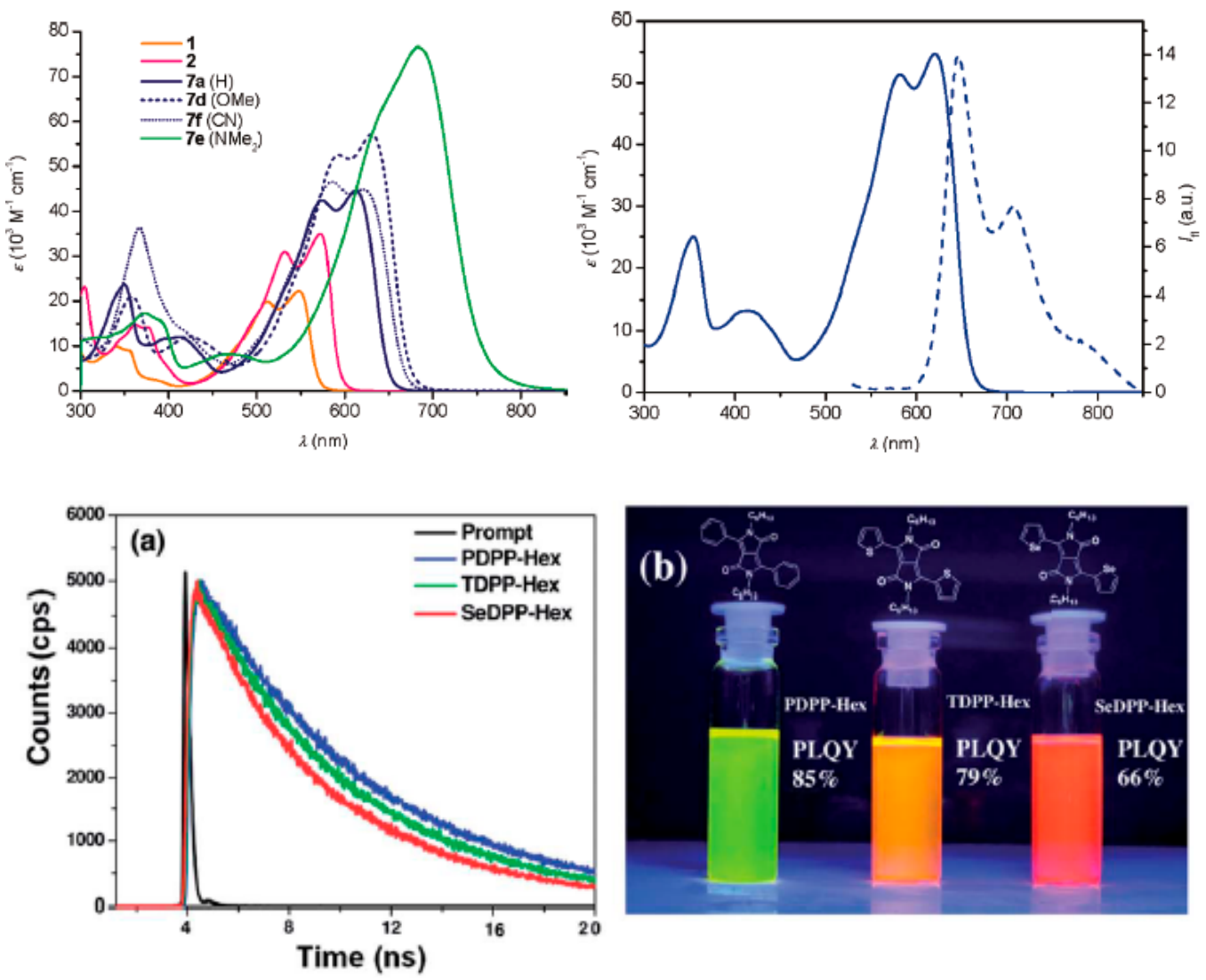

Figure 3. Row-1: left, the DPP derivatives 1, 2 and 7a,d-f UV-vis spectral absorption in DCM $\left(2 \times 10^{-5} \mathrm{M}\right)$ at $20^{\circ} \mathrm{C} .7 \mathrm{~b}$ (Me) and 7c (SMe) shows very clear comparable spectral features with 7d (OMe); Right-hand side, spectral features in DCM Absorption (solid line) with emission (dashed line, excited at $520 \mathrm{~nm}$ ) of DPP $7 \mathrm{~b}(\mathrm{Me})$ at $20^{\circ} \mathrm{C}$. Copyright, 2011, reproduced with permission from Reference [56]. Row-2: (a) fluorescence decay pattern of varied DPPs having dissimilar donor units. (b) Pictures showing varied radiating behaviors of three differently prepared DPPs illuminated under ( 365 nm) UV light. Copyright, 2014, reproduced with permission from Reference [57].

Later on, Dhar et al. reported an observed decreased in FQY of $85 \%, 79 \%$ and $66 \%$ for phenyl, thiophene and selenophene DPPs, showing green, orange and red colors (different wavelengths), respectively (Figure $3 a, b)^{57}$; such declines were ascribed to the effective heavier nature of sulfur and selenium atoms which favors inter-system crossing (ISC), and similarly their fluorescence lifetime (FL) decreased in the same way with decayed lifetime from 6.8 , to 6.2 and $5.5 \mathrm{~ns}$, respectively, for phenyl, thiophene and selenium derivatives [57]. Their absorption spectra show dual emission bands at 510 and $525 \mathrm{~nm}$ for thiophene derivative and then 550 and $562 \mathrm{~nm}$ for SeDPP-Hex, but PDPP-Hex displayed lowest energy band at $470 \mathrm{~nm}$. Their fluorescence spectra displayed a bathochromic redshifted emissive bands of 535, 565 and $580 \mathrm{~nm}$ for phenyl, thiophene and selenophene, correspondingly, with displayed dual-band vibronic emission. Another quenched in FQYs was also reported by Wang et al. with DPPs bearing quaternary ammonium salts (QAS) used for investigating their levelling efficiency in copper electroplating technology. The coded FPs are; FDPP-QAS (55\%) a furan-based, TDPP-QAS (47\%) a thiophene-based and for selenophene-based is SeDPP-QAS (42\%) shows a correspondingly similar gradual decline in their observed FQY which looks bright for these dyes after illuminated with UV light at $365 \mathrm{~nm}$ [58].

In another development later in 2015, authors of Reference [59] demonstrated an intensely slight bathochromic shift at 548 and $553 \mathrm{~nm}$, respectively, with FQYs of 57\% and $49 \%$ for a phenyl and thienyl DPP derivatives coded 5 and 6 having carboxylic acid at their terminal ends. These materials happen to reveal an intense absorption at 460 $\mathrm{nm}$ with emission at $530 \mathrm{~nm}$ in PBS solution of $\mathrm{pH}=7.4$ for both DPP-phenyl and DPP- 
thienyl respectively. Further modification of these dyes with a taurine-like disulfonated substituent, phenyl and thienyl derivatives coded 7 and 8 are obtained Table 2 above, and such optimization boosted their FQYs to $76 \%$ and $56 \%$ respectively. Upon binding these dyes with BSA protein, the spectral pattern of bound-dyes (BSA-7 and BSA-8), compared to unbound-dyes, remains the same with very high FQYs implying a fluorescence turnon event, which are slightly lower by only $20 \%$ in relation to the freely unbound-dyes. However, the DPP-phenyl exhibited a bigger Stoke's shift of $1350 \mathrm{~cm}^{-1}$ with an evident vibronic band sequence vividly observed in contrast to the DPP-thienyl. Their FL was found to be (4.5 to $6.5 \mathrm{ns)} \mathrm{similar}$ with most commonly soluble ODs. Tang et al. (2017) presents other FPs coded DPP1 and DPP2 incorporated with heterocyclic aryl amine flanked to the DPP, with each bearing another free carboxylic acid moiety for conjugation with biomolecules. Absorption spectrum of these dyes shows a vibronic with shoulder peaks around 565 and $578 \mathrm{~nm}$ in DCM, DMSO and MeCN, nonetheless, their electronic absorption in PBS were slightly blue-shifted to $575 \mathrm{~nm}$ and conversely, their emission spectra lies mainly in far-red region. These dyes experience a decreased FQYs in different organic solvents, were 0.48 and 0.42 (DCM), 0.38 and 0.26 (DMSO), and 0.13 and 0.16 $(\mathrm{MeCN})$, displayed for DPP1 and DPP2, respectively. Solvent polarity effect leads to the observed FQY decline from DCM to DMSO through (MeCN) [42]. Such decline in FQY was found even more pronounced in PBS pH 7.4 with 0.01 for DPP1 and 0.02 for DPP2 dyes, probably this is favored due to the hydrogen-bonding, as such leads to the generation of an electron-accepting water cages which might be possible for heterocyclic amines. However, upon conjugation with BSA protein, both dyes displayed a strongly enhanced fluorescence emission especially with DPP2 an 18-fold FQY increased a protein-induced fluorescence enhancement (PIFE), implying protein hydrophobicity to have greatly restricted boundBSA of DPPs to vibrate. These findings contradict BSA-7 and BSA-8 bio-conjugation previously discussed here, in this section [59].

\subsection{Photophysical Effects of Triphenylamine (TPA) on DPP Fluorophores}

It has been established, by Cai et al., that incorporating DPP with triphenylamine (TPA) significantly weakens the emissive properties of the resulting material DPP-TPA [62], this originate after investigating the DPP-TPA PLQY to be 0.09 whereas the individual photoluminescence quantum yield (PLQY) of DPP and TPA are 1 and 0.03 respectively this coincides with time-resolved photoluminescence (TRPL) investigation. Accordingly, their FL significantly declines after conjugating to form DPP-TPA as DPPs FL was found to have decreased from 6.8 to $2.6 \mathrm{~ns}$ and for the TPAs from 2.2 to $1.2 \mathrm{~ns}$. These data propose the establishment of an extra non-emissive decay canals which likely opened upon conjugation. Hence, this implied that individual fragments of these molecules are consumed inter-connectedly via phonon coupling and therefore produce heat. As a result, it could be inferred that DPP-TPA transformation from light to heat initiates as a result of the boosted light absorption with correspondingly decreased in emitted light after conjugation. Following this findings, Hwang et al. reported a substantial FQ of a DPP-based D-A $\pi$-conjugated material having Dimethoxytriphenylamine moieties (Row-1, Figure 4 , graph $)^{60}$, which resulted from the significantly induced intramolecular photoinduced electron transfer (PET) of the methoxy (OMe) moiety on the molecular frame of this highly emissive and prominent DPP fluorophore [60]. This obviously known intramolecular PET results greatly in quenching because it is a photo-deactivation process; thus, it affects the photoluminescence (PL) of this electronically donor-acceptor conjugated materials having TPA composed of OMe moieties (M1, M2) where the PET originates. However, other derivatives with TPA having no OMe moieties (D1, D2) fluoresce strongly. This is the first time pronounced FQ from the OMe moiety via intramolecular PET for donor/acceptor conjugated molecules was reported. The quenching phenomena was detected in solution, aggregates and even solid-state as a result of PET. This is because HOMO energy levels of OMe-containing DPP contributed largely to the non-bonding $n$ states, and hence, this was found to be higher for DPP bearing zero OMe TPA and so 
contributed largely for its binding states. Hence, the driving forces exert more energy for M1 and M2 in relation to D1 and D2 with no OMe moieties. Synthesized DPPs having TPA groups existed almost non-luminous in purely NMP solvent with $0 \%$ water, though, they exhibited an increased luminous behavior of 17.4, 33, 8.3 and 6.1 FQYs at $90 \%$ water concentration respectively (Row-1, Figure $4 a-d$ ) ${ }^{60}$. This displayed phenomena was considered to be AIE solely due to the rotatable unrestricted TPA moiety. It is, therefore, clear that these synthesized DPPs aggregated in solution with high water concentration. Hence, the non-radiative decay was inverted to be radiatively emissive through restriction of intramolecular rotation (RIR) of rotatably free TPA motif. Interestingly, optical properties of these derivatives were studied by fabricating thin-films of D1, D2, M1 and M2, and their emission wavelengths are $616,611,648$ and $640 \mathrm{~nm}$, respectively, with displayed FQYs of 24.8, 24.2, 12.6 and 8.6\%, correspondingly. In solid-state in spite of the various photo-deactivation pathways like the intermolecular stacking and aggregates formation, likely the AIE properties of these prepared materials with TPA motifs acted significantly in sustaining the thin-films solid-state emanation.

In the same year 2019, another series of DPP FPs bearing three different TPA functional groups T2, D2 and M2 were prepared by the same authors [61], which were all studied in solution of water/THF mixture and solid films. These materials were all red-shifted along with AIE properties (Row-2, Figure $4 a-d$ ) ${ }^{61}$, but with varied glowing properties in the red-shifted region which was due to the variation of the TPA moieties attached to each derivative. Their FQY in solid-state on thin-films gave $31 \%, 26 \%$ and $9 \%$, respectively, at emission wavelengths of 614, 611 and $640 \mathrm{~nm}$ for T2, D2 and M2. Their absorbance and PL spectra all displayed a bathochromic red-shift by certain factors, when compared to their spectra tested in pure THF solution. However, the observed low FQY in their solid films, when compared with that in pure THF (71, 68 and 2 for T2, D2 and M2) from Table 2, is attributed to the aggregation-caused quenching (ACQ), which originates from the planarity of the organic fluorophore aggregates, as a result of $\pi-\pi$ stacking and the intermolecular interactions. As can be seen in Row-3 of Figure $4 a-d^{61}$, surface roughness of T2 and D2 is greater, in addition to their more $\pi-\pi$ stacks assembling with the intermolecular interactions. In a nutshell, their AIE develops from RIR process and TPA motifs, which influence the ACQ in a trade-off relationship. Thus, T2 and D2 have AIE properties in their solid thin-film with a very high FQY than M2. 

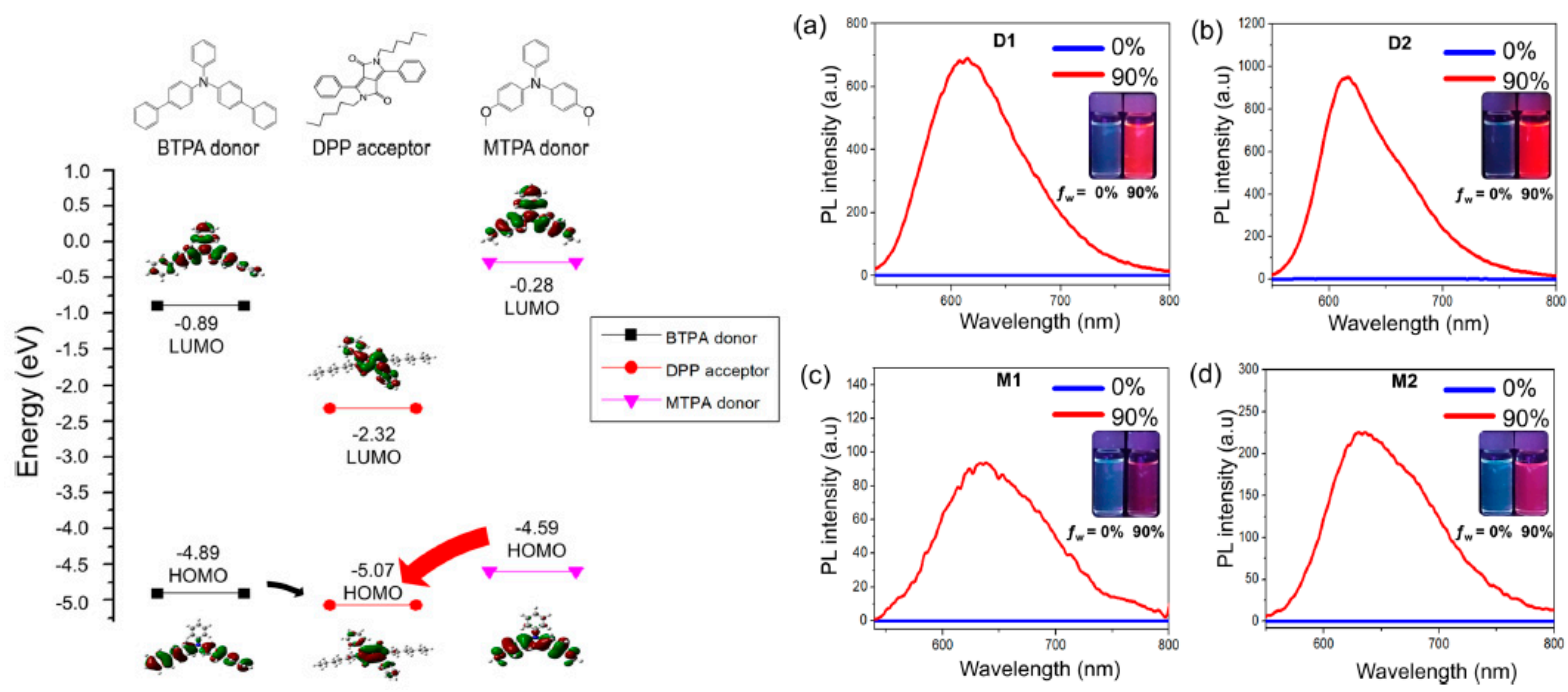

(a)
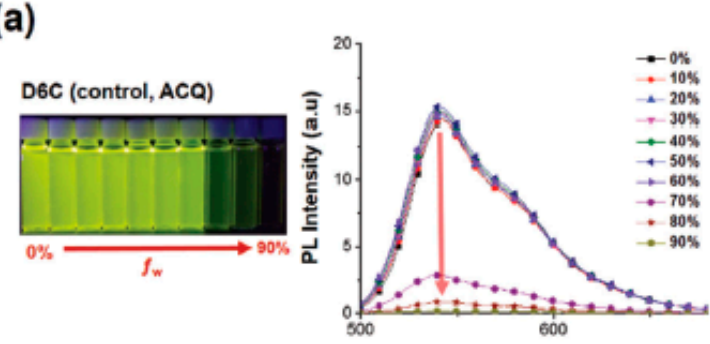

(c)

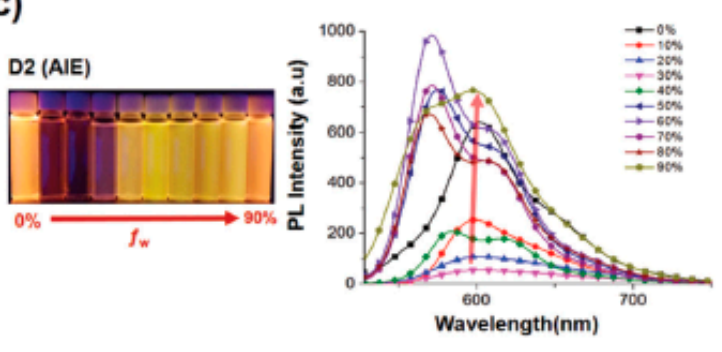

(b)

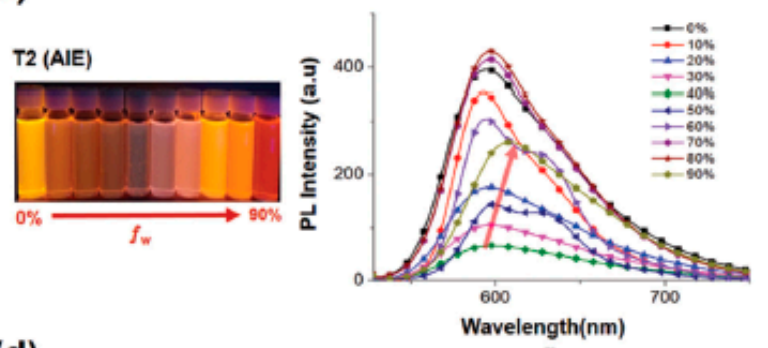

(d)

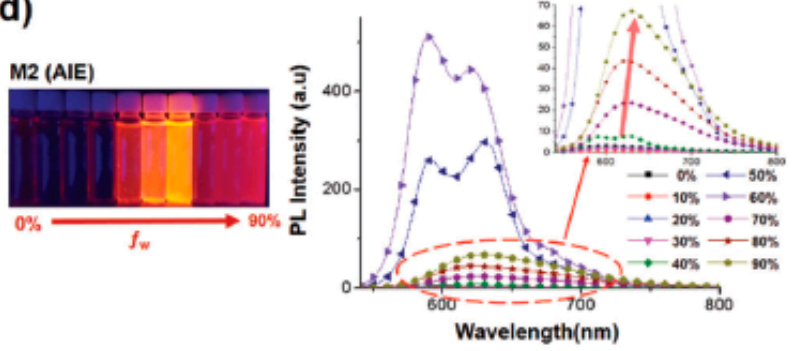

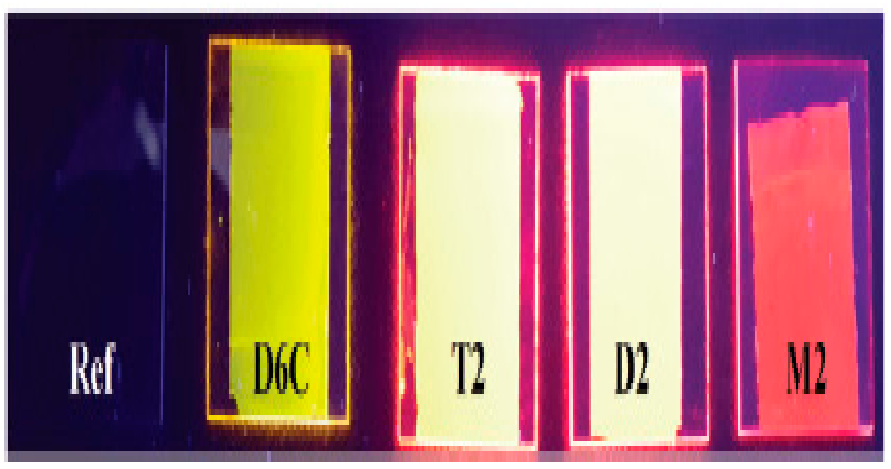
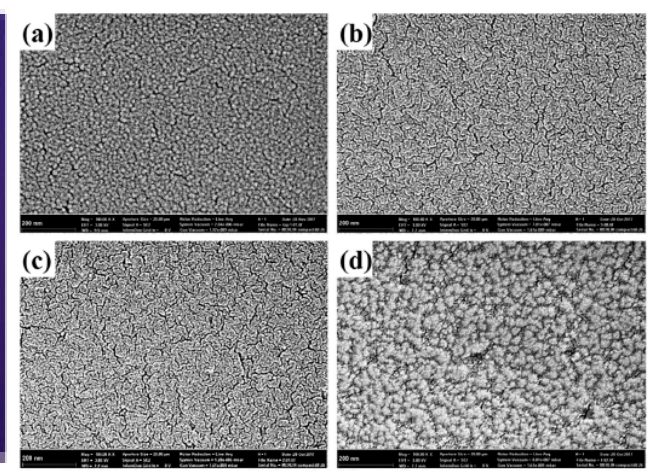

Figure 4. Row-1: left, designed energy levels of donor to acceptor units. Right, PL intensity of synthesized DPPs with their resulting optical images in NMP $/ \mathrm{H}_{2} \mathrm{O}$ mixtures, using varied water contents from $\left(0 \%: 10^{-5} \mathrm{~mol} / \mathrm{L}\right.$ in NMP. $90 \%$ : $10^{-5} \mathrm{~mol} / \mathrm{L}$ in NMP $/ \mathrm{H}_{2} \mathrm{O}=1: 9(v / v)$. Copyright, 2019, reproduced with permission from Reference [60]. Row-2: (a) D6C, (b) T2, (c) D2 and (d) M2 are the visual images with PL spectra in THF/water mixtures at varied water contents $\left(f_{\mathrm{w}}\right)$. Copyright, 2019 reproduced with permission from Reference [61]. Row-3: left, visual images of D6C/PMMA, T2/PMMA, D2/PMMA and then M2/PMMA films. Right, Surface morphologies of DPP compound/PMMA in thin films by FE-SEM: (a) D6C, (b) T2, (c) D2 and (d) M2. Copyright, 2019 reproduced with permission from Reference [61]. 


\subsection{Sensitizers and Annihilators}

In an effort to transforming singlet fusion (SF) to triplet fusion (TF) for realizing new class of annihilators with DPPs, Shen et al. (2018) discovered that structural modification of the DPP fluorochromes indicates that DPP-based small molecules should be incorporated with aromatic units not more than one/two on either side for efficient solar cell applications. This analysis was conducted by exploiting the furan, thiophene and thiazole aromatic substituents flanked to the DPP core, and it was found that all DPPs attached to one unit with either furan, thiophene or thiazole have exhibited efficient SF [63]. Moreover, further modification of the flanked aryl unit to the DPP fluorophore with either electron-donating or -withdrawing groups results in decreasing the energy band gap. Research findings revealed that attaching electron-donating groups to the flanked aromatic rings at $\alpha$ or $\beta$ positions, like Me, produces an endothermic SF DPP material, whereas an attached electron-withdrawing group like F, at the same positions, generates an exothermic SF DPP.

Recently Chakali et al. presented a covalently linked charged-separated state SF by a newly synthesized BT-DPP-TPA(7) asymmetric molecule, composed of triphenylamine (TPA) and benzothiophene (BT) as donors with the acceptor unit DPP having thiophene spacer [5]. The ultrafast relaxation dynamics of such triad molecule was discovered by solution phase via a femtosecond transient absorption spectroscopy. A significant FQ of the DPP in the triad molecule was discovered via steady-state and time-resolved emanation studies. The optimized triad DPP molecule, owing to its facile redox potential generated a highly exothermic system, influences the formation of a moderately quicker photo-induced charged separation (CS) with a relaxed charged recombination (CR) processes (existence of a long-lived charged separated state). Reduction of PET takes place after photoexcitation of DPP moiety in the triad molecule from TPA ground-state to DPP's excited singlet state which leads to the CS process. It is worth noting, that BT-DPP-TPA(7) partake for the first time to solution phase SF with concentration above $100 \mu \mathrm{m}$ from 100 to $200 \mathrm{ps}$ time scale. The CS rate was found to be between $10^{9}$ and $10^{8} \mathrm{~s}^{-1}$, which rises up on moving from non-polar to polar solvents. On the other hand, CR happens to be very slow in nanoseconds in all solvents. It is therefore anticipated to note that photoexcitation of this novel triad molecule BT-DPP-TPA(7) to produce a longed-lived CS state which at the same time undergoes $\mathrm{SF}$ and, hence, serves as an SF sensitizer.

Following efforts by Pun et al. to develop new class of annihilators with DPP (a tunable upconversion emission), from SF to TF-in other words, triplet-triplet annihilation-DPP shows promising potential to generate new annihilators. This was demonstrated in a report about optimization of the suspended aryl substituents flanked to the DPP fluorophore resulting in altering the DPP's singlet energy to TF. This discovery denotes a leap in understanding how triplet fusion upconversion (TFU) mechanistic pathways are influenced by the molecular structure of the DPP molecules via chemical interaction. It was well documented that $\sim 1.15 \mathrm{eV}$ is the DPP's triplet excited-state energy; however, their singlet excited-state depends solely on the aryl groups flanked to the DPP chromophore since such substituents varied for diverse derivatives. As a result, 8 differently DPP-based FPs bearing dissimilar aryl units were successfully synthesized in order to assess the influence of varied aryl groups in modulating their singlet-energy-state to TFU output light as seen in (left, Figure 5A,B) ${ }^{64}$ [64]. These materials show decreased steady-state optical energy gap as evidence from their observed regular red-shifted absorption onset which serves as proof for the optical energy gap shrinkage. DFT further confirms DPP triplet-energy-state to be $\sim 1.15 \mathrm{eV}$, implying TFU for 1 and 2 derivatives not feasible as their singlet energies are 2.40 and $2.34 \mathrm{eV}$ (above $2.3 \mathrm{eV}$ ); however, TFU was possible from 3 up to the 8 derivatives as their singlet energies are $2.3 \mathrm{eV}$ lower (this is as a result of these values being lesser than double the DPP's triplet energy). The upconversion quantum yields (UQY) event for these molecules could be endothermic or exothermic which relies solely upon the nature of the flanked aryl unit attached to the DPP chromophore. Their TFU were tested using prominent sensitizing agent of (PdPc that absorbs in the NIR) by undergoing intersystem crossing (ISC) to produce triplet with $\sim 1.3 \mathrm{eV}$ sufficiently enough for DPP energy transfer. The study 
showed that three to eight annihilators exhibited singlet energies of 2.21, 2.16, 2.08, 2.00, 1.95 and 1.90 with corresponding UQY of $3.2,0.91,0.50,0.55,1.8$ and 1.1, respectively (right, Figure $5 A, B)^{64}$. The strength of electron-donating property of the aryl substituent is of high significance as strong electron-donating aryls result in lowering the optical energy band gap, thus, implying a singlet energy reduction with correspondingly low-energy PLQY. DPP material 3 displayed the largest UQY value of $3.2 \%$, lower than $5.5 \%$ for commonly used annihilators of rubrene. However, DPP exhibited an appreciable stability in ambient condition, thus making DPPs tougher in relation to acene-derivatives as their oxygen content makes them more prone to oxidation. Therefore, these findings clearly outlined the DPPs' extreme stability compared to acenes and can be utilized in developing newly innovative annihilators for TFU.
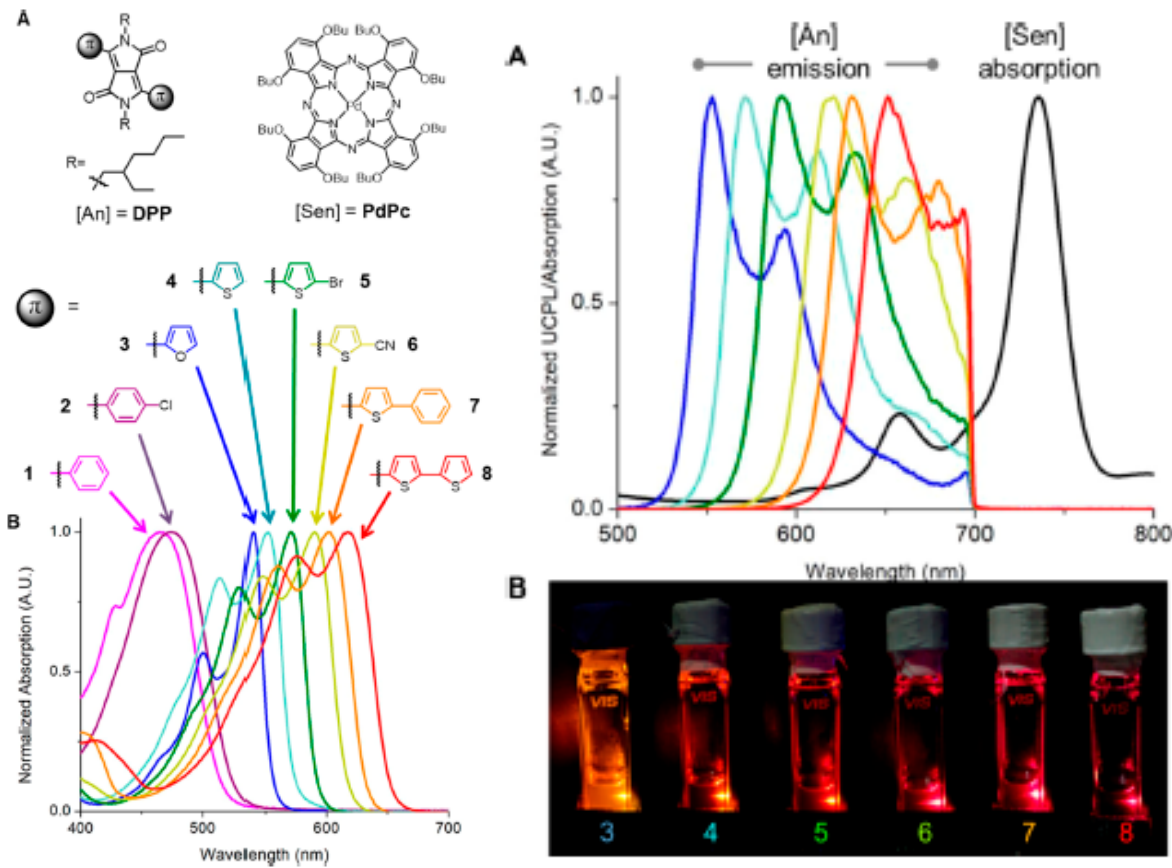

Figure 5. Left, (A) chemical structures of used DPP molecules in this work. (B) Normalized absorption spectra of the synthesized DPPs $(1-8)$ in toluene. Right, (A) normalized UCPL of molecules from 3 to 8 (blue, cyan, green, yellow, orange and red, separately) in toluene, excited by a $730 \mathrm{~nm}$ laser-diode, using a $700 \mathrm{~nm}$ short-pass filter. Black spectrum is the normalized absorption of PdPc (sensitizer) in toluene. (B) Is the UCPL pictures of small molecule DPPs 3-8. Copyright, 2019, reproduced with permission from Reference [64].

\section{Solid-State Fluorescence of DPPs with Their Aggregation-Induced Emission Enhancement (AIEE/AIE) in Solution and Water}

\subsection{Solid-State Emission}

Fluorescence in solid-state is of paramount importance in organic optoelectronics science, this is because organic fluorophores are an integral components of organic light emitting electronic devices like (OLEDs), solar cells [65], etc., as such applications needs such fluorophores to be fabricated into compacted solid films [66]. Therefore, the possibility of organic fluorophores to come together and aggregate to form solid films is the fundamental basis of aggregation-caused quenching (ACQ) phenomena, which significantly limits their biological applications like bio-imaging, as well as their organic electronics technological advancement.

\subsubsection{Aggregation-Induced Emission Enhancement (AIEE/AIE)}

In 2010, the authors of Reference [67] described four differently synthesized DPP FPs bearing distinct electron-donating groups attached to the phenyl-ring flanked to the DPP core via cross-coupling reaction (Graphical Figure $6{ }^{67}$, and these prepared DPPs are 
capable of displaying a red-shifted absorption and emission in solutions (Figure 6 spectra, left ${ }^{67}$. These materials show apparently high FQYs in solutions of $\mathrm{CHCl}_{3}$; however, only derivative bearing butoxyphenyl DPP was found emitting strongly in the solid-state, with an observed FQY of 0.45 . This is because the conjugation system in butoxyphenyl DPP is probably higher compared to its solution phase which is ascribed to the material's packing forces in the solid-state, hence preventing reabsorption of emitted photons due to high Stoke's shift that originated from the lengthy conjugated system which is likely the reason why its FQY been highly displayed in solid-state. Therefore, the stretchable butoxyphenyl DPP aggregated in solid-state possibly because of its lengthy conjugated network to DPP fluorophore coupled with the biphenyl functionality. Therefore, such attached substituents to the phenyl-ring affects the chemical properties of these DPP chromophores both in solution environment and solid-state greatly.

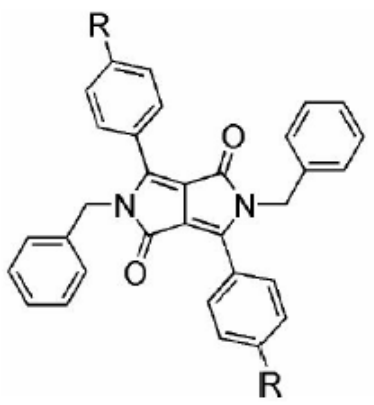

$1: \mathrm{R}=-\xi-\mathrm{Cl}$

$2: R=\xi-N$

$3: \mathrm{R}=$.ริ $\approx \mathrm{C}_{6} \mathrm{H}_{13}$

$4: \mathrm{R}=-\xi-\mathrm{OC}_{4} \mathrm{H}_{9}$
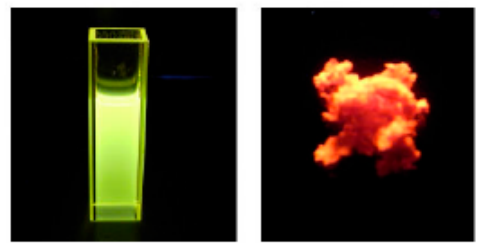

(a)

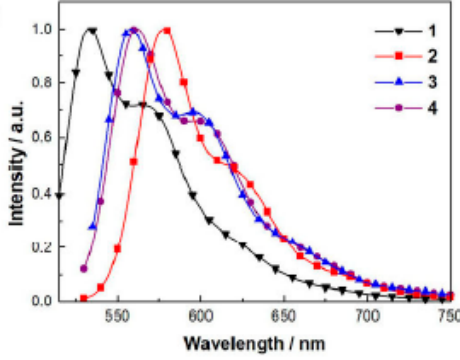

(b)
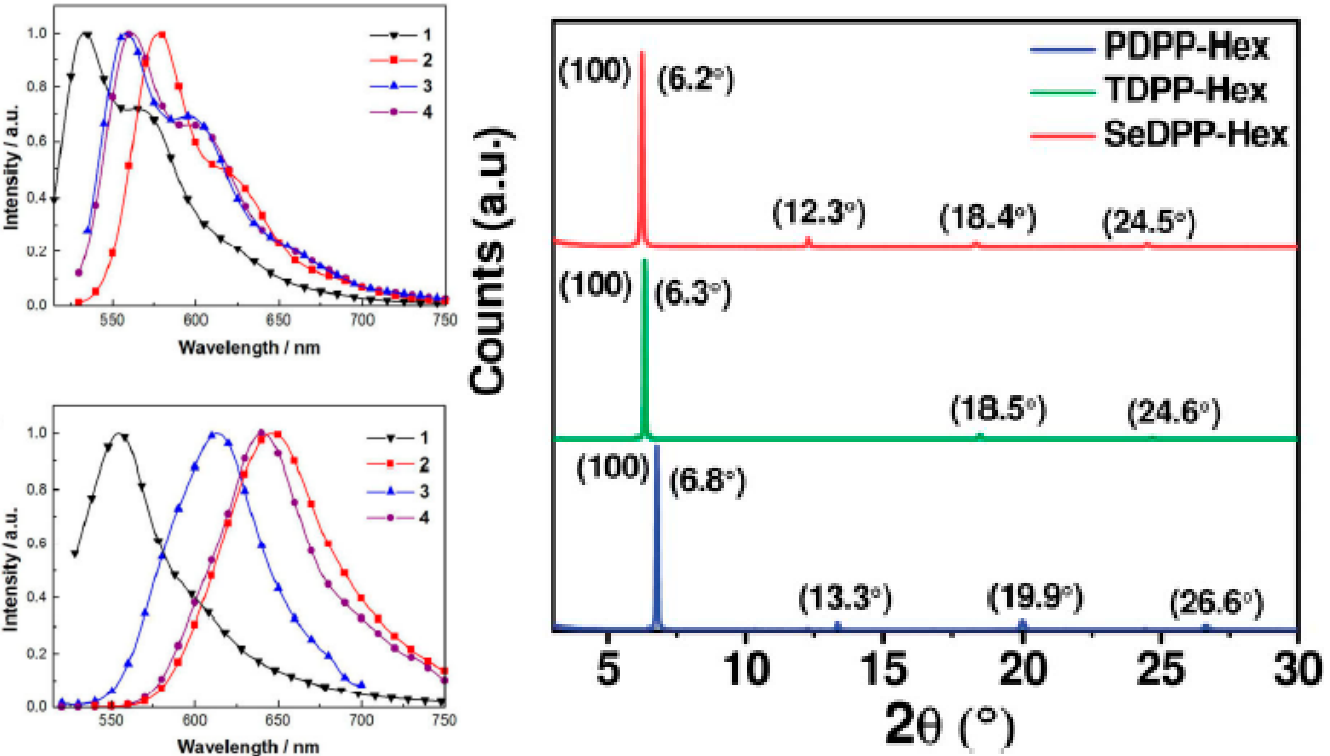

Figure 6. Row-1: Molecule structures of prepared fluorescent DPPs 1-4. Row-2: Left, their spectral emission (a) in $\mathrm{CHCl}_{3}$ and (b) in solid-state. Copyright, 2010, reproduced with permission from Reference [67]. Right, thin-film X-ray diffractogram deposited on silicon wafers of the three prepared DPPs. Copyright, 2014, reproduced with permission from Reference [57].

In order to get more light for AIE DPP FPs, a chain of three different derivatives with various substituent groups at 2,5 and 3,6 positions of the flanked DPP core (Scheme $2 \mathrm{~A}$ ) ${ }^{51}$ were prepared a year later 2011 by authors of Reference [51], in that the molecules $\pi$-conjugation along with their alkyl-chains shows great influence on the central DPP fluorophore unit during emission in solution and solid-state. Only 2,5-Dialkyl-3,6-bis(4-((10-oxoanthracen-9(10H)ylidene)methyl) phenyl)pyrrolo[3,4-c]pyrrole-1,4-dione series derivatives exhibited AIEE and crystallization-induced emission enhancement (CIEE). It was found that, 4a-c materials are all AIEE active both at $70 \% \mathrm{H}_{2} \mathrm{O} / \mathrm{THF}$ solution mixture which are 55 -fold higher compared to pure THF solution. However, the AIEE decreased from 56 to 5 with increased water content from $70 \%$ to $90 \%$, which was due to the morphological changes of the aggregate states in 
aqueous media. Below $70 \%$ water content fluorophore molecules slowly aggregates, thus forming more crystals with less amorphous aggregates but above $80 \%$ water content more amorphous aggregates are formed with lower proportion of crystals. In solid-state AIEE is much stronger than in solution which originates from their particle size and degree of crystallization that depends solely on the 2,5 substituents. Thin-film emission spectra of $4 \mathrm{a}$ material revealed to be CIEE active, this was observed through fumigation-induced emission (FIE) where 4a thin-film was fumed with $\mathrm{CH}_{2} \mathrm{Cl}_{2}$ vapor; afterwards, structural morphologies of non-fumed and fumed films were investigated, using polarization light microscope (POM), and it was confirmed that the enhancement in emission develops from the FIE, as untreated film displayed a uniformly dark shade under POM (Row-1, Figure 7A,B) ${ }^{51}$.

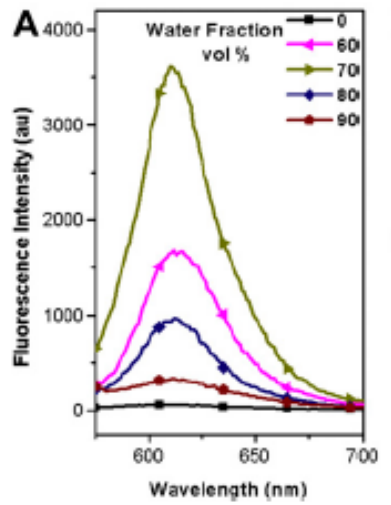

(a)

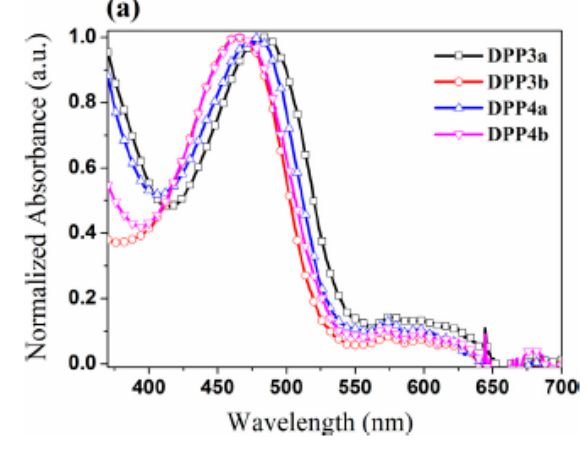

(a)

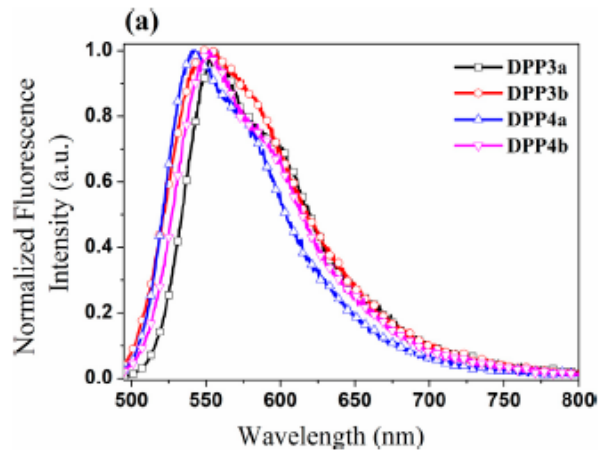

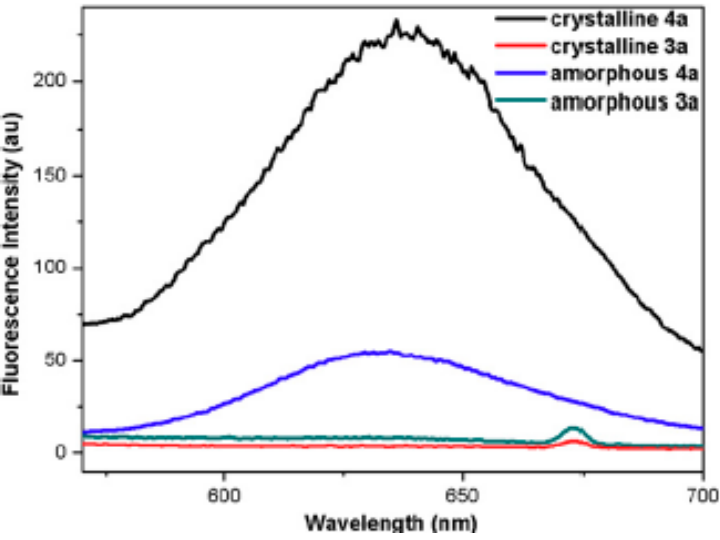

(b)

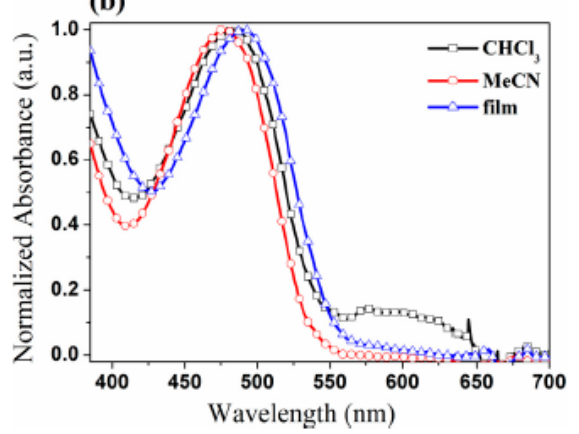

(b)

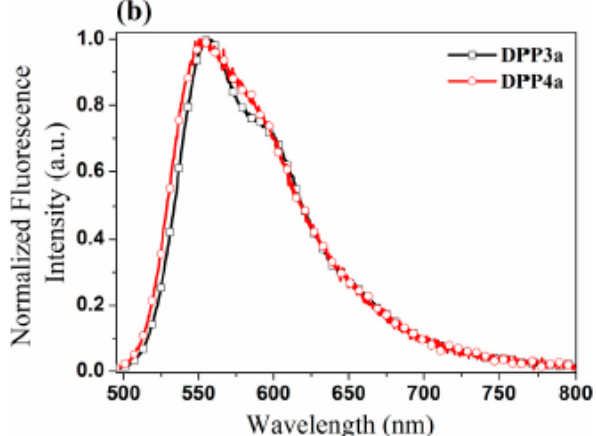

Figure 7. Row-1: left, (A) 4a spectral emission in $\mathrm{H}_{2} \mathrm{O} / \mathrm{THF}$ mixture. (B) Graphical representation of $\mathrm{I} / \mathrm{I}_{0}$ against water fraction $\left(f_{w}\right)$ in $\mathrm{H}_{2} \mathrm{O}$ /THF mixture. $\mathrm{I}_{0}$ and I are the 4 highest fluorescence intensity in THF and $\mathrm{H}_{2} \mathrm{O} / \mathrm{THF}$ mixtures $(10 \mathrm{mM})$, correspondingly; excited at (560 nm 4a), (520 nm 4b), and for 4c is $552 \mathrm{~nm}$. Right, spectral emission for amorphous $3 a\left(\lambda_{\text {ex }}=450 \mathrm{~nm}\right)$ while $4 \mathrm{a}$ $\left(\lambda_{\mathrm{ex}}=560 \mathrm{~nm}\right)$ is for crystalline films chromophores. Copyright, 2011, reproduced with permission from Reference [51]. Row-2: (a) evaluation of the normalized DPP3 $(\mathbf{a}, \mathbf{b})$ and DPP4 $(\mathbf{a}, \mathbf{b})$ dyes absorption spectra in $\mathrm{CHCl}_{3}$. (b) Evaluation of the normalized DPP3a dye absorption spectra in varied solvents and, on thin-film. Row-3: (a) evaluation of the normalized fluorescence spectral emission of prepared DPP dyes in $\mathrm{CHCl}_{3}$ (b) Evaluation of the normalized fluorescence spectral emission for DPP3a and DPP4a dyes on thin-films $\left(\lambda_{\mathrm{exc}}=485 \mathrm{~nm}\right.$ ). Copyright, 2019, reproduced with permission from Reference [68]. 
Following such development, in 2019, another four novel DPP-based FPs coded as DPP3a, DPP3b, DPP4a and DPP4b were prepared and experimentally studied both in solutions of (chloroform, acetonitrile) and solid-state (thin-films) [68]. All the dyes are somewhat blue-shifted and displayed a very low-lying broad band about $600 \mathrm{~nm}$ in chloroform solution (Row-2 images) ${ }^{68}$, this striking feature is attributed to the ICT that arises between the DPP core and indole ring unit. However, the observed red-shift at $600 \mathrm{~nm}$ in more planar $\mathrm{MeCN}$ solution was not identified. Such interpretations are consistent in consideration to the poorer polarizability of the dyes excited states with regards to their ground state in the tested solvent, and this consequently raises the LUMO of these dyes which hindered charge transfer from indole ring to the central DPP core unit. Absorption spectra of DPP3a and DPP4a are red-shifted in studied solutions compared to DPP3b and DPP4b, this is due to the induced resonance by the attached ethylhexyl to the ring systems of DPP3a and DPP4a by donating their electrons to the nitrogen atom of the DPP via sigma bonds. Similarly, these derivatives move slightly red-shift when testing their solid thin-films (to about $4-19 \mathrm{~nm}$ ) in relation to their absorption spectra in solution, thus implying the existence of $\pi-\pi^{*}$ interaction which resulted from the occurred molecular packing in their solid-state.

Accordingly, emission spectra for all the synthesized dyes were also studied, and the DPP core displayed only two major emissive bands from 514 to $598 \mathrm{~nm}$ after excitation at $485 \mathrm{~nm}$ in solutions (Row-3, Figure $7 \mathrm{a}, \mathrm{b})^{68}$; however, their emission maxima changes according to the polarity of the used solvent along with the attached side-chain nature. Only DPP3a moves slightly red-shift among these dyes in solutions of chloroform and $\mathrm{MeCN}$. Correspondingly, the emission spectra of DPP3a and DPP4a were tested on coated thin-films and displayed similar emission profiles. Moreover, even more interesting, is the fact that they move more easily and largely red-shift compared to their emission spectra in solution phases. This shows that DPP3a and DPP4a forms aggregate in solid-state with pronounced fluorescence property, suggesting its potential as AIE.

Presently, Tao et al. reported three DPP small molecule FPs based on furan possessing an unsymmetrical fluorochromes structures of D-A-D- $\pi$ for ICT characteristics [69]. The molecules are coded F1, F2 and F3, in that these dyes absorption and emission spectra displays an ACQ event in solution of THF/ $\mathrm{H}_{2} \mathrm{O}$ mixture. It is anticipating to note that dyes F1 and F3 displayed an AIE property in solution of DMSO/toluene mixture, whereas F2 DPP dye optimized with pyrene still shows an ACQ character. At the beginning, fluorescence intensity of F1 decreases and later increases stepwise; however, for F3, the intensity increases with increased toluene content in the DMSO/toluene solution mixture. The reason behind this possibly is due to the presence of two clear hydrogen-bond interactions which exist between the diketone oxygen atom with the $\beta$-hydrogen atom of the furan unit, which leads to enhanced molecular rigidity, and that were damaged in the THF $/ \mathrm{H}_{2} \mathrm{O}$ mixture. Possibly the observed AIE is likely ascribed to the RIR by the hydrogen-bonding. Furthermore, the energy band gaps 2.30, 2.29 and $2.35 \mathrm{eV}$ for F1, F2 and F3 correspondingly, gave us an insight that the incorporation of unsymmetrical furan-based containing DPPs to significantly reduce the HOMO-LUMO gaps.

\subsubsection{Molecular Packing Mode and Self-Assembling}

As a result of the promising nature of organic molecules self-assembly in enhancing their supramolecular architecture and morphology in solid-state for optoelectronics application [70], a DPP-based FP bearing an amphiphilic unit was designed and synthesized $\left(\mathrm{DPP}_{\mathrm{amphi}} \text { ) (top, Figure } 8 \mathrm{a}\right)^{71}$ by Mei et al. and has shown a little bandgap energy of $1.6 \mathrm{eV}$ [71], with good assembling in solution and bulk (top, Figure $8 \mathrm{~b})^{71}$. Absorption spectra of $\left(\mathrm{DPP}_{\mathrm{amphi}}\right)$ displayed broad absorption peak at $\sim 750 \mathrm{~nm}$ (bottom, Figure $\left.8 \mathrm{a}\right)^{71}$, with a corresponding change in color from blue to purple (DPP amphi solution in good solvent THF poured to poor solvent hexane), thus implying the presence of a robust exciton interactions with establishment of $\pi-\pi$ stacks. A peak at $680 \mathrm{~nm}$ illustrates the stoichiometric conversion of free molecules into aggregates. The DPP ${ }_{\text {amphi }}$ was found emissive in solutions of 
good solvent (THF); however, it was non-emissive in its thin-films (solid-state). This can be explained from the fact that the strongly $\pi-\pi$ stacking usually results in non-emitting crystalline materials due to prohibited little exciton shift. Thus, the solution study revealed the capability of DPP ${ }_{\text {amphi }}$ to self-assemble into extremely ordered nanostructures possibly via solvophobic effects and $\pi-\pi$ interactions synergistically.

a)

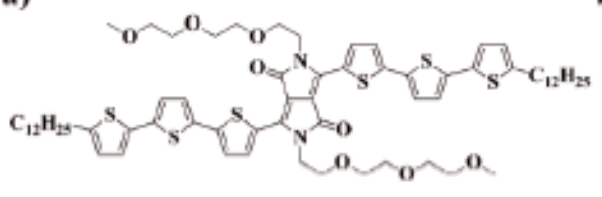

c)

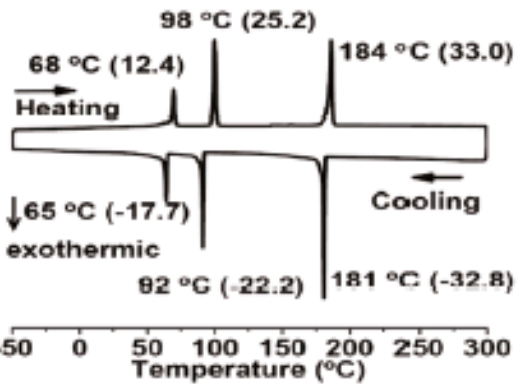

a)

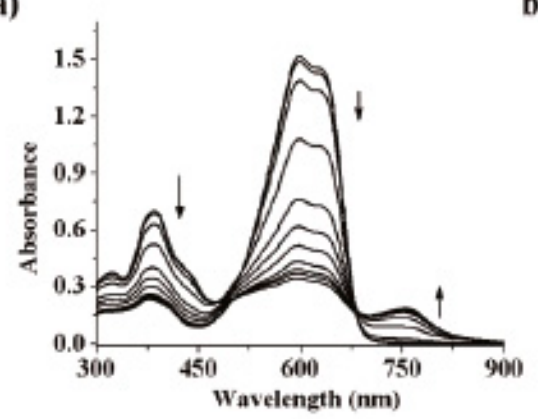

b)

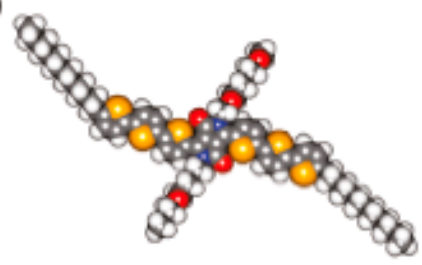

d)
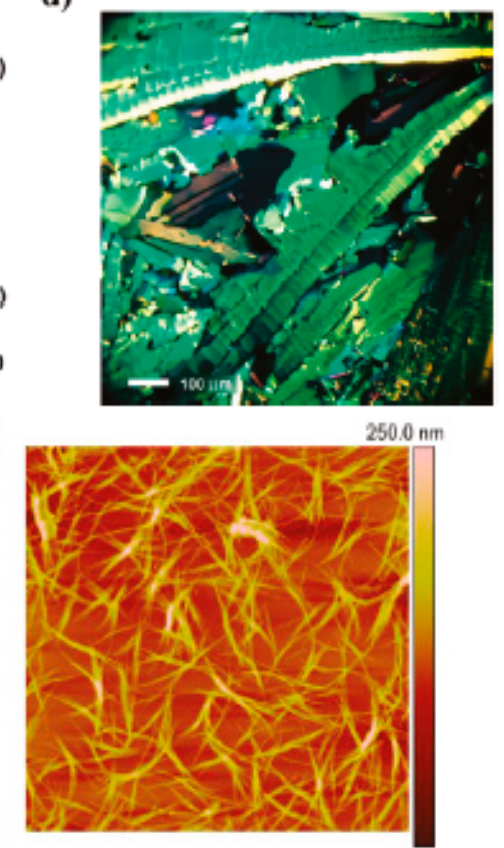

Figure 8. Top: (a) DPP ${ }_{\text {amphi, }}$ molecule structure, (b) DFT-optimized geometry space filling prototype (atomic colors), (c) DSC thermos-gram $\left(\mathrm{kJ} \mathrm{mol}^{-1}\right)$, and (d) DPP amphi polarized light microscopy at $170{ }^{\circ} \mathrm{C}$ (while on shearing). Bottom: (a) DPP amphi absorption spectra in methylcyclohexane $\left(2.2 \times 10^{-5} \mathrm{M}\right.$, at $\left.30^{\circ} \mathrm{C}\right)$ for $45 \mathrm{~s}$; (b) AFM morphology in tapping mode $(20 \times 20 \mu \mathrm{m})$ of DPP amphi put onto mica. Copyright, 2011 reproduced with permission from Reference [71].

In another study by Dhar et al., the effect of electron-donating phenyl, thiophene and selenophene group were studied for PDPP-Hex, TDPP-Hex and SeDPP-Hex derivatives correspondingly via single crystal $X$-ray diffraction technique and have shown highly ordered crystalline pattern (Figure 6, right) ${ }^{57}$ [57]. The study revealed a feebler intramolecular interaction for PDPP-Hex which results from the bulk repulsion of phenyl-ring ortho hydrogen with neighboring alkyl-chain hydrogen atoms and consequently leads to unfavorable torsional angle for PDPP-Hex. Conversely, thiophene and selenophene DPPs presents a slithered assembling connection with efficiently stabilized $\pi-\pi$ orbital overlap to the central DPP acceptor unit for S and Se donors. SeDPP-Hex exhibited powerful diffusion with great polarizability which was indicated from the significantly varied and altered C-X (X = S, Se bonds) for S ... O and Se ... O spaces. Moreover, this discrepancy greatly affects the optical band gap and molar absorptivity which increases from phenyl through thiophene to selenophene derivatives. As selenium is less electronegative (2.55) in relation to sulfur (2.58) with the additional lower aromaticity of selenophene compared to thiophene, this makes selenophene-ring to act as a strongly electron-donating moiety 
and this leads to significant stabilization of the excited-state of SeDPP-Hex. Likewise, the d-orbital delocalization is considered to be higher for selenium than in sulfur which resulted from the larger and sufficiently diffused d-orbital of selenium, this again indicates the observed maximum molar absorptivity of SeDPP-Hex derivative.

High device performance largely depends on the solid-state packing mode of materials precisely the well-defined molecular structures of small molecules and polymers. For this reason, Pang et al. tested three DPP-based organic small molecules of DPPCC, DPPBN-O and DPPBN-I (Scheme 1c) ${ }^{50}$ above, by exchanging C-C of terminally incorporated fluorine unit to the DPP acceptor with polar $\mathrm{B} \leftarrow \mathrm{N}$ moiety with varied orientation [50]. As $\mathrm{B} \leftarrow \mathrm{N}$ and $\mathrm{C}-\mathrm{C}$ bonds are isoelectronic to each other, this refined structural modification, changing the electronic features with further extra intermolecular dipole-dipole interactions, which results from the anionic and cationic characters of boron and nitrogen atoms, respectively. Optical absorption and fluorescence spectral changes of these molecules in solution and thin-films shows an intense broad absorption band from 470 to $700 \mathrm{~nm}$ which was recognized from the ICT, and such observed bathochromic red-shift by the solid thin-films was ascribed from the intermolecular interactions. Solid films thermal annealing of the inward and outward DPPs exhibited a predominant J-aggregation (head-tail $\pi-\pi$ stacking); however, DPPCC displayed H-aggregates (molecules stacked in a face-face orientation). In 2015, Ghosh et al. presented three differently designed DPP FPs offering electron-withdrawing nature in good yields (Figure 9, left) ${ }^{72}$. Thin-films absorption spectra of these derivatives showed a broad absorption between 600 and $700 \mathrm{~nm}$ with an onset at $750 \mathrm{~nm}$ (Figure 9, right $)^{72}$. These material thin-films were red-shifted by $\sim 40 \mathrm{~nm}$, compared to their spectra in solution; however, DPP-ES and DPP-AM were greatly red-shifted due to the presence of electron-withdrawing property of CN groups in relation to DPP-BZ [72].
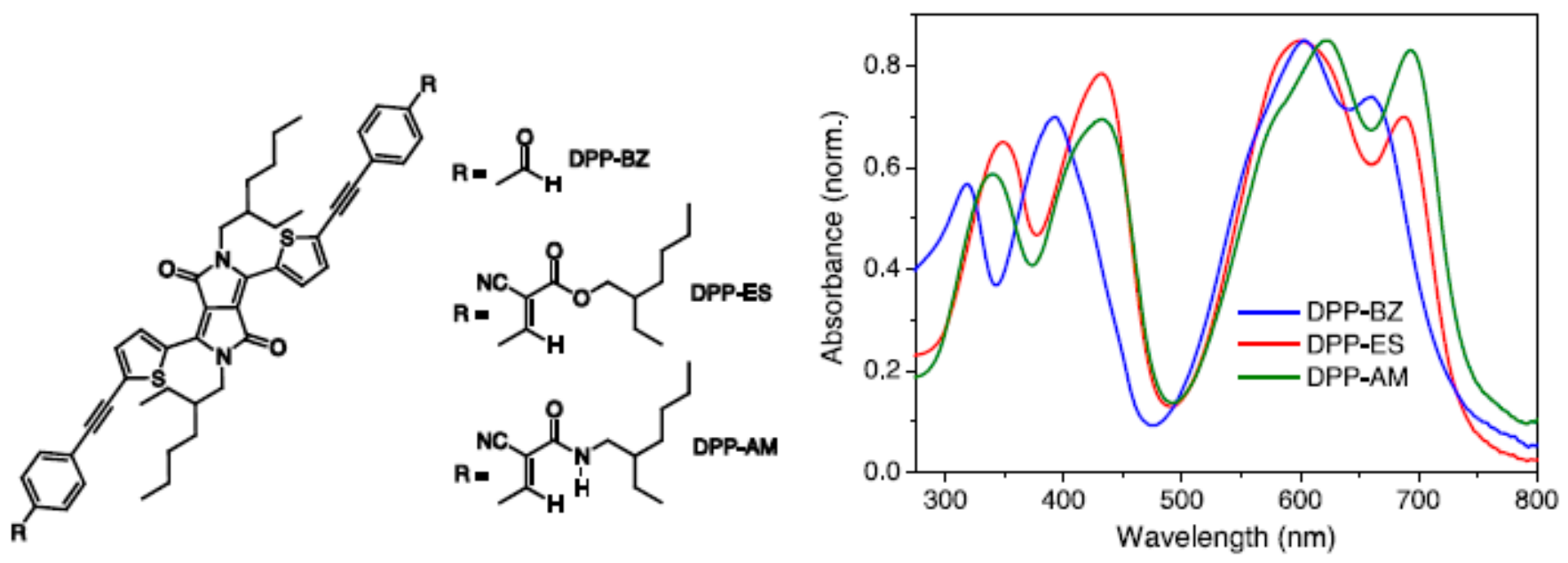

Figure 9. Left, synthesized DPPs Structure. Right, synthesized DPPs absorption spectra as thin-films. Copyright, 2015, reproduced with permission from Reference [72].

Recently Brimble et al. reported the biological application of self-assembled monowith a cross-linked thiophene-diketopyrrolopyrrole (a peptide-TDPP) systems by conjugating with octapeptide HEFISTAH [73], and investigated their photophysical features in different environment (solution, gel, solid films). These materials demonstrated fibrous morphological nanostructures by AFM, SEM and develops hydrogels at neutral $\mathrm{pH}$ in water $(4 \mathrm{wt} \%)$, but abortive to assemble at $\mathrm{pH} 2$ and 9 in (acidic and basic solutions). The formed hydrogels serve as strong proof of the peptide assembly, as they are made at such a minimum concentration. UV-Vis absorption spectra of these synthesized bio-organic materials named two and three in solution phase strongly conform to the materials spectra in solid thin-films by displaying relatively similar spectral changes (Figure 10a,b) ${ }^{73}$, hence implying that their chromophore structures are well-packed and maintained in solid-state 
(Figure 10c ${ }^{73}$. Therefore, it could be inferred that solution-phase probes for self-assembling can be utilized in projecting the solid-state features of such bio-organic components.

a)

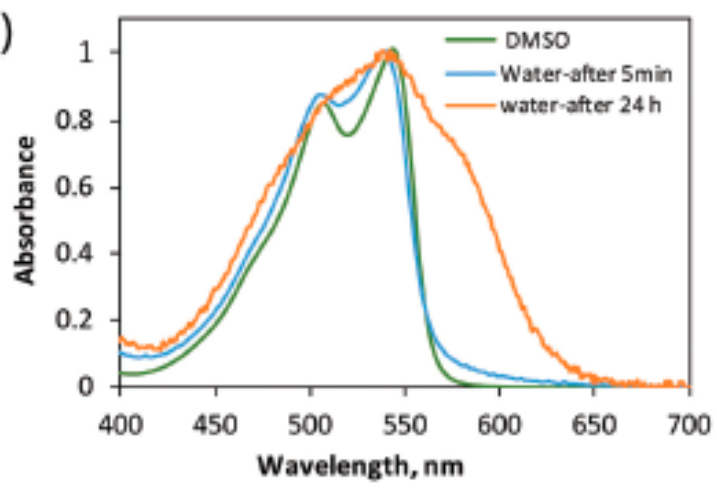

b)

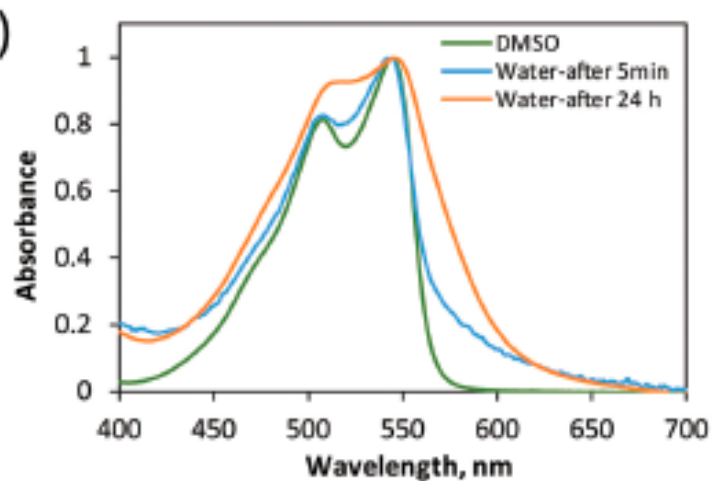

c)
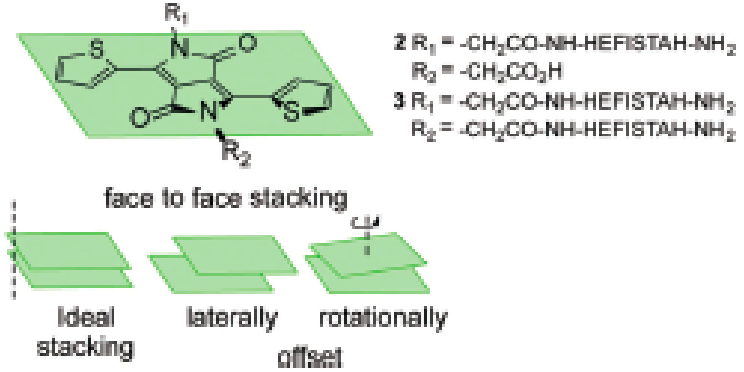

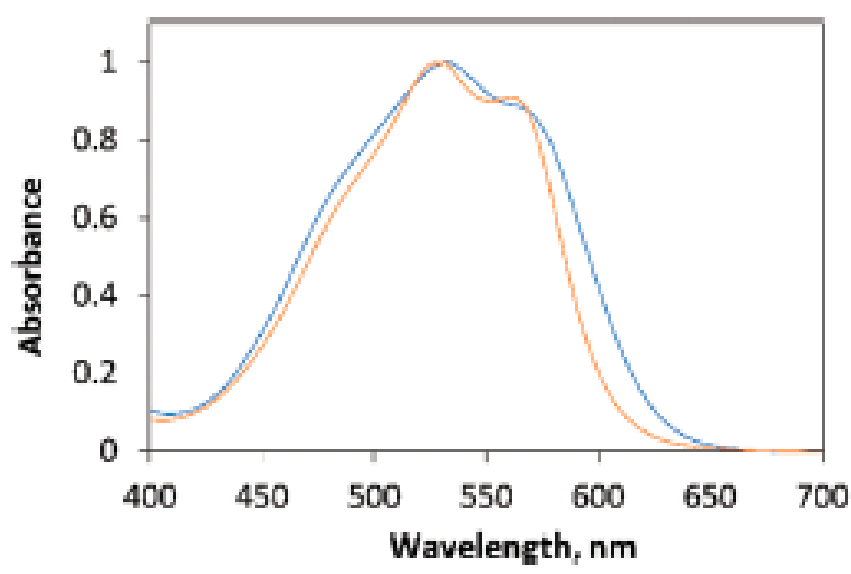

Figure 10. Left, two (a) and three (b) normalized spectral absorptions at $0.05 \mathrm{mM}$ concentration with $\mathrm{pH}$ of 7.0 . (c) Illustrative H-aggregates of TDPP with perfectly stacked face-to-face molecules orientation, rotating counterbalance to each other. Right, thin-films spectral absorptions of two (orange) and three (blue). Copyright, 2020, reproduced with permission from Reference [73].

\subsubsection{Solid-State Detection of Fluoride Ion}

Detection of fluoride anion $\mathrm{F}^{-}$capability with a synthesized probe 2a (Figure 11a,b) $)^{74}$ in solid-state, using test strips and glass thin-films, was explored by authors of Reference [74], through dipping of the strips and glass films in an arranged solutions of 2a in $(1 \mathrm{wt} \%)$ THF for strips. They also fabricated a glass thin-film via spin-coating (1500-2000 rpm) in a similar THF solvent with the same concentration (1 wt\%) that was allowed to dried in air. Probes $2 a$ and $3 a$ have an indigo and dark purple color, respectively. Subsequent dipping of $2 \mathrm{a}$ in solution of $\mathrm{F}^{-}\left(10^{-5} \mathrm{M}\right)$ ) changes its color from indigo to dark purple for both strips and glass films. The resulting dark purple color corresponds to the color of probe $3 a$ with no trimethylsilyl moiety, hence confirming $2 a$ to effectively detect $\mathrm{F}^{-}$in solid-state. This further indicates the imminent readiness of $2 \mathrm{a}$ for optical solid sensors application vividly. The detection mechanism of probe 2 a results from the strong electron-affinity of fluoride anion towards silicon which is obviously known; this interaction leads to the cleavage of the $\mathrm{Si}-\mathrm{C}$ bond of $2 \mathrm{a}$ and subsequently gives out probe 
3a another fluorescent molecule. It shows slight blue-shifted emission at 604 due to its interaction with $\mathrm{F}^{-}$, whereas an untreated 2a emits at $617 \mathrm{~nm}$. This experienced red-shifts emission is favored from the trimethylsilyl donating group.
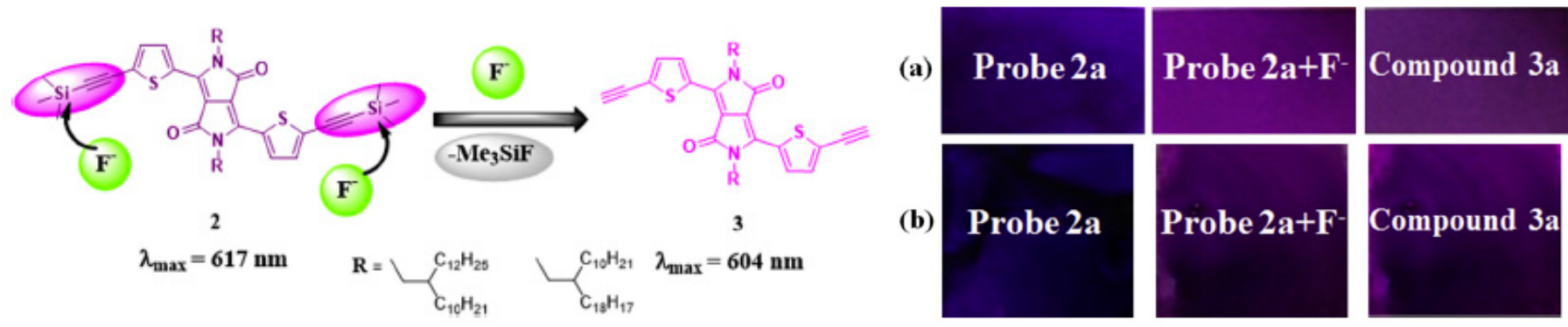

Figure 11. Designed method, molecule arrangement, and detection mechanism of fluoride anion by probe $2 \mathrm{a}$ and $2 \mathrm{~b}$. Pictures of probe $2 \mathrm{a}$, and then its interaction with $\mathrm{F}^{-}$and compound $3 \mathrm{a}$ on (a) filter test strips and (b) glass film. Copyright, 2014, reproduced with permission from Reference [74].

\section{Probes for Molecular/Bio-Imaging Applications}

Biomolecules are the famously known endogenous biomaterials secreted internally within an organism's biological system. Moreover, the biomaterials consist of proteins, carbohydrates, lipids and nucleic acids, in addition to primary and secondary metabolites along with other natural products. Generally, biomolecules are organic materials composed of oxygen, carbon, hydrogen and nitrogen as their major building block constituting about $96 \%$ weight of human body with trace amounts of other components, like biometals, in it. Biomolecules recognition is necessary because their chemical composition, biochemistry and interactions in the biological system reflect the physical and physiological functions (well-being or abnormality) scientifically. Therefore, such integral components are being detected and monitored concurrently, using different strategies in healthcare units.

Two-photon fluorescence microscopy (2PFM) presents a significantly deep tissue penetration $(>500 \mu \mathrm{m})$ [16], and hence it serves as a promising platform for biomolecules recognition, bio-imaging, therapy, etc., due to its noninvasiveness in biosensing of living cells and tissues. This results from the recently explored numerous reports of luminescent conjugated DPPs by studying their two-photon excitation/absorption behaviors in the biological system. The conjugated framework of this strongly acceptor core unit of DPP incorporating various donor groups were explored and have shown advancement in realizing an efficiently two-photon FPs. As a result, two-photon DPP FPs demonstrated valuable TPA cross-sectional values ranging (500-3000 GM) and above, hence indicating their potent deep tissue penetrability for imaging application to detect cancer/tumor diseases, and in some cases, they were even reported to have destroyed such cells.

\subsection{Probes for Biomolecules Detection}

As water-solubility remains challenging for biosensing and bio-imaging of DPP-based FPs, this restrict their practical application in biological systems. This motive lead Heyer et al. to developed a highly water-soluble bio-conjugate DPPs (Figure 12, the scheme) ${ }^{59}$ for BSA protein (50-100 mg/mL) from the exposed carboxylic acid linker that fluoresce strongly by using the strangely taurine-like disulfonated $(7,8)$ and tetrasulfonated $(9,10)$ linkers [59]. This study revealed that substituting phenyl with thienyl donor unit shifts the DPP's emission more to NIR around $600 \mathrm{~nm}$. The binding interactions of 7 and 8 with BSA protein indicate (1) aggregates composed of dye-BSA-bound molecules are not formed, (2) the DPP dyes are not perturbed by the protein environment and (3) possibly these dyes are embedded at the peripheral surface of the BSA proteins as depicted in (Figure 12, the spectra) ${ }^{59}$, because the absorption and emission of the freely unbound-dyes to that of bound-dyes displayed clearly good matching in aqueous media. Hence, it is worth noting that these optimized water-soluble organic fluorophores displayed valuable features that are rarely stated from the literature. Accordingly, certain 
stained amount of the BSA conjugates are studied through epifluorescence imaging microscopy (second-row Figure 12, left) ${ }^{59}$ by exploiting fluorescein isothiocyanate (FITC) and rhodamineB-specific for phenyl and thiophene DPPs respectively. Curiously, low limits of detection in the range of 80-300 molecules $/ \mu \mathrm{m}^{2}$ are observed for these BSA bio-conjugates. However, DPP-phenyl showed weaker emission and its limit of detection was higher by one order of magnitude compared to the DPP-thienyl conjugate. As mostly reported DPP FPs suffers the ACQ effect resulting from $\pi-\pi$ conjugation interactions along with their hydrophobic nature, these properties also greatly limit their bio-imaging applications. Tang et al. addressed this issue by developing two newly water-soluble probes DPP1 and DPP2, in that, BSA displayed a very significant PIFE after binding with DPP 1 and 2 materials through their terminally exposed carboxylic acid moieties [42]. This realized fluorescence turn-on event results from the existence of hydrophobic and hydrogen-bonding interactions between the synthesized dyes and BSA molecules through binding with the freely exposed carboxylic acids of these dyes, which consequently protect their fluorophore far away from quenchers like ions, thiols significantly thereby stabilizing the optimized DPP fluorophores. This is attributed to the obviously known serum albumen ability in binding and moving of different ligands to particular positions. Buffer solutions at $(\mathrm{pH}=7.4)$ of DPP 1 and 2 show pathetically weak emission. A low cytotoxic effect was experienced, as over $80 \%$ of viable cells endured $20 \mathrm{~h}$ incubation period after culturing even at $100 \mu \mathrm{m}$ in vitro when tested with HTC-116 cell via MTS assay, thus serving as an excellent luminous contrasting agent. These probes emit in NIR after $810 \mathrm{~nm}$ excitation wavelength using $2 \mathrm{PFM}$, hence functioning as valuable materials for nonlinear optical imaging application. This confocal fluorescence imaging revealed DPP1 and DPP2 as permeable probing agents to cells requiring no any permeabilizing agent. This was examined as a result of strongly observed emission from one-photon with the further observed large two-photon signal-to-noise ratio, which all originated from the PIFE of these dyes after binding to the cytoplasmic proteins (second-row Figure 12a-i) ${ }^{42}$.

Later, in 2018, the authors of Reference [28] tested ratiometric FPs for biorecognition of esterase enzymes by incorporating 4-bromomethyl-phenyl acetate with pyridinium-coated DPP fluorophore unit and a DPP-AM derivative was developed. This novel material exhibits strongly red emission at $655 \mathrm{~nm}$ due to ICT upon addition of esterase enzymes onto the DPP-AM, the ICT was destroyed as a result of acetyl ester bond cleavage through enzymatic hydrolysis, and thus changes the fluorescence spectral properties from red to yellow emission (blue-shifted $551 \mathrm{~nm}$ ) with $9.51 \times 10^{-5} \mathrm{U} / \mathrm{mL}$ limit of detection in vitro (Row-1, Figure 13 left) ${ }^{28}$. 

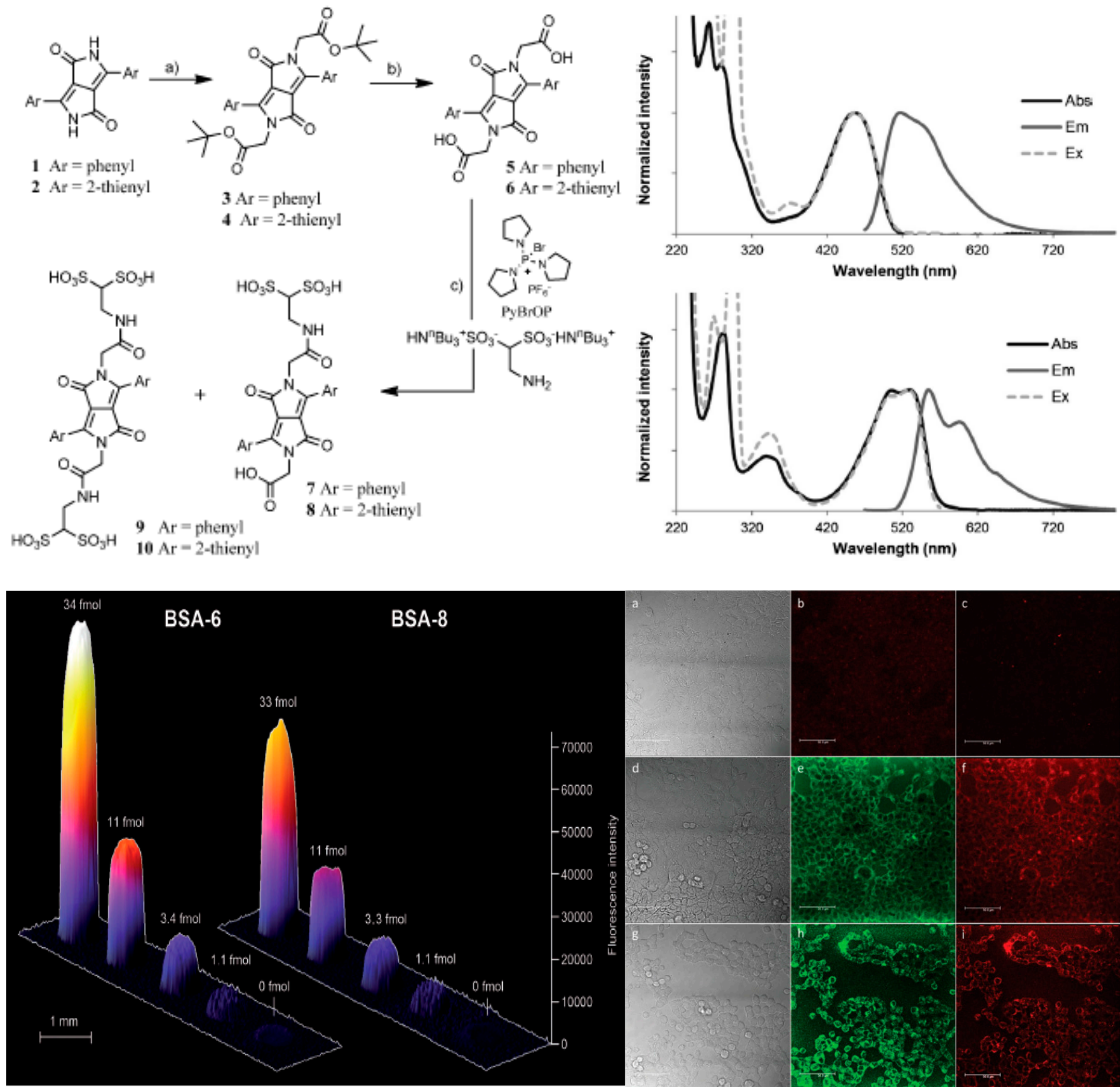

Figure 12. First row: left, synthesized molecules of water-soluble DPP-dyes. Right, absorption, fixed emissions at $550 \mathrm{~nm}$ for BSA-7 (top), $590 \mathrm{~nm}$ for BSA-8 (bottom) excited at $450 \mathrm{~nm}$, in PBS at $258^{\circ} \mathrm{C}$ (labeled using 15 equivalent at pH 7.0). Copyright, 2015 reproduced with permission from Reference [59]. Second row: left, a 3D colored epifluorescence microscopic imageries using specific rhodamine-B excited between 510 and $550 \mathrm{~nm}$ with emission wavelength of $590 \mathrm{~nm}$, spotted with individual certain quantities of BSA-6 and BSA-8 all leveled using 30 equivalent with pH 7.0 of these conjugates. Copyright, 2015 reproduced with permission from Reference [59]. Right, HCT-116 cell confocal fluorescence images of (controlled, (a-c)) cultured using $(25 \mu \mathrm{M},(\mathbf{d}-\mathbf{f}) ; 50 \mu \mathrm{M},(\mathbf{g}-\mathbf{i}))$ of DPP1. (a,d,g) DIC and $(\mathbf{b}, \mathbf{e}, \mathbf{h})$ are all 1PE (excited at $562 \mathrm{~nm})$, while (c,f,i) are 2PE excited at $810 \mathrm{~nm}$, in the absence of dye, cultured by using 25 and $50 \mu \mathrm{M}$ of DPP1, correspondingly. Copyright, 2017, reproduced with permission from Reference [42]. 

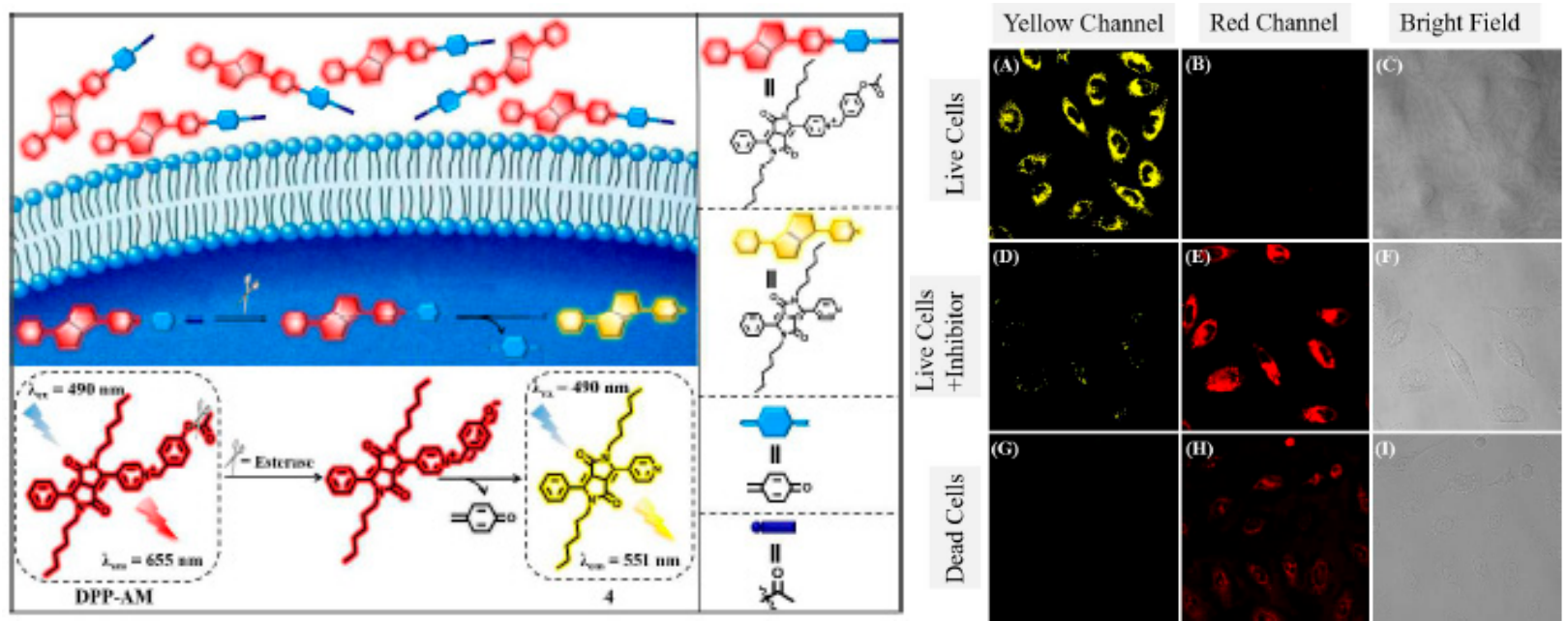

A

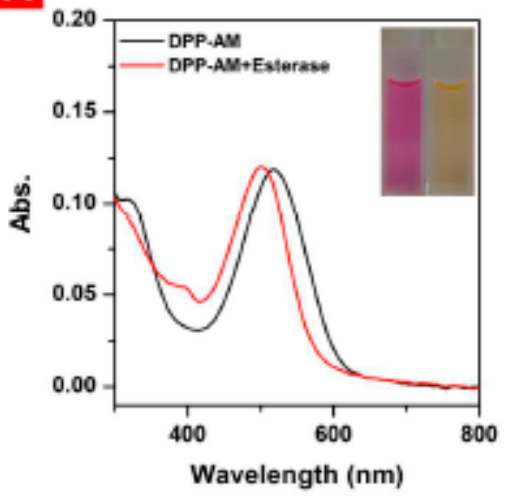

B

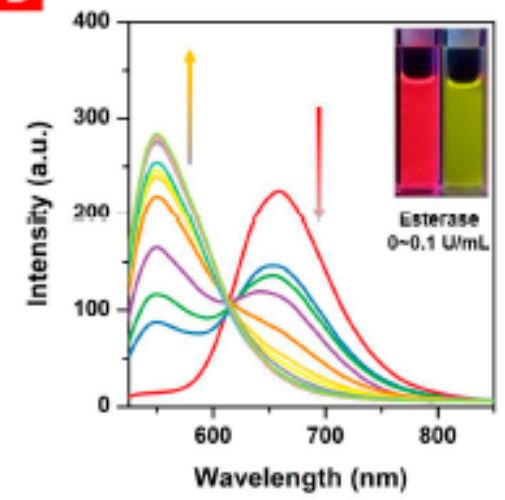

A

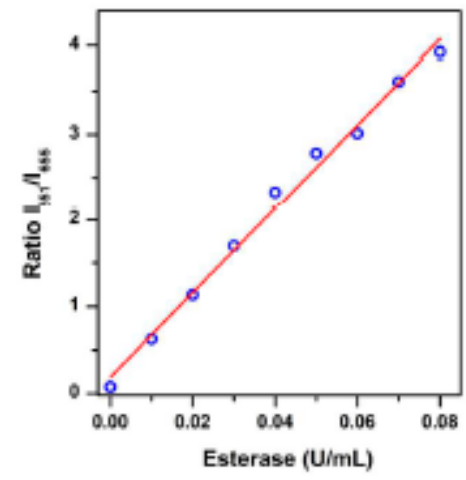

B

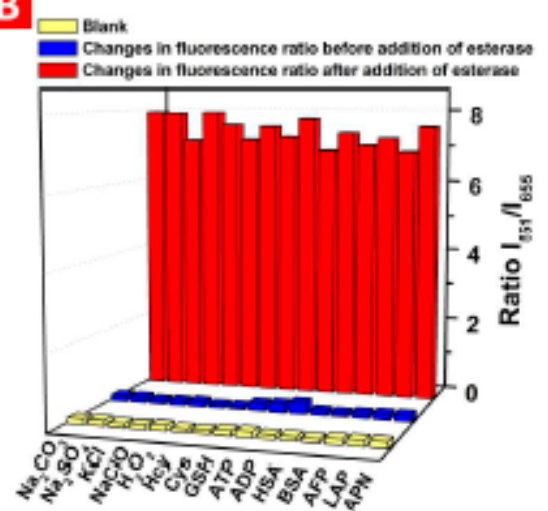

Figure 13. Row-1: left, DPP-AM molecule structure with its offered detection mechanism for esterase. Right, Live (A-C) and Dead (G-I) TPC1 cells fluorescent images marked using DPP-AM (10 $\mu$ M) for 45 min, while (D-F) pretreated TPC1 cells using AEBSF $(0.8 \mathrm{mM})$ for $60 \mathrm{~min}$ followed by additional $45 \mathrm{~min}$ incubation with DPP-AM (10 $\mu$ M). Yellow canal (525-585 nm), red canal (625-685 nm) excited at $490 \mathrm{~nm}$. Copyright, 2018 reproduced with permission from Reference [28]. Row-2: (A) DPP-AM $(10 \mu \mathrm{M})$ absorption spectral before (left) and after Esterase $(0.1 \mathrm{U} / \mathrm{mL})$ addition (right) in DMSO/PBS (DMSO: $\mathrm{PBS}=2: 8, v / v, \mathrm{pH}=7.4$ ) for $20 \mathrm{~min}$. at $37^{\circ} \mathrm{C}$. Inset: photo displaying the optical DPP-AM colors while testing under visible light. (B) DPP-AM $(10 \mu \mathrm{M})$ with $0-0.1 \mathrm{U} / \mathrm{mL}$ esterase fluorescence spectral emission for $20 \mathrm{~min}$. at $37^{\circ} \mathrm{C}$. Inset: photo displaying the optical DPP-AM fluorescent colors while testing under $365 \mathrm{~nm}$ UV light, excited at $490 \mathrm{~nm}$. Row-3: (A) line graph of $\mathrm{R}$ against Esterase concentration in the range of 0-0.08 U/mL, excited at $490 \mathrm{~nm}$. (B) Fluorescence response of DPP-AM $(10 \mu \mathrm{M})$ to various analytes at certain solution or mixture concentrations excited at $490 \mathrm{~nm}$. Copyright, 2018 reproduced with permission from Reference [28]. 
Even more interesting, is the fact that DPP-AM clearly differentiate live from dead cells, where its yellow emission designates live cells existence while red emission signifies the existence of dead cells (Row-1, Figure 13, right) ${ }^{28}$. This findings, affirms that DPP-AM possess substantial capability to assess the activity of esterase enzymes as great esterase activity was experienced for live cells and such activity was insufficiently discovered for dead or wounded cells. The spectroscopic response of resulting derivative (DPP-AM) exhibits a $520 \mathrm{~nm}$ absorption (blueshift) clearly seen with naked eyes (Row-2, Figure 13A,B) ${ }^{28}$. Accordingly, its emission spectra show red emission $655 \mathrm{~nm} F Q Y=0.41$, without esterase, and a subsequent addition of esterase makes it (551 nm FQY = 0.67) blue-shift with an enhanced FQY. In addition, DPP-AM showed a sensitive and selective esterase detection in the presence of numerous interfering analytes of inorganic salts, reactive oxygen species, amino acids and different proteins, thus accentuating its effectiveness in monitoring of esterase enzymes (Row-3, Figure 13A,B) ${ }^{28}$.

\subsection{Probes for Photodynamic/Photothermal Therapy}

Fluorescent DPPs shows therapeutic/theranostic functions as well, as various DPPbased FPs proves promising to trace biomarkers like mitochondria staining, detect cancer/tumor tissues via numerous approach such as bio-imaging using photoacoustic imaging (PAI), photodynamic therapy (PDT), photothermal therapy (PTT) and lots more. Presently most widely used theranostic systems largely shows much dependence on fluorescence, yet efficient light penetration depth and background autofluorescence limits their investigative accurateness. The PAI, a spatially distributive with great persistence technique and profound tissue penetrating bio-imaging technology, can effectively realize a 3D tumor imaging in clinical research for biomedical applications. As obviously known, PDT and PTT combination in one strategic platform generate problems of time elongation therapy with systemic side-effects to patients as varied excited wavelengths are needed to generate the required energy (ROS/heat) for this synergistic system to work efficiently. Therefore, developing a new and single module healing agents with NIR absorption to grasp PAI-guided PDT/PTT which can significantly boost efficient, safe and accurate therapy of cancer is highly commendable. For this reason, Cai et al. offered an innovative single component DPP-based DPP-TPA organic nanoparticles (NPs) with typical donor unit of triphenylamine (TPA) for cancer therapy [62], and have shown a vivid tumor destruction in mice via the PAI-guided synergistic effect of PDT/PTT in vivo at even lower dosage of $(0.2 \mathrm{mg} / \mathrm{kg})$ (Row-1, Figure $14 \mathrm{c})^{62}$, after illuminated at $(660 \mathrm{~nm}$ $1.0 \mathrm{~W} / \mathrm{cm}^{2}$ ) with laser through enhanced permeability and retention (EPR) effect with this novel material. Accordingly, in vivo and in vitro investigation exposed this single component to have generated ROS and heat, required parameters for efficient theranostic analysis for this synergistic PDT/PTT, with very fast tumor targeting within $2 \mathrm{~h}$ (Row-2, Figure $14 a, b)^{62}$, thus, aiding significant inhibition of tumor growth (Row-2, Figure 14c,d) ${ }^{62}$. Moreover, the material displayed appreciably great photothermal conversion efficacy of $(\eta=34.5 \%)$ with admirable singlet oxygen $\left({ }^{1} \mathrm{O}_{2}\right)$ production $\left(\Phi_{\Delta}=33.6 \%\right)$ under same laser illuminated condition. Additionally, ex vivo histology study shows no visible tissue damage or any noticeable side-effect of the examined main body organs on hematoxylin and eosin (H\&E) spotted slices for heart, liver, spleen, lungs and kidney, hence, implying the safe and efficacy of DPP-TPA NPs with its capability to destroy cancerous tumor cells as clearly seen below. 
(a)

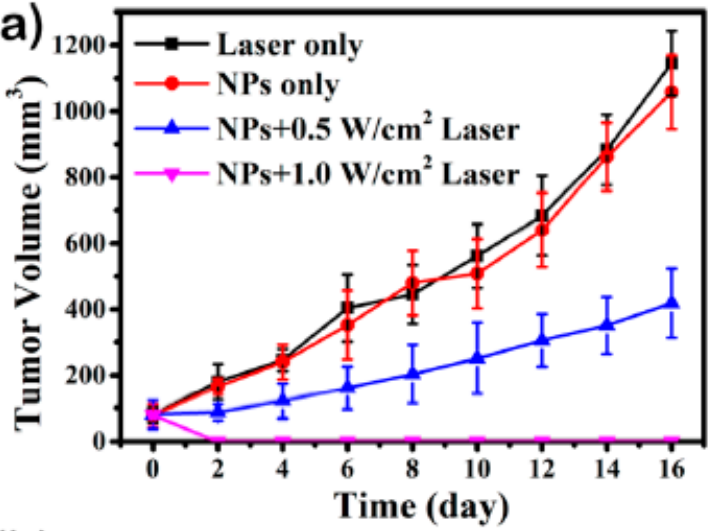

(b)

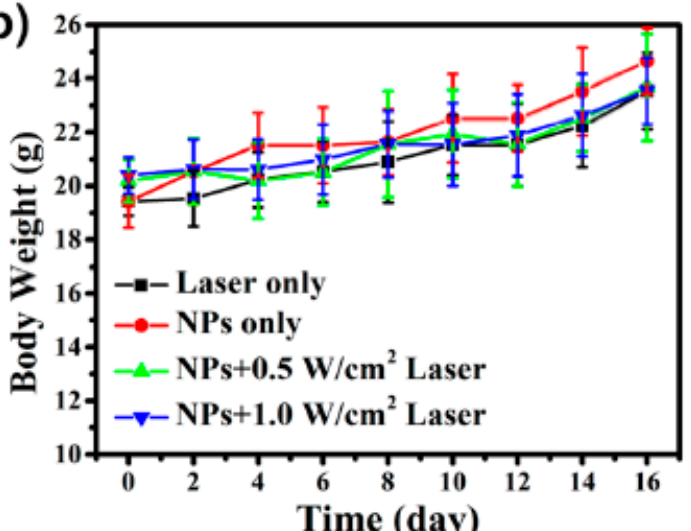

(c)
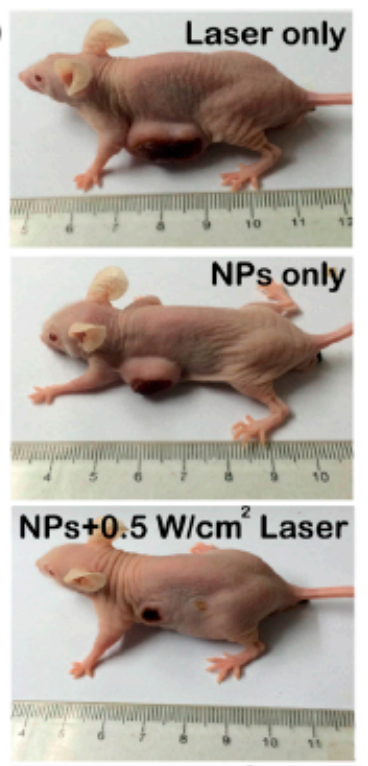

NPs+1.0W/cm Laser

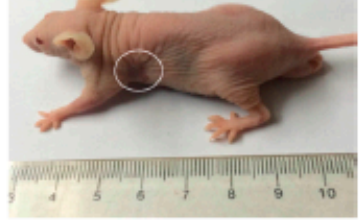

$\mathrm{h}$

(a)

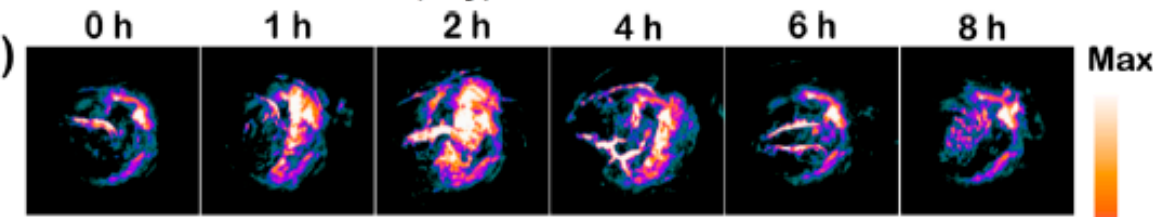

(b)

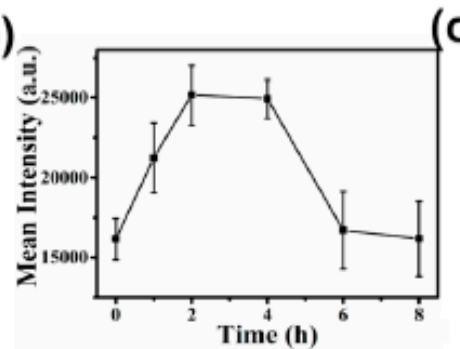

(c)

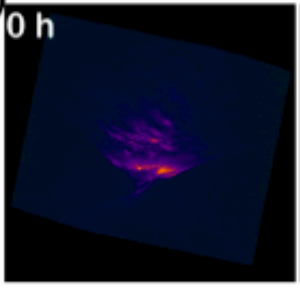

$2 \mathrm{~h}$
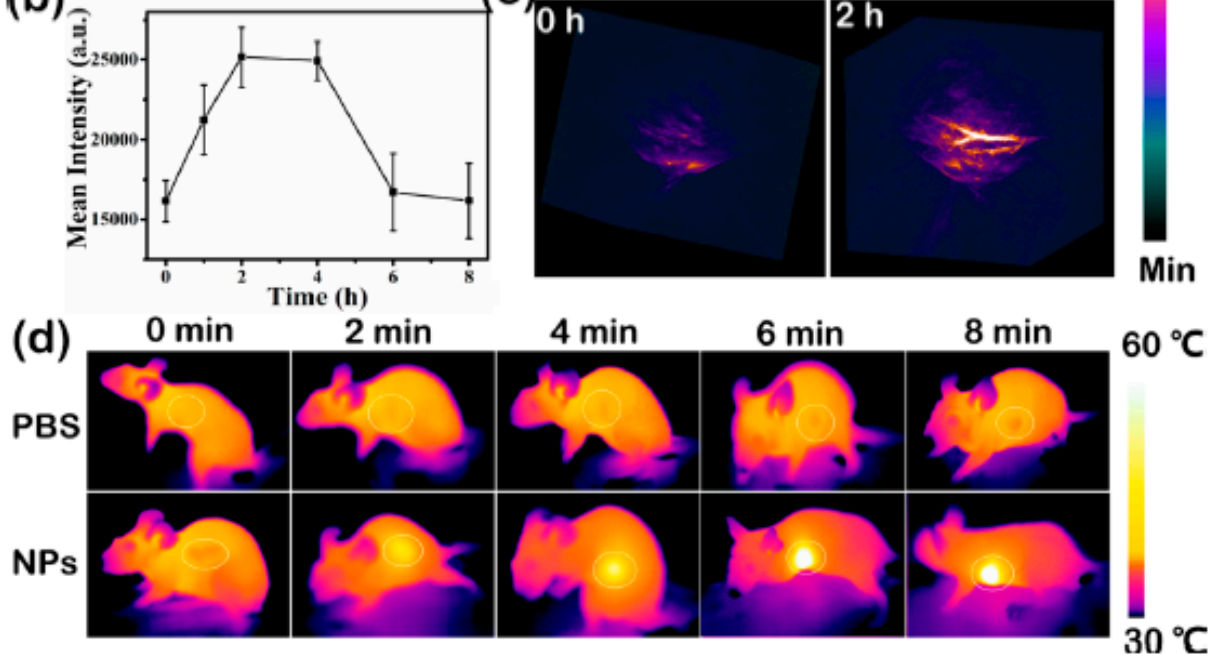

Figure 14. Row-1: (a) comparing tumor sizes with time for different remedy assemblies. (b) Variations in body mass with time for different remedy assemblies. (c) Mice images when curing for 16 days. Copyright, 2017, reproduced with permission from Reference [62]. Row-2: (a) malignant tumor sites PAI after vaccinating cancerous mice with DPP-TPA NPs $(0.2 \mathrm{mg} / \mathrm{kg}, \mathrm{n}=4)$ intravenously. (b) In vivo PA intensity matching at various time interval. (c) Three-dimensional malignant tumor positions PAI for 0 and $2 \mathrm{~h}$ post-treatment. (d) Infrared photograph positions of vaccinated cancerous mice with PBS and DPP-TPA NPs illuminated at varied time intervals for $2 \mathrm{~h}$. Copyright, 2017 reproduced with permission from Reference [62]. 
Similar collaborative research groups later, in 2019, described another novel (DPPBDT) a small molecule FP (Row-1, Figure 15$)^{12}$ with exceptionally pronounced PDT and PTT therapeutic performance with a featured absorption and emission ranging $(650-1700 \mathrm{~nm})$ for NIR-I and NIR-II windows respectively [12]. This novel organic small molecule was engulf with an amphiphilic DSFE-mPEG5000 which successfully resulted to a developed multi-functional phototheranostic nano-agents that were found soluble in water, exceptionally biocompatible with high photostability. As PDT and PTT efficacy arouses greatly from hypoxia microenvironment near the tumor cells along with attained thermal-resistance lingering cancer cells characteristics, which cripples the therapy efficiency with either PDT or PTT. Therefore, of significant advantage is the dual-modal imaging of PDT/PTT as an alternative platform for circumventing these mentioned challenges with positive outcome. Cell viability studies (Row-2, Figure $15 a, b)^{12}$ for separately stained live and dead cells in dark environment with DPPBDT displayed wide green emission after incubating Hela cells (Row-2, Figure 15c) ${ }^{12}$, thus proving the anticancer efficacy of this novel material, as a negligible cytotoxic effect was exhibited by the DPP-BDT NPs. Aiding from the dual-modal imaging of NIR-II fluorescence/photoacoustic (Row-3, Figure 15a,b) ${ }^{12}$ steered from the combined PTT/PDT treatment, outstanding capabilities for tumor cells destruction were observed (Row-4, Figure $15 \mathrm{a}-\mathrm{d})^{12}$ by this nano-agents through in vitro and in vivo analyses after laser irradiation with a single wavelength. Accordingly, strongly red emission was vividly shown, thus, strengthened the material's NPs high light cytotoxic effect for cancer therapy. Furthermore, the DPP-BDT NIR-II FQY was found to be $0.52 \%$ peaked at $980 \mathrm{~nm}$. Moreover, no visible tissue damage was observed from investigative study of the (H\&E) spotted slices for heart, liver, spleen, lungs and kidney as well, therefore again strengthening the promising biomedical therapy of this novel DPP-BDT NPs innoxious to these delicate human biological tissues. Thus, this finding indicates potentials of DPPs NPs as effective healing agents for cancer/tumor destruction via PDT/PTT with negligible side-effect.

The photosensitizing and photothermal effects of DPPs in combating tumors/cancer therapy (phototheranostics) were also recently reviewed [2,75], along with their ease of modification, exceptional photophysical features (high FQYs, superb thermo-stability and photostability). 

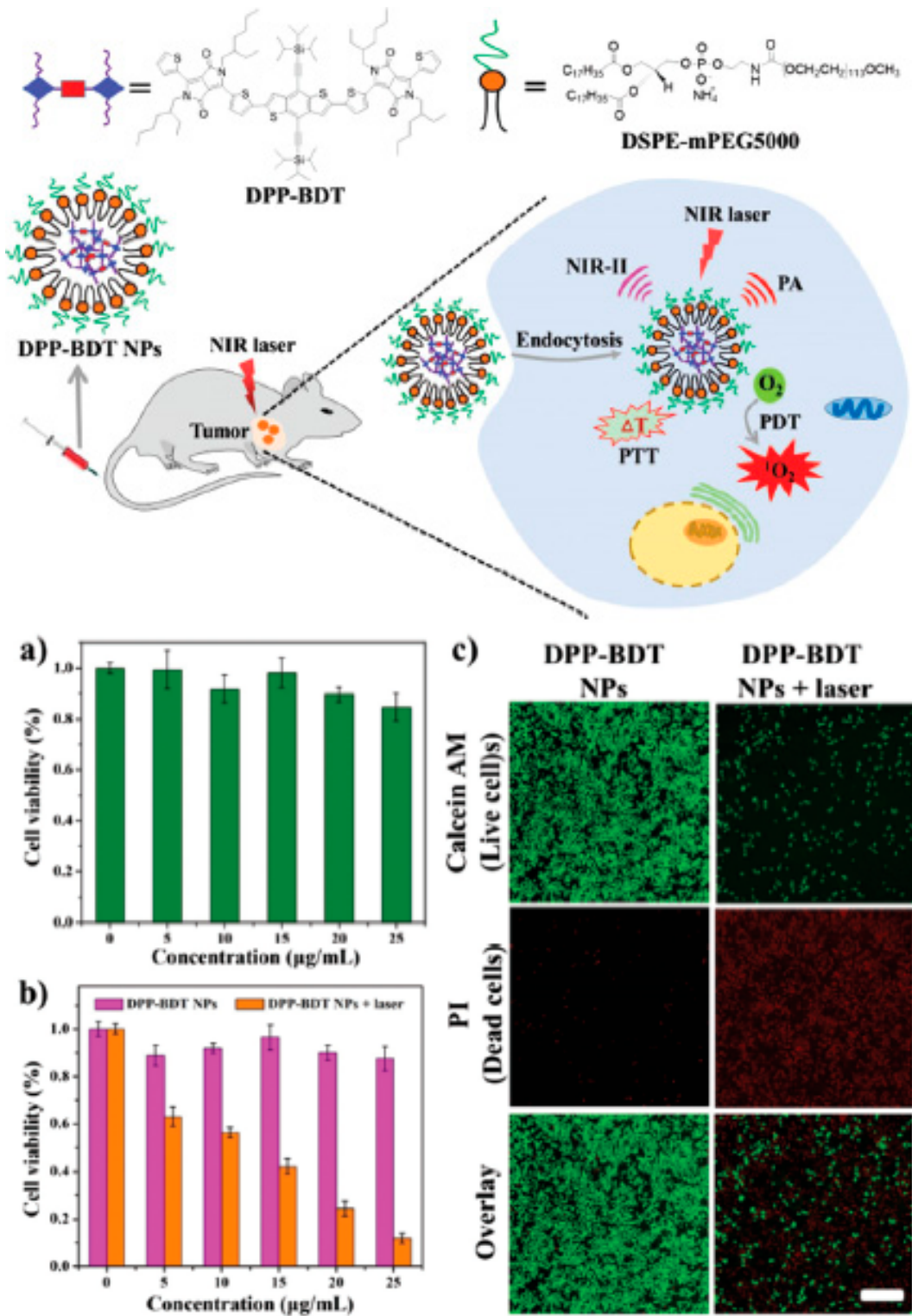

a)
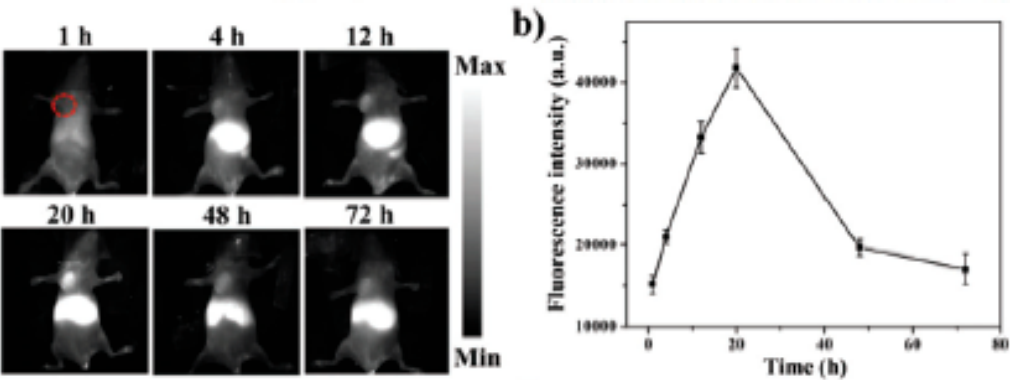

c)

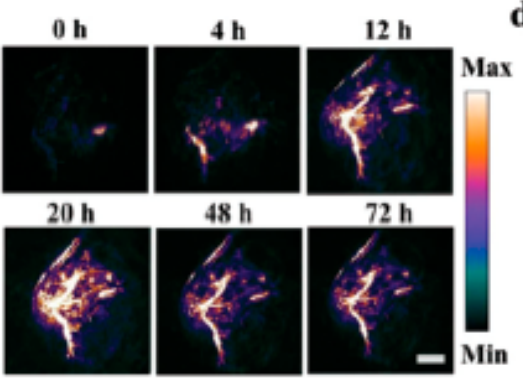

d)

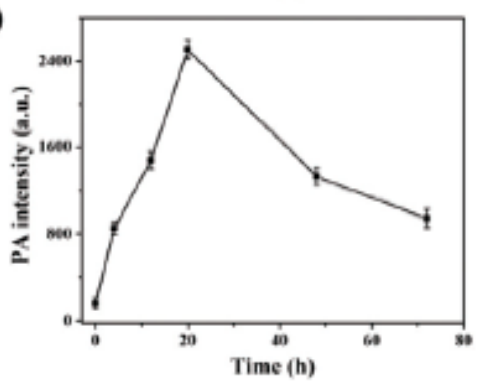

Figure 15. Cont. 
a)

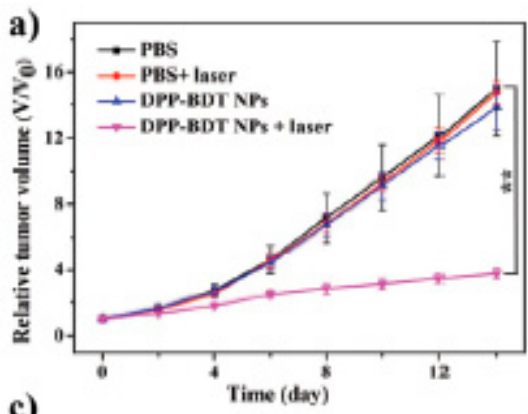

c)

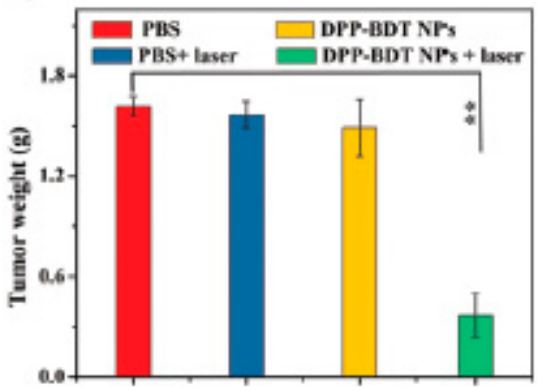

b)

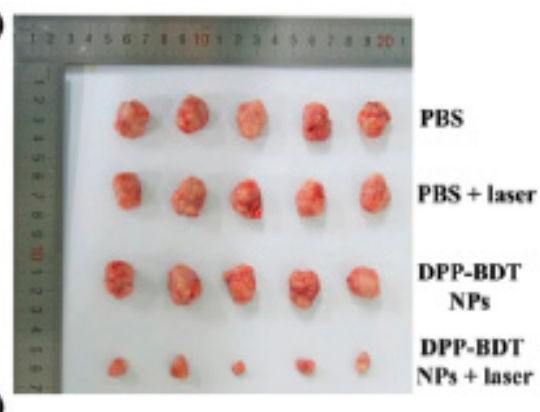

d)

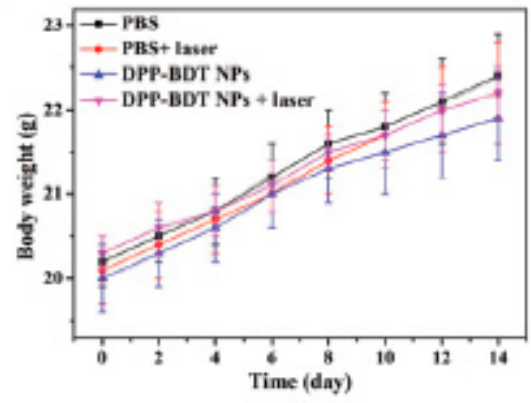

Figure 15. Row-1: DPP phototheranostics molecule used for NIR-II fluorescence/PAI along with immediate PDT/PTT combined effect remedy Copyright, 2019 reproduced with permission from Reference [12]. Row-2: (a) DPP-BDT NPs compatibility tests on NIH-3T3 purely normal cells. (b) Cytotoxic effect assays (c) Live and dead HeLa cell assays cured with and without DPP-BDT NPs irradiation. Copyright, 2019 reproduced with permission from Reference [12]. Row-3: (a) fluorescence images of NIR-II, (b) Variations in NIR-II signal intensity, (c) PAI (d) PA signal deviations of intravenously injected DPP-BDT NP to HeLa tumor-bearing mice. Row-4: (a) growth curves for differently treated malignant tumor cells. (b) Malignant tumor photographs and (c) weights of differently treated malignant tumors during treatment. (d) Body-weight changes of differently treated groups. Copyright, 2019 reproduced with permission from Reference [12].

\subsection{Prostate Cancer Probes}

In another development (2019), by the authors of Reference [76], DPP FPs also proved to be promising towards prostate cancer tissues recognition and capable of discriminating between live and dead tissues in vivo; this was observed following the exploration of chained DPP-based AND logic FPs (DPPC2, LysoDPPC2, LysoDPPC3 and LysoDPPC4), prepared by incorporating an aliphatic morpholine functionality (a pH-responsive indicator and lysosometargeting subunit) (Figure 16 scheme $)^{76}$. It was found that protonating these probes with $\mathrm{Zn}^{2+}$ suppresses the single PET channel, which leads to little fluorescence intensity increment. Conversely, upon blockage of all PET channels, the fluorescence intensity was restored fully under an acidic condition and high $\mathrm{Zn}^{2+}$ concentration (Figure 16 intensity signal) ${ }^{76}$, in that LysoDPP-C4 FP demonstrated a significant low $\mathrm{pH}$ detection and lysosomal biosensing of $\mathrm{Zn}^{2+}$ in live cells accompanied with a pronounced increased in fluorescence intensity. This was attested via fluorescence imaging microscopy of nude mice, where the cancer prostate and seminal vesicles are significantly identified by strong green emission, using the LysoDPPC4 probe (Figure 16c) ${ }^{76}$ consistent with the literature for high $\mathrm{Zn}^{2+}$ content observed by these organs, with a corresponding negligible cytotoxicity signal for the healthy cells. Essentially important is the fact that this FP LysoDPPC4 proves effective for discriminating between live prostate and cancer prostate cells through in vivo and histology study of prostate, seminal vesicles, liver, kidney, brain and muscles (Figure $16 \mathrm{e})^{76}$. 

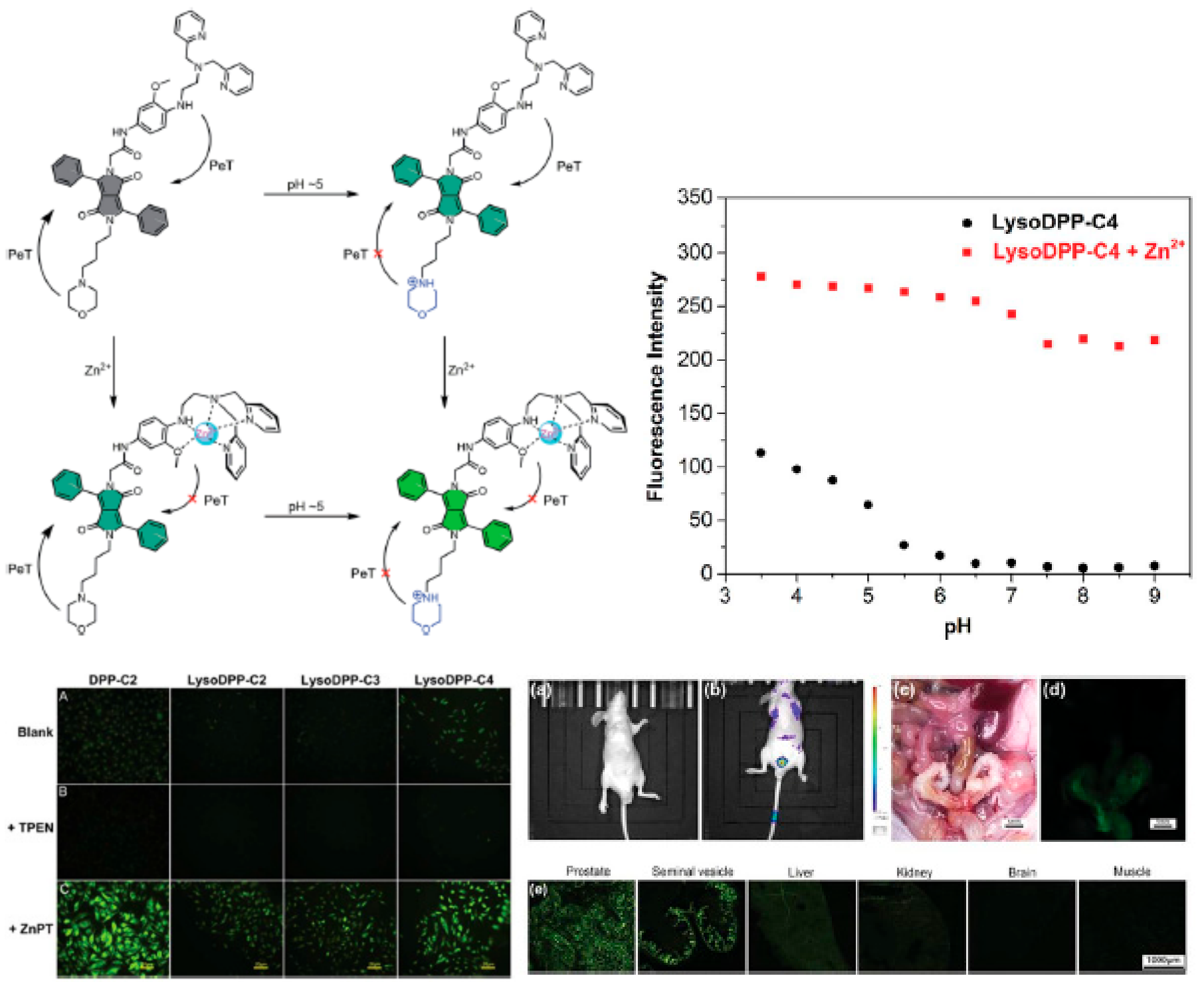

Figure 16. First row: left, mechanistic fluorescence turn-on process permitting a concomitant low $\mathrm{pH}$ AND $\mathrm{Zn}^{2+}$ recognition (weakly gray with strongly green fluorescence signal intensities). Right, LysoDPP-C4 (1 mM) Fluorescence intensity signal with its zinc-complex solutions at different $\mathrm{pH}$ excited at $430 \mathrm{~nm}$ with emission wavelength of $515 \mathrm{~nm}$. Second row: left, HeLa cells fluorescence images. Row-A, incubated cells, using $10 \mathrm{mM}$ of the used probe, at $24 \mathrm{~h}$; Row-B, incubated cells, using $10 \mathrm{mM}$ of the used probe, at $24 \mathrm{~h}$, which was further nurtured for $10 \mathrm{~min}$, using $50 \mathrm{mM}$ TPEN; Row-C, incubated cells, using $10 \mathrm{mM}$ of the used probe, at $24 \mathrm{~h}$, which was later nurtured for $10 \mathrm{~min}$ by mixing with $60 \mathrm{mM}$ ZnPT. Right, in vivo describing entirely the healthy nude-mice visual images via an epifluorescence microscopy prior to vaccination (a) after vaccinated intravenously via the tail for $15 \mathrm{~min}$ (b) with LysoDPP-C4 $500 \mathrm{mg}$ per single mouse, the prostate-area fluoresce strongly after vaccinating. After sacrificing and dissection, prostate and seminal vesicles displayed brightly green emission; however, (c,d) other viscera and skeletal muscles were non-emissive (dark). (e) Prostate, seminal vesicles, liver, kidney, brain and adjacent muscle tissue ex vivo fluorescence images gotten from the vaccinated mouse with LysoDPP-C4. Copyright, 2019, reproduced with permission from Reference [76].

Accordingly, due to zinc being a reliable prostate cancer biomarker, meanwhile it is obviously known that prostate cancer tumors possessed low zinc concentration in relation to the normal healthy prostate tissues. For this reason, in the same year, Fu et al. presented other FP coded DPP-C2 (Scheme 1b) ${ }^{49}$ above for the same purpose to investigate its ability to recognize prostate cancer tumors via an endogenous $\mathrm{Zn}^{2+}$ biosensing platform (an alternate approach). The study revealed that, DPP-C2 has negligible cytotoxicity in vitro and in vivo for both tumor (DU145 and PC3) and healthy (RWPE-1) prostate tissues (first 
row, Figure $17 \mathrm{C})^{49}$, in that healthy tissues fluoresce strongly red which increases upon culturing in zinc-supplement medium $\left(\mathrm{ZnSO}_{4}\right)$ (second row, Figure $\left.17 \mathrm{C}, \mathrm{D}\right)^{49}$, nevertheless the tumor tissues remained unaffected. Moreover, subsequent intravenous administration of DPP-C2 molecule to a cancerous nude-mice through the tail, and then examined the organism via fluorescence imaging stereomicroscopy. To attest the precise signal region, the mice was as well dissected and its viscera images demonstrated a very strong red fluorescence signal by the seminal vesicles (second row, Figure 17D,H) ${ }^{49}$, while the bladder, liver, kidney, spleen and skeletal muscles were (dark) non-fluorescing [49]. Tumor tissues displayed weak emission contrary to the nearby dark regions (skeletal muscles, abdominal and pelvic) which are similar with the tumor-free mice. Such observations are further proved with the observed higher zinc content in (DU145 and PC3) tumor cells compared to liver, kidney and skeletal muscles which were found to be lower, and conversely, the prostate possessed greatest zinc content, then next the seminal vesicles (second row Figure 17I) ${ }^{49}$, tested using inductively coupled plasma mass spectrometry (ICP-MS).
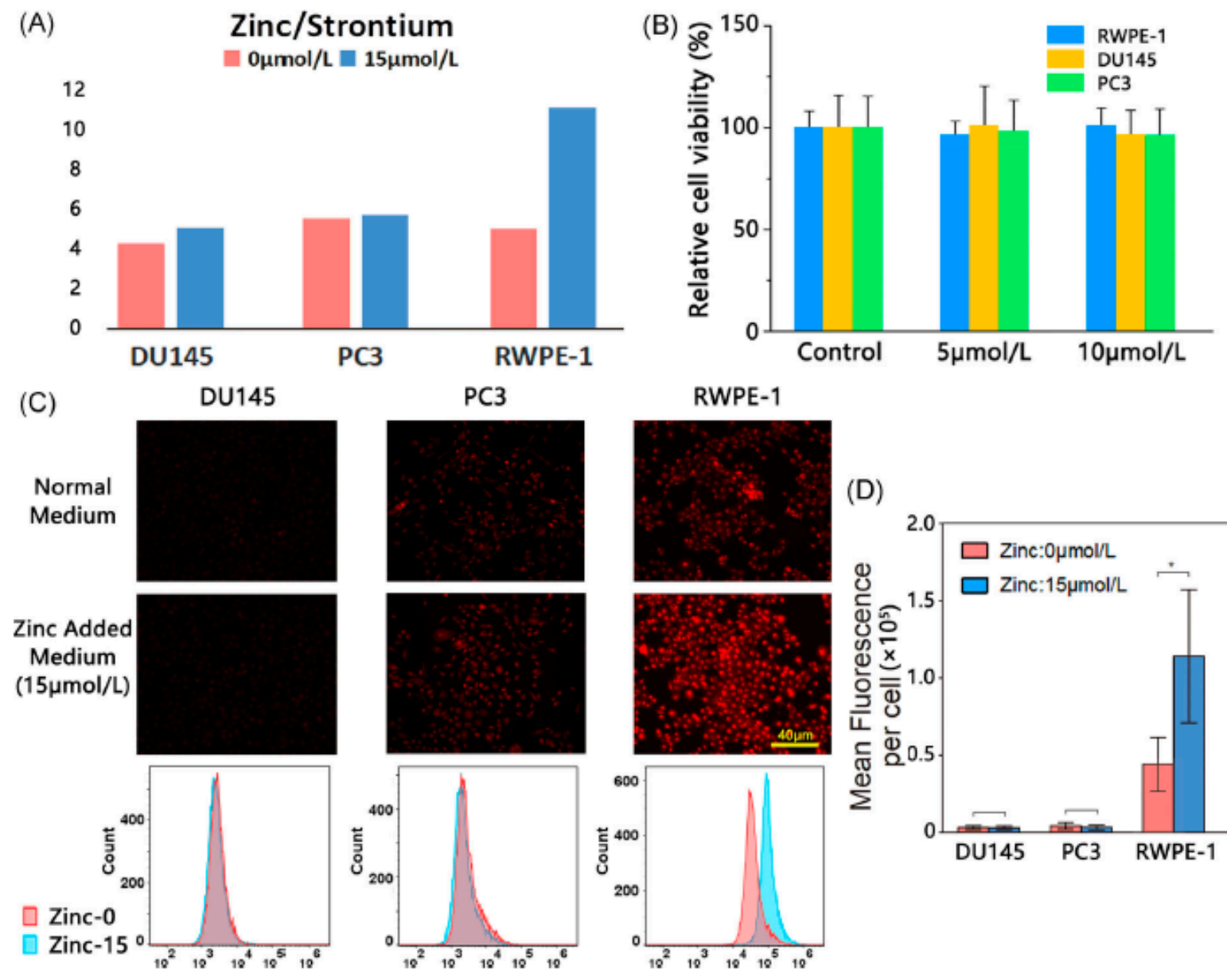

(D)

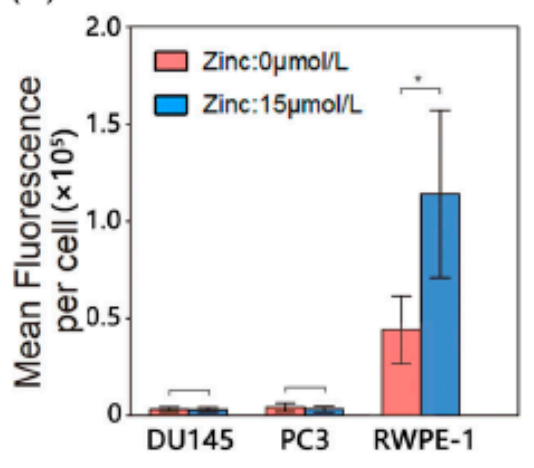

Figure 17. Cont. 


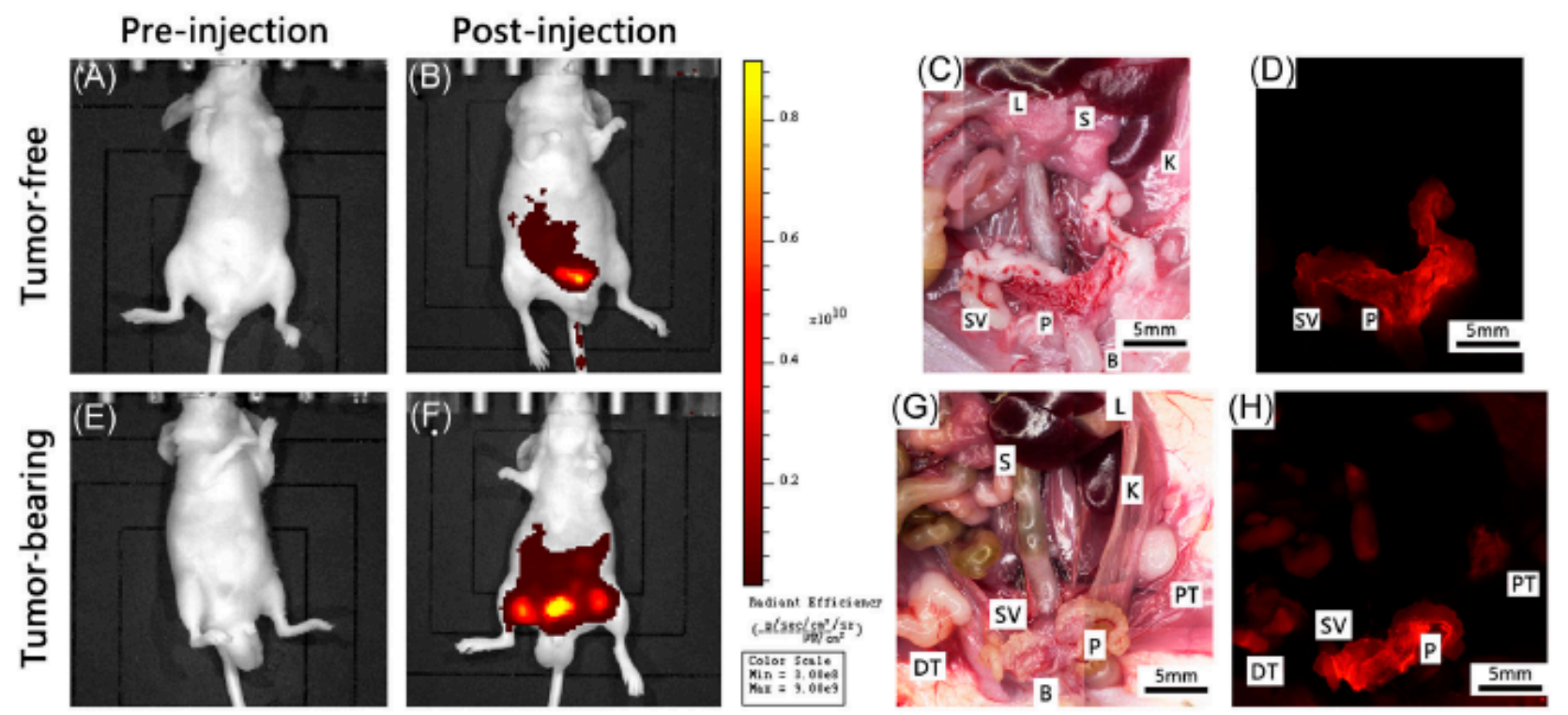

(I) 140 Zinc/Strontium

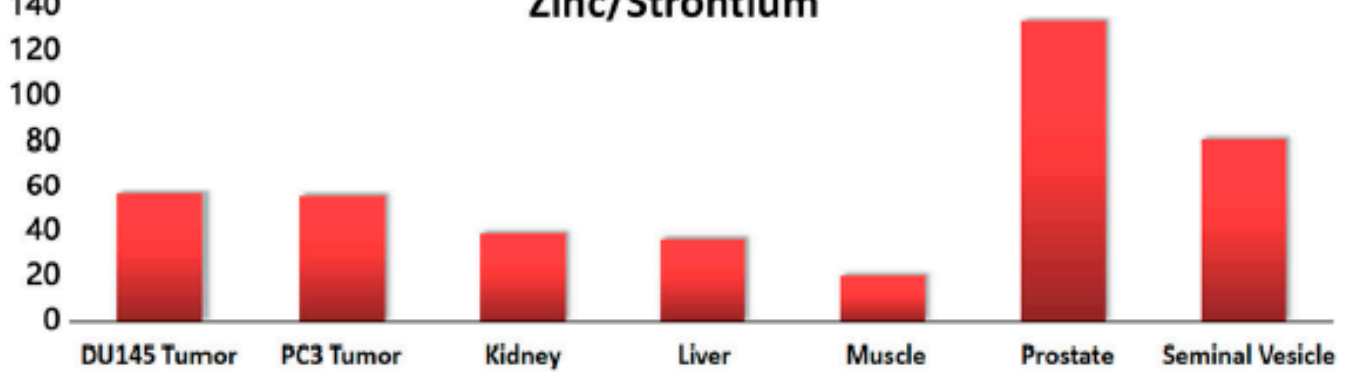

Figure 17. First row: analyses in vitro. (A) comparative intracellular zinc content (zinc and strontium) of DU145, PC3 and RWPE-1 cells in the presence and absence of $\left(\mathrm{ZnSO}_{4}\right)$ culturing agent. Pink and blue bars symbolize the cultured cells at 0 and $15 \mu \mathrm{mol} / \mathrm{L}\left(\mathrm{ZnSO}_{4}\right)$ concentrated media respectively. (B) flow cytometry raw histogram with the cells quantification (C) Examined tumorous and healthy cells fluorescent microscopic images in the presence and absence of $\left(\mathrm{ZnSO}_{4}\right)$ culturing agent (D) significant rise in fluorescence of healthy cells (RWPE-1) after culturing in $\left(\mathrm{ZnSO}_{4}\right)$ medium. Second row: Analyses in vivo. (A) Prior to injecting healthy nude-mice, (B) $20 \mathrm{~min}$ later, (C,D) after injecting DPP-C2 to the cancer-prostate region, strong red fluorescence signals appeared. (E) Prior to injecting healthy nude-mice, (F) 20 min later, $(\mathbf{G}, \mathbf{H})$ after injecting DPP-C2 to both cancer-prostate region and bilateral groins, prostate along with seminal vesicles fluoresce strongly. Moreover, the tumorous DU145 (DT) and PC3 (PT) cells fluoresce weakly. (I) ICP-MS data of various tissues studies have shown prostate to possess highest zinc content, then seminal vesicles, but for PT and DT tumors, it was a little higher in relation to liver (L), kidney (K) and skeletal muscles. Copyright, 2019, reproduced with permission from Reference [49].

\subsection{Mitochondria Staining Probes}

Despite the appreciable bio-imaging significance been exhibited by the few DPPbased FPs, yet such probes for specific accumulation and staining of mitochondria are rarely observed with DPPs, and are solely charged-based probes (incorporated moieties) like triphenylphosphonium or pyridinium. Nevertheless, such FPs shows substantial value towards staining of mitochondria. This follows the establishment by Grzybowski et al. of water-soluble DPP-based FPs of furan, thiophene and benzene ring-donors with each derivative bearing an imidazolium cationic unit. These probes demonstrated specific marking of mitochondria in alive cells via a 2PFM [41]. As crystal analysis of DPP flanked to 2-furyl or thienyl revealed an approximate angle lying between 0 and $10^{\circ}$, which provides better conjugation to the DPP chromophore compared to phenyl ring being twisted by about $30^{\circ}$, it follows that incorporating 2-furyl/2-benzofuryl substituted-amide onto the DPP core to have boosted the TPA cross-sectional values above 3000 GM with 1850 GM as two-photon brightness. As mostly reported DPP chromophores for 2PFM being non-polar, 
it follows that charged derivatives bearing imidazolium cation were proposed to selectively stained mitochondria, in that DPPs coded $3 \mathrm{~b}$ and $3 \mathrm{c}$ (Scheme $2 \mathrm{~A})^{41}$ above revealed a TPA values of 1930 and $220 \mathrm{GM}$, respectively. Subsequent optimization of $3 \mathrm{~b}$ and $3 \mathrm{c}$ yielded compounds $4 \mathrm{~b}$ and $4 \mathrm{c}$ with significantly enhanced TPA values of 4300 and $3400 \mathrm{GM}$, correspondingly, in DMSO (first row, Figure $18 \mathrm{a}-\mathrm{c}$, left) ${ }^{41}$. Confocal fluorescence imaging study with (Mitotracker green) depicted DPPs $4 \mathrm{a}-\mathrm{c}$ to have penetrated live cells cellular membrane (first-row images $18 \mathrm{a}-\mathrm{c}$, right) ${ }^{41}$ with specific localization of mitochondria at both micro- and sub-micromolar levels. Hence, this indicated that substituting phenyl with furyl and/or thienyl rings leads to a 10-fold TPA cross-sectional enhancement. Cell viability revealed that $10 \mu \mathrm{m}$ concentration of $4 \mathrm{a}-\mathrm{c}$ probes does not affect viable cells (a 10 -fold concentration rise which is greater than the one used for such microscopic analyses). However, insignificant cytotoxicity was shown upon incubating Hela cells with 4a-c probes at $100 \mu \mathrm{m}$ concentration (far above concentrations being commonly used for microscopic analysis); thus, such observed cytotoxicity is of no significance biologically.

a)

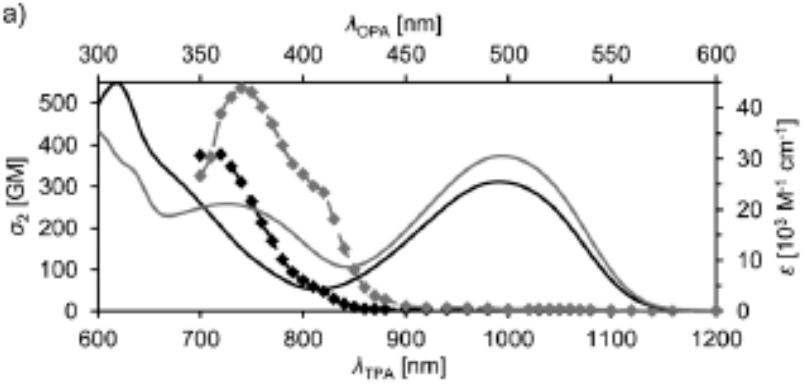

b)

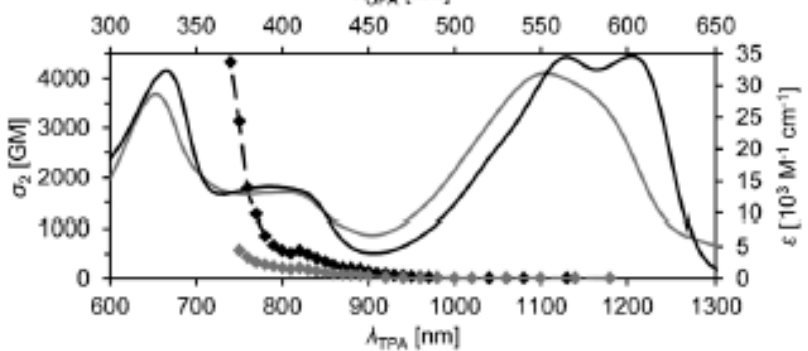

c)

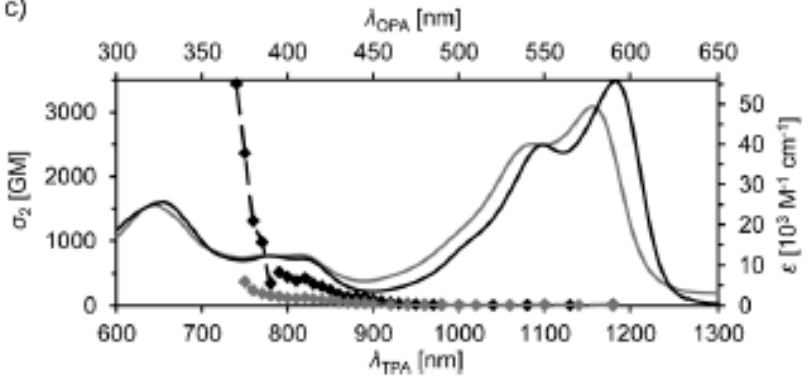

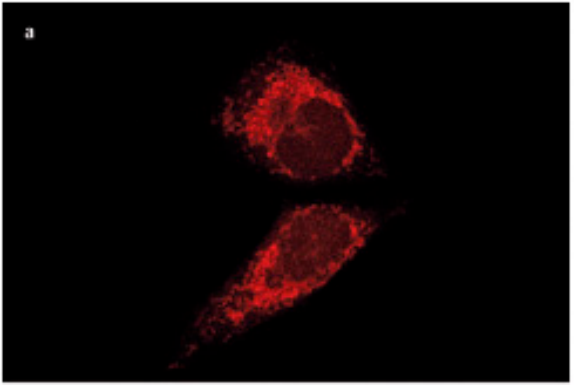
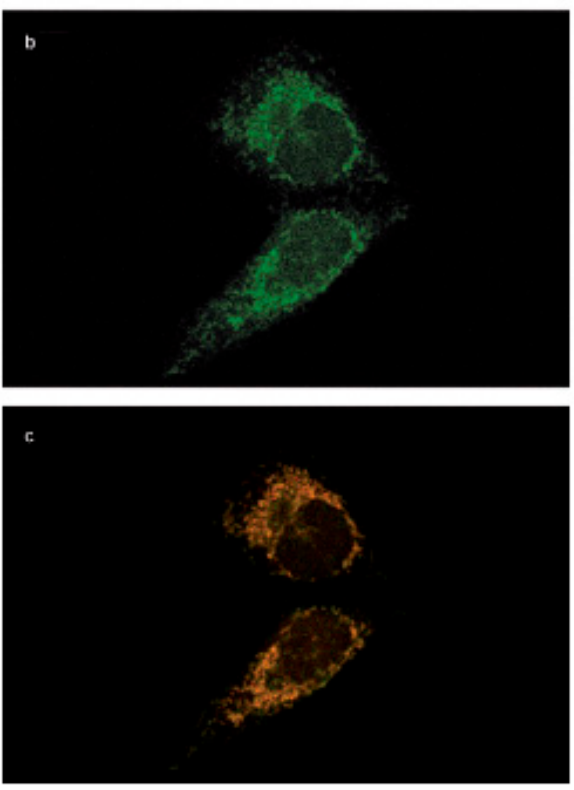

Figure 18. Cont. 

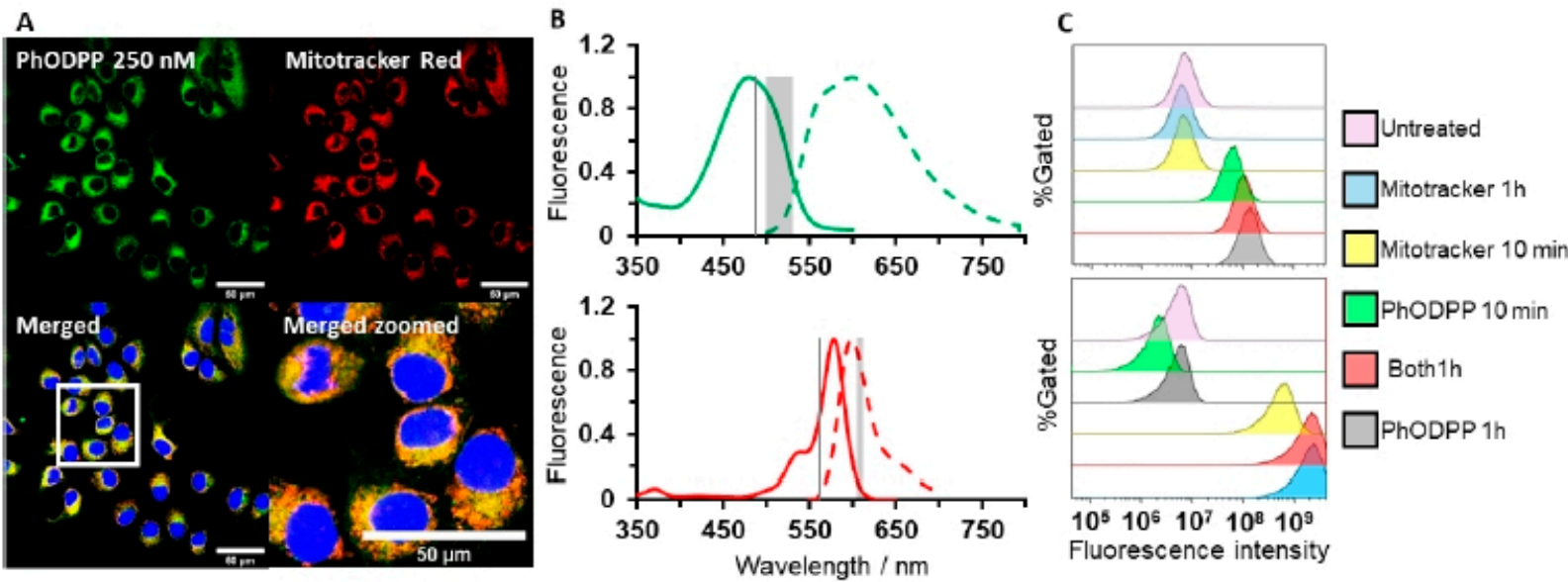

Figure 18. Top left, solid and dashed lines illustrate the absorption and TPA spectral changes respectively of (a) 3a gray with 4a black molecules in DMSO; (b) 4b appeared black in DMSO but gray in water, (c) while 4c showed black and gray colors in DMSO and water, accordingly. Copyright, 2015, reproduced with permission from Reference [41]. Top Right, Multiple-photon confocal fluorescence images displaying stained mitochondria of HeLa live cancerous cervical cells incubated for an hour using (a) $1 \mathrm{~mm}$ coloring agent of $4 \mathrm{~b}$ along with (b) MitoTracker Green $250 \mathrm{~nm}$. (c) Combined images demonstrating 4b molecule's co-positioning with the mitochondria. Copyright, 2015, reproduced with permission from Reference [41]. Bottom, (A) MCF7 intracellularly distributed PhODPP at (250 nm, $0.163 \mathrm{mg} \mathrm{mL}^{-1}$ ) with MitoTracker Red at $\left(250 \mathrm{~nm}, 0.133 \mathrm{mg} \mathrm{mL}^{-1}\right)$. (B) Dashed and solid lines illustrates the PhODPP normalized absorption and fluorescence emission spectral changes (top spectrum) with a MitoTracker Red (bottom spectrum) in water (0.2\% DMSO). Solid grey lines represents PhODPP and MitoTracker Red excited wavelengths of 488 and $561 \mathrm{~nm}$ correspondingly, while their spectral emissive regions (grey bands) are 500-526 nm (PhODPP) and 614-620 nm (MitoTracker Red) accordingly. (C) MCF7 cells flow cytometric histogram treated using PhODPP and MitoTracker Red at $(250 \mathrm{~nm})$ concentrations under the same spectroscopic condition (similar excitation and emission wavelengths). Copyright, 2020 reproduced with permission from Reference [15].

Regardless of such observed positive results, these kinds of probes pose harmful effects to the mitochondrial potential from their lipophilic cationic nature; as such, the effect consequently disrupts their critical working potential, thereby decreasing their effectiveness to function well as a staining agent. To improve this situation, very recently, Abelha et al. designed two non-charged neural DPP-based FPs with high selectivity and sensitivity for bio-imaging of mitochondria (second-row image $18 \mathrm{~A}-\mathrm{C})^{15}$. These probes are PhDPP bearing phosphine and PhODPP for phosphine oxide [15]. Among them, PhODPP incorporated with triphenylphosphine oxide showed greater Stoke's shift $\left(4096 \mathrm{~cm}^{-1}\right)$, with high FQY (56\%) in water (see Table 2 above), which minimizes the possibility to self-quench, thus making it very suitable agent for bio-imaging. Additionally, in vitro studies of PhODPP show no interference will cell metabolism and that it exhibits a higher cell uptake for human MCF7 tested breast cancer cell with high sensitivity down to nanomolar level compared with its counterpart. Moreover, PhODPP serves the first neural DPP-based FP, and it is among very few to do so, regardless of the chemistries involved (a nanomolar level detection). Hence, it mitigated the intermembrane potential of mitochondria occurring when using charged molecular probes, and interestingly PhODPP displayed similar performance with the commonly and commercially available MitoTracker red (a staining agent of mitochondria).

\section{Polymeric DPP-Based Fluorescent Probes}

Wang et al. synthesized four series of DPP-based polymer FPs (PCL-DPPPCL) bearing varied chain lengths (P1 and P2) of polycaprolactones (PCLs) from DPP-OH initiator through ring-opening polymerization [77]. Optical features of these polymers were studied in relation to the DPP-OH small molecule. This study revealed P3 and P4 polymers having moderate and lengthy PCL side-chain ( $n>100)$ to have significantly stifled the $\pi-\pi$ 
interactions existing between DPPs, and this leads to a sustained bright yellow emission displayed by these DPP-based polymers in a solid-state, under UV-light irradiation, suggesting this strategy to be applicable to other organic chromophores towards aiding the typical challenges of ACQ. In addition to that, upgrading the biodegradability of PCL by using the strongly emissive DPP along with other conjugated molecular systems could widen the biomedical scope horizons like in tissue engineering and bio-imaging research fields. Nanoparticles (NPs) dispersion of these polymers in water, under an ambient condition of UV light $365 \mathrm{~nm}$, shows P3 and P4 to fluoresce strongly yellow, while P1 and P2 fluoresce to weakly pink, with DPP-OH small molecules being completely non-emissive. To further understand the aggregate emission of the DPPs with this NPs, a steady-state emission was conducted, and the NPs produced by DPP-OH were intensely quenched in relation to those produced by the polymers $\mathrm{P} 1-\mathrm{P} 4$; this is in good agreement with their displayed phenomena under UV light.

Amidst the incorporation of DPP with furan donor to give FDPP derivatives as presented in (Scheme 2C) $)^{52}$ above, Oklem et al. established a subsequent polymerization of FDPP with thiophene and produces PDPP2FT a NIR-polymer $789 \mathrm{~nm}$ with PCE of $5 \%$ when used as the active material in organic solar cells (OSCs). Accordingly, replacing the thiophene donor with selenophene donor unit bathochromically shifts the synthesized material's absorption more to the NIR region. The replacement of thiophene with the selenium affords a novel NIR-absorbing polymer PFDPPSe with pronounced red-shift absorption of $832 \mathrm{~nm}$ (Figure 19a) ${ }^{52}$ [52]. One of the very rarely observed DPP-based polymers with no thiophene scaffold on its molecular building block. This material's optical energy band gap was found to be below $1.34 \mathrm{eV}$, which is determined from the onset absorption peak 930 $\mathrm{nm}$ with HOMO/LUMO values of 5.46/3.81 eV correspondingly (Figure 19c) ${ }^{52}$, consistent to those of PDPP2FT analogue. The selenium bigger size in relation to sulfur leads to lowering of selenophene's aromaticity as a result of poor selenium orbitals overlap with the carbon-building-block $\pi$-system. This undoubtedly leads to a value-added molecular energy levels and thus the more observed red-shifted in the absorption profile of the PFDPPSe DPP-based polymer. These data revealed the effectiveness of selenophene motifs in advancing an extreme NIR-absorbing polymer for OSCs application.

Porous organic polymer (POP) is a promising fluorescent sensing platform in reversible heterogeneous systems, due to simplicity in optimizing it chemical and nanoporous structure, and insolubility. Following these findings, in same year (2018), authors of Reference [78] reported another DPP-based polymer FP, incorporating tetrakis(4ethynylphenyl)methane via Sonogoshira coupling reaction (Uni-DPP-PPN) purposely to afford bulk material with potential to escape forming aggregated fluorophores and energetic positions to act towards an analyte (Figure 19 scheme) ${ }^{78}$. This synthesized DPP derivative showed protected penetrability that is of vital importance for sensing chemicals. First, it hampered the ACQ with red-shift emission characteristics of the parent monomer. Second, a guest-host interaction was facilitated as it shows ability of accommodating small molecules efficiently with enhanced sensitivity towards fluoride ion down to $18 \mathrm{ppb}$ detection limit with a very high selectivity for the fluoride as several anionic solutions were tested, but the material was found unreactive, except with the $\mathrm{F}^{-}$solution due to its high nucleophilicity (third row, Figure 19e ${ }^{78}$. Moreover, the material is highly reproducible while washing it with dilute acid, thus inferring its superiority for sensing fluoride ions. The material displayed a broad absorption from 200 to $600 \mathrm{~nm}$, peaking at $460 \mathrm{~nm}$, and its red emission was observed in solid-state at $684 \mathrm{~nm}$ after excited at $460 \mathrm{~nm}$ with an observed FQY of $12.6 \%$; this is attributed to the Uni-DPP-PN porosity network, with its optimal distribution of the DPP motifs; thus, it deterred the ACQ, and as a result, it enhanced its solid-state fluorescence efficiency (third row, Figure 19b $)^{78}$.

And very recently, a series of conjugated low-energy-band gap polythiophene (PT) polymers (PDPP, PDPCz21 and PDPCz11) composed of 2-ethylhexyl-optimized diketopyrrolopyrrole (TDPP) moieties (electron-acceptor component) plus substituted-terpyridine carbazole (TPCz) moieties (electron-donor component) (Scheme 2B) ${ }^{79}$ above were reported 
by Wang et al. for application in photovoltaic devices (PVDs) [79]. The energy band gaps of these polymers increased with the increase TPC $z$ content units. These materials were blended with $\mathrm{PC}_{71} \mathrm{BM}$ for PVD and it was found that PDPP/PC ${ }_{71} \mathrm{BM}$ forms poor film quality, thus implying high degree $\pi-\pi$ stacking of TDPP in the polymer building block, this is in good agreement with the relatively lower solubility of PDPP. However, $\mathrm{PC}_{71} \mathrm{BM}$ units in the polymers shows uniform distribution in composite films of PDPCz21/PC 71 BM and PDPCz11/PC 71 BM ranging from 375 to $860 \mathrm{~nm}$ with peak absorption at $670 \mathrm{~nm}$ (Figure 19a-c, fourth row) ${ }^{79}$, and exhibited good film quality, thus indicating an improved compatibility between PT with $\mathrm{PC}_{71} \mathrm{BM}$ after successful introduction of TPC $z$ units in the molecular building block of the polymers. Therefore, bulk pendant groups like TPCz improved the permitted ability of the PT backbone and so, boosted the suitability of PT and $\mathrm{PC}_{71} \mathrm{BM}$ components.
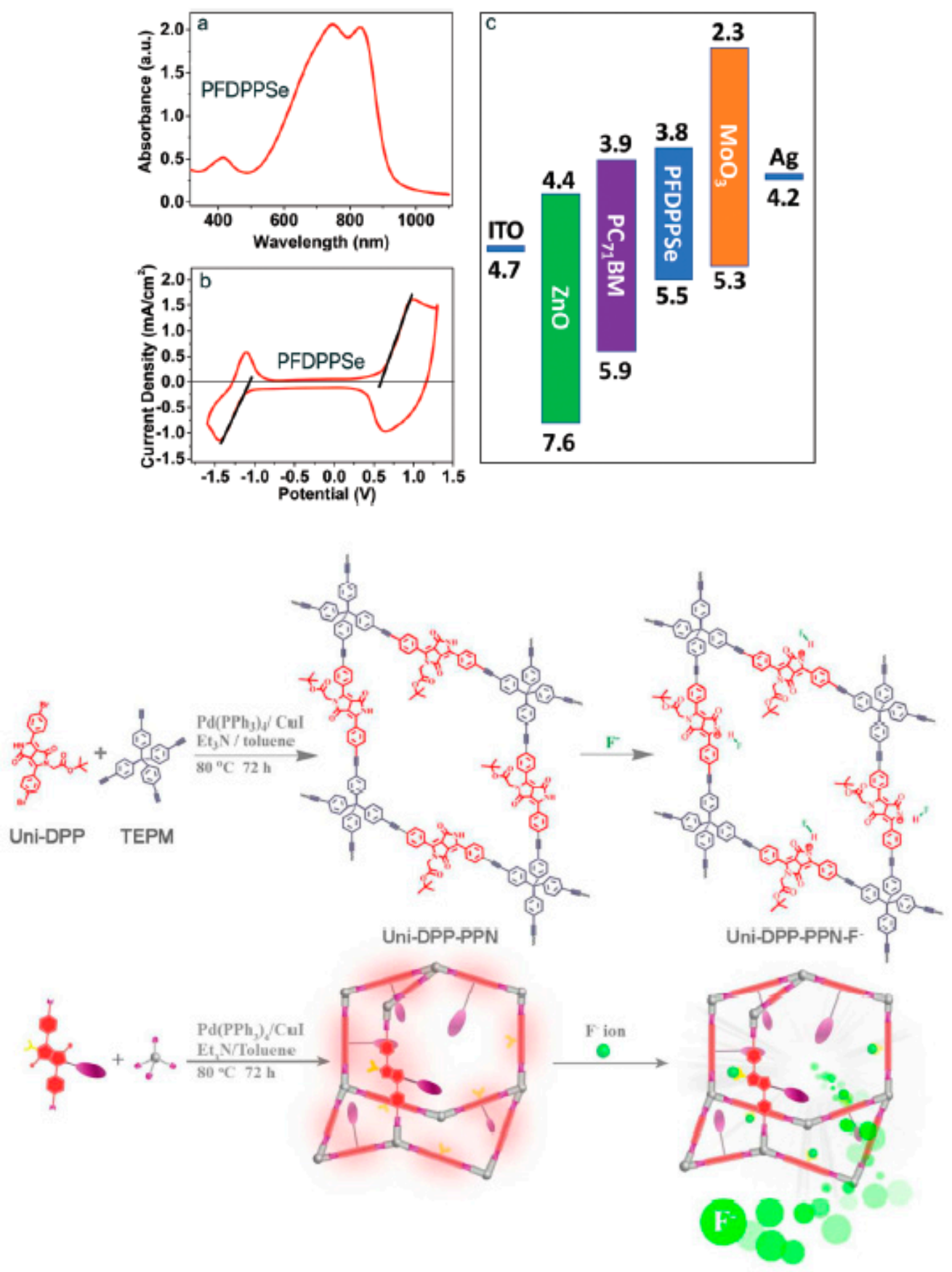

Figure 19. Cont. 

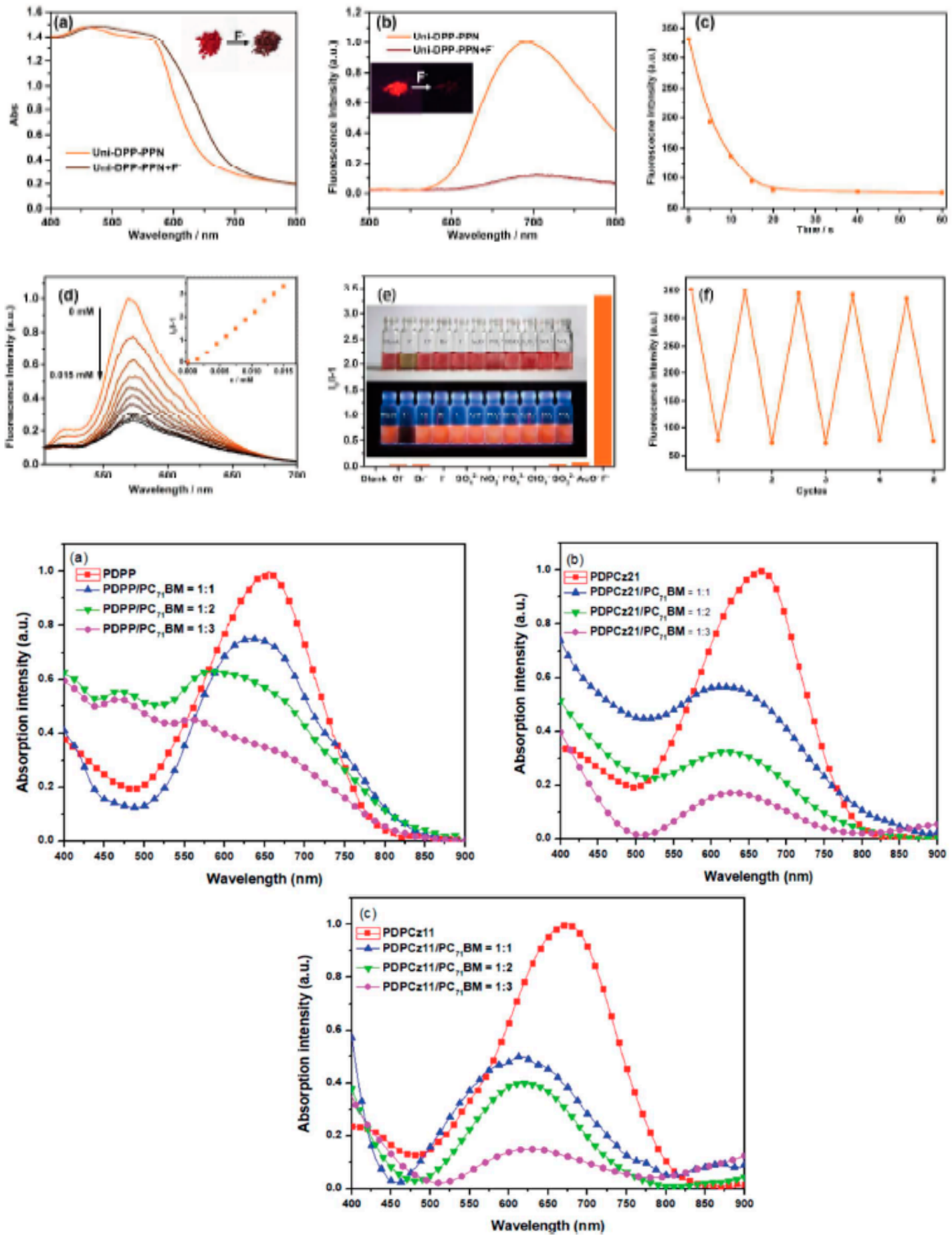

Figure 19. First row: (a) thin-film absorption spectrum (b) CV of FDDPPSe, and (c) PFDPPSe band configuration in proposed solar cell. Copyright, 2018 reproduced with permission from Reference [52]. Second row: synthetic route of Uni-DPP-PPN with its $\mathrm{F}^{-1}$ anions detection mechanism. Third row: (a) Diffuse reflectance UV-Vis spectrum of free (Uni-DPP-PPN) and bound (Uni-DPP-PPN-F $\left.{ }^{-1}\right)(\mathbf{b})$ fluorescence in solid-state for freely unbound Uni-DPP-PPN and then of bound Uni-DPP-PPN-F- (c) Detection kinetics with Uni-DPP-PPN at $0.015 \mathrm{mM} \mathrm{F}^{-1}$ concentration. (d) Uni-DPP-PPN $(0.1 \mathrm{mg} / \mathrm{mL}$ in THF) fluorescence titration using TBAF solution (e) selective recognition of fluoride anion with other interfering anions of known concentration (photographs of Uni-DPP-PPN suspended solution in THF $(0.1 \mathrm{mg} / \mathrm{mL}$ in THF) after addition of $\mathrm{F}^{-1}(0.015 \mathrm{mM})$ with other analytes $(1.5 \mathrm{mM})(\mathbf{f})$ Recognition of fluoride anion via cycling testing by Uni-DPP-PPN. Copyright, 2018 reproduced with permission from Reference [78]. Fourth row: absorption spectra of (DPP-based PT polymers): (a) PDPP/PC71BM, (b) PDPCz21/PC71BM and (c) PDPCz11/PC71BM blend thin-films. Copyright, 2020 reproduced with permission from Reference [79]. 


\section{Research Gaps, Status and Future Prospects}

From our perspective, it is imperative to note that the following key research areas need special attention, as other hidden potentials for this class of glowing DPPs could be explored in order to widen the scope:

1. DPPs' high planarity, rigidity, extended conjugation, and highly electronic as well thermally stable nature with high photostability are reasons why a lot of efforts are being devoted to utilizing such fluorochromes more than others. As mostly used DPPs are thiophene-based incorporated, at this juncture, it is also crucial to extend more on exploring other fluorescent DPPs from other incorporated donors onto the DPP acceptor component like the furan-based, pyridine-based, selenophene-based and others. This is due to observed positivity outcomes of utilizing such donor groups with DPP, as presented here, in our review of the literature, like high FQYs, the ability to make fluorescent polymers, etc. Thus, exploring newly innovative glowing DPP materials by considering other donor units is worthy to scrutinize. Precisely, selenophene-based DPPs were reported to have possessed very good optoelectronic properties like, good FQY from their (low-energy) optimized fluorophores, low energy bandgap attributed by their low-lying HOMO/LUMO along with their observed good surface morphology which resulted from the effective heavier nature of Selenium atom that favors ISC. All these are required features useful especially for applications in LOFETs, OLEDs, Solar cells and other light emitting devices.

2. Fluorescent DPPs have shown auspiciously to serve as new class of annihilators due to their appreciable stability in ambient conditions compared to ascene and rubrene, thus serving as an avenue to explore innovatively new DPP-based annihilators considering their positive outcomes (high stability), as reported herein.

3. The ability of fluorescent DPPs to form aggregates in solution (AIE) and fluoresce strongly as thin-film (solid-sate) is highly significant, as this will significantly address the ACQ issues especially for LEOFTs, OLEDs, PVDs, etc., as such applications need organic fluorophores to be compacted into solid thin-films.

4. As triphenylamine (TPA) shows very good influence on the photophysical effects of DPPs like quenched in fluorescence, it is highly commendable to note this; therefore, appropriate incorporation of TPA with DPP fluorophore is worth noting, depending on the proposed application.

5. Fluorescent DPPs prove highly promising for biomedical applications like for biomolecules detection; bio-imaging; staining of important biological components like mitochondria; detection of analytes in solution and solid-state (thin-films); and cancer therapy, like in PDT/PTT via the highly effective 2PFM with nanoparticles of DPP, with nearly zero or negligible cytotoxicity. These findings still further strengthened their useful supramolecular chemistry potentiality, hence paving a smoother way for further in-depth studies in order to widen such admirable properties more deeply and intensively in biological systems.

6. As porous fluorescent DPP polymers were reported for fluoride ion detection, such porosity could be utilized by extending it applicability to bio-imaging, in that the polymeric and porosity nature of such fluorescent materials to have significantly enhance deeper tissue penetrability by their highly conjugated system.

7. Lastly, investigating "Phosphorescence" in luminescent DPPs is highly suggestable, as no data of such phenomena exhibited by DPP-based glowing materials, to the best of our knowledge, have so far been reported, despite the high electronic, thermal and photochemical stability of DPP. Thus, scrutinizing such photophysical property would be very essential.

\section{Conclusions}

Certainly, this review article provides some insights for researchers working on DPP FPs, the versatile scope they entail and with their possible biological applications, precisely the supramolecular chemistry (SC) applications of various DPP-based FPs. This 
article accounts the scholarly contribution on the progress that has so far been made very recently, mainly within the last decade, and selectively focused discussions more from 2015 to the present by various research groups on luminescent DPPs (exceptional property). Consequently, this review of works from the literature expresses some valuable insights to researchers working on exploring novel fluorescent DPPs, with the hope to widen the research horizon. It is anticipated to mention that DPP FPs have shown promising SC potentials (deep tissue penetration, negligible cytotoxicity and cancer/tumor cells destruction) in the biological system, with hope that, in the near future, fluorescent DPPs will further boost the research dimension in this hot and intense research area of biosensors with potent new discoveries.

Author Contributions: M.A.A., writing-original draft and investigation. S.C., writing-review and editing, project administration, supervision and funding acquisition. All authors have read and agreed to the published version of the manuscript.

Funding: This work was financially supported by the Natural Science Foundation of China (Grant Nos. 21705116, 51733004, 91433115, 51633006, 51703160, 21661132006 and 21473222).

Institutional Review Board Statement: Human or Animal experiments is not conducted in this review article.

Informed Consent Statement: The paper does not contain patient experimental analysis, only a literature study.

Data Availability Statement: There is no any supplementary material attached to this article, only reviewed literature.

Conflicts of Interest: There are no conflict to declare.

\section{References}

1. Dai, J.; Ma, C.; Zhang, P.; Fu, Y.; Shen, B. Recent progress in the development of fluorescent probes for detection of biothiols. Dye. Pigment. 2020, 177, 108321. [CrossRef]

2. Jiang, X.; Wang, L.; Tang, H.; Cao, D.; Chen, W. Diketopyrrolopyrrole: An emerging phototherapy agent in fighting cancer. Dye. Pigment. 2020, 181, 108599. [CrossRef]

3. Li, W.; Wang, L.; Tang, H.; Cao, D. Diketopyrrolopyrrole-based fluorescent probes for detection and bioimaging: Current progresses and perspectives. Dye. Pigment. 2019, 162, 934-950. [CrossRef]

4. Li, J.; Pu, K. Development of organic semiconducting materials for deep-tissue optical imaging, phototherapy and photoactivation. Chem. Soc. Rev. 2019, 48, 38-71. [CrossRef] [PubMed]

5. Chakali, M.; Mandal, H.; Venkatesan, M.; Dyaga, B.; Rao, V.J.; Bangal, P.R. Charge Separation and Singlet Fission in Covalently Linked Diketopyrrolopyrrole Derivatives and Triphenylamine Triad in Solution. J. Photochem. Photobiol. A Chem. 2020, 406, 113017. [CrossRef]

6. Zhou, K.; Dai, K.; Liu, C.; Shen, C. Flexible conductive polymer composites for smart wearable strain sensors. SmartMat 2020, 1, e1010. [CrossRef]

7. Antón-García, D.; Warnan, J.; Reisner, E. A diketopyrrolopyrrole dye-based dyad on a porous $\mathrm{TiO}_{2}$ photoanode for solar-driven water oxidation. Chem. Sci. 2020, 11, 12769-12776. [CrossRef]

8. Hwang, T.G.; Kim, G.-Y.; Han, J.-I.; Kim, S.; Kim, J.P. Enhancement of Lipid Productivity of Chlorella sp. Using Light-Converting Red Fluorescent Films Based on Aggregation-Induced Emission. ACS Sustain. Chem. Eng. 2020, 8, 15888-15897. [CrossRef]

9. Liu, Q.; Bottle, S.E.; Sonar, P. Developments of Diketopyrrolopyrrole-Dye-Based Organic Semiconductors for a Wide Range of Applications in Electronics. Adv. Mater. 2020, 32, 1903882. [CrossRef]

10. Zhang, R.; Sun, M.; Wang, X.; Yan, H.; Zhang, G.; Zhang, Q. Polymerizations of Diketopyrrolopyrrole-Type Dyes in Unconventional Orientation. ACS Appl. Polym. Mater. 2020, 2, 5698-5704. [CrossRef]

11. Wang, Y.; Yang, J.; Gong, Y.; Fang, M.; Li, Z.; Tang, B.Z. Host-guest materials with room temperature phosphorescence: Tunable emission color and thermal printing patterns. SmartMat 2020, 1, e1006. [CrossRef]

12. Wang, Q.; Xia, B.; Xu, J.; Niu, X.; Cai, J.; Shen, Q.; Wang, W.; Huang, W.; Fan, Q. Biocompatible small organic molecule phototheranostics for NIR-II fluorescence/photoacoustic imaging and simultaneous photodynamic/photothermal combination therapy. Mater. Chem. Front. 2019, 3, 650-655. [CrossRef]

13. Ghosh, S.; Shankar, S.; Philips, D.S.; Ajayaghosh, A. Diketopyrrolopyrrole-based functional supramolecular polymers: Nextgeneration materials for optoelectronic applications. Mater. Today Chem. 2020, 16, 100242. [CrossRef] 
14. Chiminazzo, A.; Borsato, G.; Favero, A.; Fabbro, C.; McKenna, C.E.; Dalle Carbonare, L.G.; Valenti, M.T.; Fabris, F.; Scarso, A. Diketopyrrolopyrrole Bis-Phosphonate Conjugate: A New Fluorescent Probe for In Vitro Bone Imaging. Chem. A Eur. J. 2019, 25, 3617-3626. [CrossRef]

15. Abelha, T.F.; Morris, G.; Lima, S.M.; Andrade, L.H.; McLean, A.J.; Alexander, C.; Calvo-Castro, J.; McHugh, C.J. Development of a Neutral Diketopyrrolopyrrole Phosphine Oxide for the Selective Bioimaging of Mitochondria at the Nanomolar Level. Chem. A Eur. J. 2020. [CrossRef] [PubMed]

16. Kaur, M.; Choi, D.H. Diketopyrrolopyrrole: Brilliant red pigment dye-based fluorescent probes and their applications. Chem. Soc. Rev. 2015, 44, 58-77. [CrossRef]

17. Sun, X.-Y.; Liu, T.; Sun, J.; Wang, X.-J. Synthesis and application of coumarin fluorescence probes. Rsc Adv. 2020, 10, 10826-10847. [CrossRef]

18. Rajasekar, M. Recent development in fluorescein derivatives. J. Mol. Struct. 2020, 1224, 129085. [CrossRef]

19. Fu, Z.-H.; Han, X.; Shao, Y.; Fang, J.; Zhang, Z.-H.; Wang, Y.-W.; Peng, Y. Fluorescein-based chromogenic and ratiometric fluorescence probe for highly selective detection of cysteine and its application in bioimaging. Anal. Chem. 2017, 89, 1937-1944. [CrossRef] [PubMed]

20. Zhang, Q.; Wong, K.M.-C. Photophysical, ion-sensing and biological properties of rhodamine-containing transition metal complexes. Coord. Chem. Rev. 2020, 416, 213336. [CrossRef]

21. Fischer, G.M.; Daltrozzo, E.; Zumbusch, A. Selective NIR chromophores: Bis (pyrrolopyrrole) cyanines. Angew. Chem. Int. Ed. 2011, 50, 1406-1409. [CrossRef]

22. Fischer, G.M.; Isomäki-Krondahl, M.; Göttker-Schnetmann, I.; Daltrozzo, E.; Zumbusch, A. Pyrrolopyrrole cyanine dyes: A new class of near-infrared dyes and fluorophores. Chem. A Eur. J. 2009, 15, 4857-4864. [CrossRef]

23. Wiktorowski, S.; Rosazza, C.; Winterhalder, M.J.; Daltrozzo, E.; Zumbusch, A. Water-soluble pyrrolopyrrole cyanine (PPCy) NIR fluorophores. Chem. Commun. 2014, 50, 4755-4758. [CrossRef]

24. Sun, C.; Du, W.; Wang, B.; Dong, B.; Wang, B. Research progress of near-infrared fluorescence probes based on indole heptamethine cyanine dyes in vivo and in vitro. Bmc Chem. 2020, 14, 1-28. [CrossRef]

25. Li, Y.; Zhou, Y.; Yue, X.; Dai, Z. Cyanine Conjugate-Based Biomedical Imaging Probes. Adv. Healthc. Mater. 2020, 9, 2001327. [CrossRef] [PubMed]

26. Shimizu, S.; Iino, T.; Araki, Y.; Kobayashi, N. Pyrrolopyrrole aza-BODIPY analogues: A facile synthesis and intense fluorescence. Chem. Commun. 2013, 49,1621-1623. [CrossRef]

27. Alnoman, R.B.; Parveen, S.; Hagar, M.; Ahmed, H.A.; Knight, J.G. A new chiral boron-dipyrromethene (BODIPY)-based fluorescent probe: Molecular docking, DFT, antibacterial and antioxidant approaches. J. Biomol. Struct. Dyn. 2020, 38, 5429-5442. [CrossRef]

28. Wang, J.; Xu, W.; Yang, Z.; Yan, Y.; Xie, X.; Qu, N.; Wang, Y.; Wang, C.; Hua, J. New diketopyrrolopyrrole-based ratiometric fluorescent probe for intracellular esterase detection and discrimination of live and dead cells in different fluorescence channels. Acs Appl. Mater. Interfaces 2018, 10, 31088-31095. [CrossRef] [PubMed]

29. Du, W.; Ohayon, D.; Combe, C.; Mottier, L.; Maria, I.P.; Ashraf, R.S.; Fiumelli, H.; Inal, S.; McCulloch, I. Improving the compatibility of diketopyrrolopyrrole semiconducting polymers for biological interfacing by lysine attachment. Chem. Mater. 2018, 30, 6164-6172. [CrossRef]

30. Ni, Z.; Dong, H.; Wang, H.; Ding, S.; Zou, Y.; Zhao, Q.; Zhen, Y.; Liu, F.; Jiang, L.; Hu, W. Quinoline-Flanked Diketopyrrolopyrrole Copolymers Breaking through Electron Mobility over $6 \mathrm{~cm}^{2} \mathrm{~V}^{-1} \mathrm{~s}^{-1}$ in Flexible Thin Film Devices. Adv. Mater. 2018, 30, 1704843. [CrossRef] [PubMed]

31. Wang, Z.; Liu, Z.; Ning, L.; Xiao, M.; Yi, Y.; Cai, Z.; Sadhanala, A.; Zhang, G.; Chen, W.; Sirringhaus, H. Charge mobility enhancement for conjugated DPP-selenophene polymer by simply replacing one bulky branching alkyl chain with linear one at each DPP unit. Chem. Mater. 2018, 30, 3090-3100. [CrossRef]

32. Chandran, D.; Lee, K.-S. Diketopyrrolopyrrole: A versatile building block for organic photovoltaic materials. Macromol. Res. 2013, 21, 272-283. [CrossRef]

33. Patil, H.; Gupta, A.; Bilic, A.; Bhosale, S.V.; Bhosale, S.V. A solution-processable electron acceptor based on diketopyrrolopyrrole and naphthalenediimide motifs for organic solar cells. Tetrahedron Lett. 2014, 55, 4430-4432. [CrossRef]

34. Patil, Y.; Misra, R. Rational molecular design towards NIR absorption: Efficient diketopyrrolopyrrole derivatives for organic solar cells and photothermal therapy. J. Mater. Chem. C 2019, 7, 13020-13031. [CrossRef]

35. Yang, X.; Cui, Y.; Li, Y.; Zheng, L.; Xie, L.; Ning, R.; Liu, Z.; Lu, J.; Zhang, G.; Liu, C. A new diketopyrrolopyrrole-based probe for sensitive and selective detection of sulfite in aqueous solution. Spectrochim. Acta Part A Mol. Biomol. Spectrosc. 2015, 137, 1055-1060. [CrossRef]

36. Schutting, S.; Borisov, S.M.; Klimant, I. Diketo-pyrrolo-pyrrole dyes as new colorimetric and fluorescent pH indicators for optical carbon dioxide sensors. Anal. Chem. 2013, 85, 3271-3279. [CrossRef]

37. Kaur, M.; Choi, D.H. Dual channel receptor based on diketopyrrolopyrrole alkyne conjugate for detection of $\mathrm{Hg}^{2+} / \mathrm{Cu}^{2+}$ by "naked eye" and fluorescence. Sens. Actuators B Chem. 2014, 190, 542-548. [CrossRef]

38. Aigner, D.; Ungerböck, B.; Mayr, T.; Saf, R.; Klimant, I.; Borisov, S.M. Fluorescent materials for pH sensing and imaging based on novel 1, 4-diketopyrrolo-[3, 4-c] pyrrole dyes. J. Mater. Chem. C 2013, 1, 5685-5693. [CrossRef] 
39. Fischer, G.M.; Ehlers, A.P.; Zumbusch, A.; Daltrozzo, E. Near-infrared dyes and fluorophores based on diketopyrrolopyrroles. Angew. Chem. Int. Ed. 2007, 46, 3750-3753. [CrossRef]

40. Qu, Y.; Wu, Y.; Gao, Y.; Qu, S.; Yang, L.; Hua, J. Diketopyrrolopyrrole-based fluorescent conjugated polymer for application of sensing fluoride ion and bioimaging. Sens. Actuators B Chem. 2014, 197, 13-19. [CrossRef]

41. Grzybowski, M.; Glodkowska-Mrowka, E.; Hugues, V.; Brutkowski, W.; Blanchard-Desce, M.; Gryko, D.T. Polar DiketopyrrolopyrroleImidazolium Salts as Selective Probes for Staining Mitochondria in Two-Photon Fluorescence Microscopy. Chem. A Eur. J. 2015, 21, 9101-9110. [CrossRef]

42. Tang, S.; Zadeh, E.H.G.; Kim, B.; Toomey, N.T.; Bondar, M.V.; Belfield, K.D. Protein-induced fluorescence enhancement of two-photon excitable water-soluble diketopyrrolopyrroles. Org. Biomol. Chem. 2017, 15, 6511-6519. [CrossRef]

43. Ftouni, H.; Bolze, F.D.R.; de Rocquigny, H.; Nicoud, J.-F.O. Functionalized two-photon absorbing diketopyrrolopyrrole-based fluorophores for living cells fluorescent microscopy. Bioconjugate Chem. 2013, 24, 942-950. [CrossRef]

44. Ren, X.; Yang, F.; Gao, X.; Cheng, S.; Zhang, X.; Dong, H.; Hu, W. Organic Field-Effect Transistor for Energy-Related Applications: Low-Power-Consumption Devices, Near-Infrared Phototransistors, and Organic Thermoelectric Devices. Adv. Energy Mater. 2018, 8, 1801003. [CrossRef]

45. Yang, S.F.; Liu, Z.T.; Cai, Z.X.; Dyson, M.J.; Stingelin, N.; Chen, W.; Ju, H.J.; Zhang, G.X.; Zhang, D.Q. Diketopyrrolopyrrole-Based Conjugated Polymer Entailing Triethylene Glycols as Side Chains with High Thin-Film Charge Mobility without Post-Treatments. Adv. Sci. 2017, 4, 1700048. [CrossRef]

46. Yang, S.F.; Zhang, X.; Chen, P.L.; Liu, Z.T.; Tian, J.W.; Zhang, G.X.; Zhang, D.Q. Diketopyrrolopyrrole-Based Semiconducting Polymer with Both Hydrophobic Alkyl and Hydrophilic Tetraethylene Glycol Chains for Monolayer Transistor and Sensing Application. Adv. Electron. Mater. 2017, 3, 1700120. [CrossRef]

47. Yang, Y.; Liu, Z.; Chen, L.; Yao, J.; Lin, G.; Zhang, X.; Zhang, G.; Zhang, D. Conjugated Semiconducting Polymer with Thymine Groups in the Side Chains: Charge Mobility Enhancement and Application for Selective Field-Effect Transistor Sensors toward CO and H2S. Chem. Mater. 2019, 31, 1800-1807. [CrossRef]

48. Zhang, D. Significant improvement of the semiconducting performance for the DPP-quaterthiophene conjugated polymer through side-chain engineering via hydrogen-bonding. In Abstracts of Papers of the American Chemical Society; American Chemical Society: Washington, DC, USA, 2016.

49. Fu, S.; Wan, X.; Du, C.; Wang, H.; Zhou, J.; Wang, Z. A novel fluorescent probe for the early detection of prostate cancer based on endogenous zinc sensing. Prostate 2019, 79, 1406-1413. [CrossRef]

50. Pang, S.; Más-Montoya, M.; Xiao, M.; Duan, C.; Wang, Z.; Liu, X.; Janssen, R.A.; Yu, G.; Huang, F.; Cao, Y. Adjusting Aggregation Modes and Photophysical and Photovoltaic Properties of Diketopyrrolopyrrole-Based Small Molecules by Introducing B $\leftarrow$ N Bonds. Chem. A Eur. J. 2019, 25, 564-572. [CrossRef]

51. Jin, Y.; Xu, Y.; Liu, Y.; Wang, L.; Jiang, H.; Li, X.; Cao, D. Synthesis of novel diketopyrrolopyrrole-based luminophores showing crystallization-induced emission enhancement properties. Dye. Pigment. 2011, 90, 311-318. [CrossRef]

52. Oklem, G.; Song, X.; Toppare, L.; Baran, D.; Gunbas, G. A new NIR absorbing DPP-based polymer for thick organic solar cells. J. Mater. Chem. C 2018, 6, 2957-2961. [CrossRef]

53. Patil, Y.; Misra, R. Metal Functionalized Diketopyrrolopyrroles: A Promising Class of Materials for Optoelectronic Applications. Chem. Rec. 2020, 20, 596-603. [CrossRef]

54. Langhals, H.; Limmert, M.; Lorenz, I.P.; Mayer, P.; Piotrowski, H.; Polborn, K. Chromophores encapsulated in gold complexes: DPP dyes with novel properties. Eur. J. Inorg. Chem. 2000, 2000, 2345-2349. [CrossRef]

55. Lorenz, I.P.; Limmert, M.; Mayer, P.; Piotrowski, H.; Langhals, H.; Poppe, M.; Polborn, K. DPP Dyes as Ligands in Transition-Metal Complexes. Chem. A Eur. J. 2002, 8, 4047-4055. [CrossRef]

56. Bürckstümmer, H.; Weissenstein, A.; Bialas, D.; Würthner, F. Synthesis and characterization of optical and redox properties of bithiophene-functionalized diketopyrrolopyrrole chromophores. J. Org. Chem. 2011, 76, 2426-2432. [CrossRef]

57. Dhar, J.; Venkatramaiah, N.; Anitha, A.; Patil, S. Photophysical, electrochemical and solid state properties of diketopyrrolopyrrole based molecular materials: Importance of the donor group. J. Mater. Chem. C 2014, 2, 3457-3466. [CrossRef]

58. Wang, K.; Feng, J.; Xu, J.; Li, J.; Mai, M.; Wang, X.; Wang, L. Engineering aromatic heterocycle strategy: Improving copper electrodeposition performance via tuning the bandgap of diketopyrrolopyrrole-based leveler. Tetrahedron 2020, 76, 130882. [CrossRef]

59. Heyer, E.; Lory, P.; Leprince, J.; Moreau, M.; Romieu, A.; Guardigli, M.; Roda, A.; Ziessel, R. Highly Fluorescent and Water-Soluble Diketopyrrolopyrrole Dyes for Bioconjugation. Angew. Chem. 2015, 127, 3038-3042. [CrossRef]

60. Hwang, T.G.; Han, G.R.; Lee, J.M.; Lee, J.W.; Kim, H.M.; Hwang, D.; Kim, S.K.; Kim, J.P. Fluorescence Quenching of 4, 4'Dimethoxytriphenylamine-Substituted Diketopyrrolopyrrole via Intramolecular Photoinduced Electron Transfer. J. Phys. Chem. C 2019, 123, 24263-24274. [CrossRef]

61. Hwang, T.G.; Kim, J.Y.; Namgoong, J.W.; Lee, J.M.; Yuk, S.B.; Kim, S.H.; Kim, J.P. Aggregation induced emission of diketopyrrolopyrrole (DPP) derivatives for highly fluorescent red films. Photochem. Photobiol. Sci. 2019, 18, 1064-1074. [CrossRef]

62. Cai, Y.; Liang, P.; Tang, Q.; Yang, X.; Si, W.; Huang, W.; Zhang, Q.; Dong, X. Diketopyrrolopyrrole-triphenylamine organic nanoparticles as multifunctional reagents for photoacoustic imaging-guided photodynamic/photothermal synergistic tumor therapy. Acs Nano 2017, 11, 1054-1063. [CrossRef] 
63. Shen, L.; Tang, Z.; Wang, X.; Liu, H.; Chen, Y.; Li, X. Effects of aromatic substituents on the electronic structure and excited state energy levels of diketopyrrolopyrrole derivatives for singlet fission. Phys. Chem. Chem. Phys. 2018, 20, 22997-23006. [CrossRef]

64. Pun, A.B.; Campos, L.M.; Congreve, D.N. Tunable Emission from Triplet Fusion Upconversion in Diketopyrrolopyrroles. J. Am. Chem. Soc. 2019, 141, 3777-3781. [CrossRef] [PubMed]

65. Yao, Y.; Chen, Y.; Wang, H.; Samorì, P. Organic photodetectors based on supramolecular nanostructures. SmartMat 2020, 1. [CrossRef]

66. Graziano, G. Solid-state fluorescence: Under pressure. Nat. Rev. Chem. 2017, 1. [CrossRef]

67. Kuwabara, J.; Yamagata, T.; Kanbara, T. Solid-state structure and optical properties of highly fluorescent diketopyrrolopyrrole derivatives synthesized by cross-coupling reaction. Tetrahedron 2010, 66, 3736-3741. [CrossRef]

68. Turkcen, S.; Dincalp, H.; Saltan, G.M. Synthesis and determination of fluorescence properties of new soluble diketopyrrolopyrrole type photosensitizers. J. Mol. Struct. 2019, 1195, 485-493. [CrossRef]

69. Tao, T.; Zhao, J.; Fan, B.; Chen, Z.; Sun, J. Terminal modulation of asymmetrical D- A - D- $\pi$ furan-containing diketopyrrolopyrrole chromophores for intramolecular charge transfer properties. Dye. Pigment. 2020, 177, 108277. [CrossRef]

70. Shi, C.Y.; Zhang, Q.; Tian, H.; Qu, D.H. Supramolecular adhesive materials from small-molecule self-assembly. SmartMat 2020, 1, e1012. [CrossRef]

71. Mei, J.; Graham, K.R.; Stalder, R.; Tiwari, S.P.; Cheun, H.; Shim, J.; Yoshio, M.; Nuckolls, C.; Kippelen, B.; Castellano, R.K. Self-assembled amphiphilic diketopyrrolopyrrole-based oligothiophenes for field-effect transistors and solar cells. Chem. Mater. 2011, 23, 2285-2288. [CrossRef]

72. Ghosh, S.S.; Serrano, L.A.; Ebenhoch, B.; Rotello, V.M.; Cooke, G.; Samuel, I.D. Organic solar cells based on acceptor-functionalized diketopyrrolopyrrole derivatives. J. Photonics Energy 2015, 5, 057215. [CrossRef]

73. Brimble, M.A.; Rani, A.; Kavianinia, I.; Hume, P.; Rodriguez, L.M.M.D.L.; Kihara, S.; Williams, D.E.; McGillivray, D.J.; Plank, N.; Gerrard, J.A. Directed Self-Assembly of Peptide-Diketopyrrolopyrrole Conjugates-a Platform for Bio-Organic Thin Film Preparation. Soft Matter 2020, 16, 6563-6571.

74. Kaur, M.; Cho, M.J.; Choi, D.H. Chemodosimeter approach: Selective detection of fluoride ion using a diketopyrrolopyrrole derivative. Dye. Pigment. 2014, 103, 154-160. [CrossRef]

75. Cai, Y.; Si, W.; Huang, W.; Chen, P.; Shao, J.; Dong, X. Organic dye based nanoparticles for cancer phototheranostics. Small 2018, 14, 1704247. [CrossRef]

76. Du, C.; Fu, S.; Wang, X.; Sedgwick, A.C.; Zhen, W.; Li, M.; Li, X.; Zhou, J.; Wang, Z.; Wang, H. Diketopyrrolopyrrole-based fluorescence probes for the imaging of lysosomal $\mathrm{Zn}^{2+}$ and identification of prostate cancer in human tissue. Chem. Sci. 2019, 10, 5699-5704. [CrossRef] [PubMed]

77. Wang, K.; Luo, Y.; Huang, S.; Yang, H.; Liu, B.; Wang, M. Highly fluorescent polycaprolactones decorated with di (thiophene-2yl)-diketopyrrolopyrrole: A covalent strategy of tuning fluorescence properties in solid states. J. Polym. Sci. Part A Polym. Chem. 2015, 53, 1032-1042. [CrossRef]

78. Bi, S.; Li, Y.; Zhang, S.; Hu, J.; Wang, L.; Liu, H. A diketopyrrolopyrrole-based fluorescent porous organic polymer as fluoride sensing monolithic device. J. Mater. Chem. C 2018, 6, 3961-3967. [CrossRef]

79. Wang, S.-H.; Wang, T.-W.; Tsai, H.-C.; Yang, P.-C.; Huang, C.-F.; Lee, R.-H. Synthesis of the diketopyrrolopyrrole/terpyridine substituted carbazole derivative based polythiophenes for photovoltaic cells. Rsc Adv. 2020, 10, 9525-9535. [CrossRef] 